Documentation for DOE/SC/NP

Quadrennial Low Energy Nuclear Physics (LENP) Review

M. A. Stoyer, C. Wu, N. D. Scielzo, D. P. McNabb

September 7, 2011 
This document was prepared as an account of work sponsored by an agency of the United States government. Neither the United States government nor Lawrence Livermore National Security, LLC, nor any of their employees makes any warranty, expressed or implied, or assumes any legal liability or responsibility for the accuracy, completeness, or usefulness of any information, apparatus, product, or process disclosed, or represents that its use would not infringe privately owned rights. Reference herein to any specific commercial product, process, or service by trade name, trademark, manufacturer, or otherwise does not necessarily constitute or imply its endorsement, recommendation, or favoring by the United States government or Lawrence Livermore National Security, LLC. The views and opinions of authors expressed herein do not necessarily state or reflect those of the United States government or Lawrence Livermore National Security, LLC, and shall not be used for advertising or product endorsement purposes.

This work performed under the auspices of the U.S. Department of Energy by Lawrence Livermore National Laboratory under Contract DE-AC52-07NA27344. 


\title{
Documentation for DOE/SC/NP Quadrennial Low Energy Nuclear Physics (LENP) Review
}

\author{
August 31, 2011
}

Mark A. Stoyer, Ching-Yen Wu, Nicholas D. Scielzo, and Dennis P. McNabb

This work performed under the auspices of the U.S. Department of Energy by Lawrence Livermore National Laboratory under Contract DE-AC52-07NA27344. 


\section{Introduction to Low Energy Nuclear Physics at LLNL}

Research at LLNL is primarily directed towards satisfying its principal missions in international security, fusion energy, and US nuclear security. Towards this end, a core LLNL strategy has been to advance fundamental scientific understanding to succeed in its purpose, and to recruit and develop a skilled workforce for the future. Research conducted in the Physics Division at LLNL is primarily focused on bringing the highest quality science to bear on LLNL programs to achieve broad success in its overall mission. In this regard, the "applied-science" missions at LLNL are actually quite well-aligned with the fundamental-science research aims of the Office of Science. We aim to perform research for DOE/SC/NP at LLNL that has the greatest leverage towards answering fundamental problems in nuclear science -- both at the forefront of fundamental science and important to the nation's security. Towards this end, LLNL brings unique capabilities, resources, and skills to bear on the most scientifically challenging endeavors of the $\mathrm{DOE} / \mathrm{SC} / \mathrm{NP}$ mission and enhances the training of the nuclear science workforce in general. LLNL is a world leader in cost-effective high-performance computing, has a broad collection base for Laboratory Directed Research and Development (LDRD), and an excellent track record in solving multi-disciplinary and engineering problems.

While the Experimental Nuclear Physics (ENP) Group at LLNL is not primarily supported by the Office of Science and there is no DOE/SC/NP "research facility" located at LLNL, our laboratory has a strong history of tackling large and significant national security challenges for the US and has leveraged its unique capabilities, workforce, and resources to address scientifically important challenges that are consistent with NSAC established research priorities. Within LLNL, there is growing realization that our high overhead costs are interfering with our increasing desire for more effective collaboration with basic research communities, but these same overhead costs help support the extensive and unique capabilities that are also used in the service of fundamental research.

While a traditional DOE/SC laboratory is supported almost entirely by Office of Science funding, enabling researchers to devote nearly full-time to basic research, only the postdocs at LLNL are supported full time on basic research projects (with $25 \%$ of this support provided by the institute). Staff scientists in the ENP Group are supported mainly by other sources of funding (LDRD, NIF, NNSA, NA22, DNDO, DTRA, etc.) with staff members typically only supported $5-10 \%$ by DOE/SC/NP and the resulting $80-90 \%$ of their time spent on applied research for the (national security) programs at LLNL; very much analogous to a university research group with a PI supported $25 \%$ by DOE/SC and $75 \%$ by teaching assignments. For optimal mentoring of PDs and leverage of other resources, support for researchers at LLNL should be nearer to $20 \%$. The net result is a highly leveraged program that we believe substantially benefits both DOE/SC/NP and LLNL, a symbiotic relationship yielding a highquality workforce performing high-quality science with unique laboratory capabilities. 
U.S, DEPARTKENT OF ENERGY FIELD WORK PROPOSAL - FY 2013

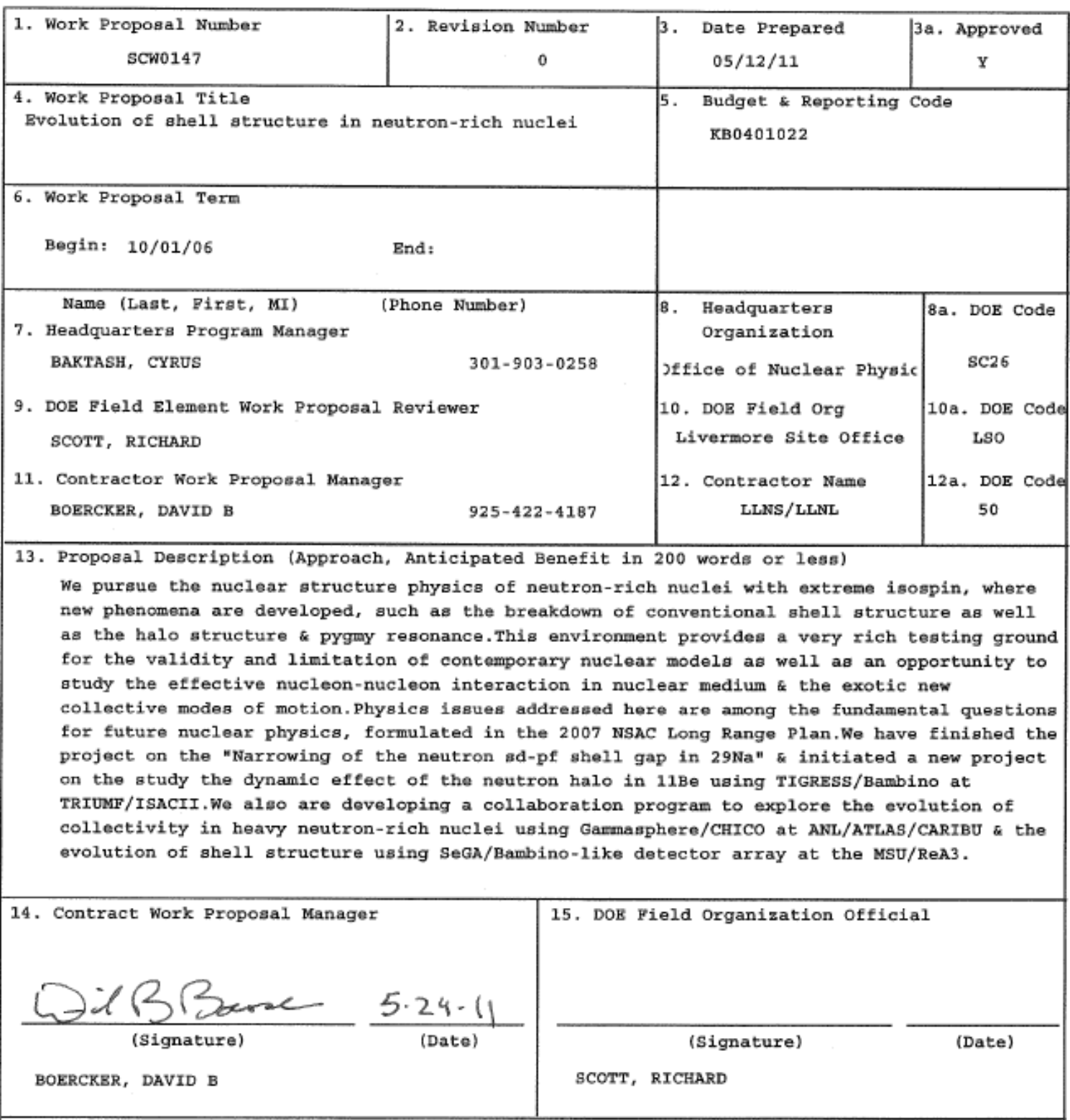

16. Detail Attachments: (See Attachments)

\begin{tabular}{|c|c|c|c|}
\hline$\overline{\mathrm{x}}_{\mathrm{b}}^{\mathrm{a}}$. & $\begin{array}{l}\text { Pacility Requirements } \\
\text { Publications }\end{array}$ & $\frac{\mathrm{x}}{\mathrm{g}} \mathrm{g}$. & $\begin{array}{l}\text { Technical progress } \\
\text { Future Accomplishments }\end{array}$ \\
\hline $\begin{array}{r}\mathrm{x} c \\
\mathrm{~d} .\end{array}$ & $\begin{array}{l}\text { Purpose } \\
\text { Background }\end{array}$ & $\underline{\mathrm{x}} \mathrm{h}$. & $\begin{array}{l}\text { Rltnshp to Other Proj } \\
\text { NEPA Requirements }\end{array}$ \\
\hline$\underline{x}$ e. & Approach & j. & Milestones \\
\hline
\end{tabular}

k. Deliverables
1. Perf. Measures/expect.
m. Ess.H Considerations
n. Human/Animal Subjects
o. Security Requirements
p. Other (Specify)

Note: Rates are subject to change due to changes in program funding levela, compliance requirements, and cost accounting practices. 
WORK PROPOSAL REQUIREMENTS FOR OPERATING/CAPITAL EQUIPMENT

OBLIGATIONS AND COSTS

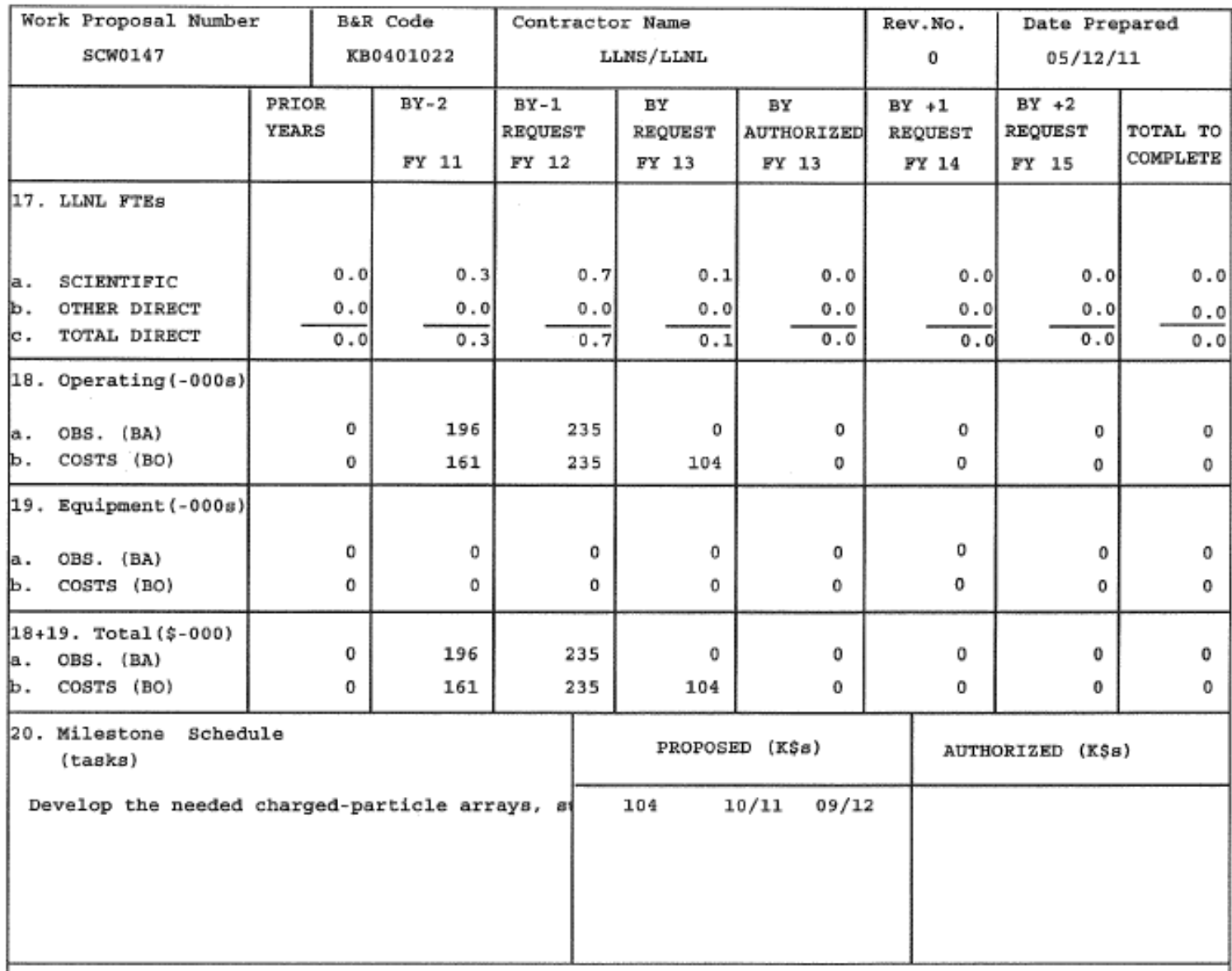

21. Reporting Requirements (Description)

I. Continue the study on the shell evolution in neutron-rich Na-Mg isotopes with N near 20. II. Develop the needed charged-particle arrays, such as the Bambino-like detector array for SeGA at MSU and CHICO2 for GRETINA.

III. Bring the research program on neutron-rich nuclei to the upcoming radioactive beam facilities at ANL (ATLAS/CARIBU) and MSU (ReA3/12).

IV. Prepare the experiment to study the dynamic neutron halo effect in $11 B e$.

Principal Investigators:

WU, CHING-YEN

$925-423-9589$ 


\section{0.c Purpose:}

The nature of the nuclear force binding stable and exotic nuclei as well as the origin of simple patterns in complex nuclei are among the fundamental issues for future nuclear physics, formulated in the 2007 NSAC Long Range Plan. Developing and designing specific experiments, in collaboration with our colleagues from universities and national laboratories around the world, to address these issues is the main goal of this experimental nuclear physics program.

Our goals for FY12 are to:

- Complete the CHICO2 project and begin the scientific program by coupling CHICO2 to GRETINA or Gammasphere.

- Begin the scientific program using SeGA/Bambino-like detector array by proposing the study of electromagnetic properties beyond the first excited state in ${ }^{30} \mathrm{Na}$ to test the Monte Carlo shell-model predictions using a Hamiltonian that includes the configuration mixing between the normal $(s d)$ and intrude (pf) shells.

- Complete the data analysis for the ${ }^{11} \mathrm{Be}$ experiment if it is fielded successfully in FY11 at TRIUMF/ISACII using TIGRESS/Bambino. The goal of this experiment is to study the dynamic effect of neutron halo in ${ }^{11} \mathrm{Be}$ by measuring the E1 strengths to both the bound excited state and the continuum.

- Field the joint experiments with our collaborators from universities and national laboratories at TRIUMF/ISAC II using TIGRESS/Bambino, at ANL/ATLAS using Gammasphere/CHICO, and at MSU/ReA3 using SeGA/Bambino-like detector array. 


\section{- 20.e Approach:}

We pursue our scientific goals by studying the evolution of shell structure and collectivity in exotic neutron-rich nuclei using the most advanced instrumentations and accelerator facilities, available both nationally and internationally, in the field of lowenergy nuclear physics. These efforts are leveraged by our actively collaborating with scientists from many institutions. We organize as well as participate in national and international workshops, and are heavily engaged in the activities of nuclear physics community. We develop the experimental programs that are at the forefront of nuclear science, and the necessary hardware as well as software to accomplish the set goals.

The $\mathrm{CHICO} 2$ project to retrofit $\mathrm{CHICO}$ with a matching position resolution for GRETINA has been funded with a strong support of the low-energy nuclear physics community. The effort includes the replacement of the cathode board with a pixelated board of $1^{\circ}$ resolution for both $\theta$ and $\phi$ coordinates. The proof-of-principle on this pixelated board was carried out at LLNL and presented in the FRIB Equipment Workshop held at MSU in Feb 2010 and Fourth TIGRESS Science Workshop held at SFU in July 2010. In addition, the effort will be made to develop a new generation of amplifier based on the successful model used for our research activity at LANL and a new front-end DAQ based on the VME platform to meet the modern standard.

A new collaboration, JANUS (Joint Array for Nuclear Structure), has been formed with scientists from LLNL, MSU, and U. of Rochester. This joint effort is to initiate a new research program to study the physics of exotic nuclei, which includes the evolution of shell structure in neutron-rich nuclei at MSU/ReA3/12 facility. The development of a Bambino-like charged-particle detector array for SeGA to meet the research demand will be carried out in LLNL. A letter-of-intent addressing the proposed JANUS research program, submitted to the MSU program advisory committee, was given a high mark and one of the proposed experiment could be the first run for the inauguration of ReA3/12.

For our research activity on the applied nuclear science, the focus is to study the neutroninduced reactions on actinides using the detector arrays such as DANCE and Chi-Nu at LANL/LANSCE together with the charged-particle detectors developed by LLNL. These activities with a tie close to Stockpile Stewardship and AFCI programs, are related to the measurements of the neutron-capture cross section as well as the fission neutron spectrum and prompt $\gamma$ rays in fission.

Key personnel:
C.Y. Wu; PI
E. Kwan; PD 


\section{0.f Technical Progress}

- The $\mathrm{CHICO} 2$ proposal to retrofit $\mathrm{CHICO}$ with a matching position resolution for GRETINA has been submitted and approved.

- The proof-of-principle work on the pixelated PPAC was successfully carried out, which is the basis for the $\mathrm{CHICO} 2$ proposal.

- Double-side segmented silicon detectors for the JANUS collaboration have been ordered and received. They will form the charged-particle detector array for the SeGA array at MSU.

- Field the joint scheduled experiments at CERN/ISOLDE to measure the octupole collectivity in ${ }^{224} \mathrm{Ra}$. This and other proposed experiments, aiming at the measurement of octupole collectivity in Rn, Fr and Ra with $\mathrm{A} \sim 220$, are necessary to interpret EDM measurements in terms of time-reversal violating interactions.

- Current status of experiments led by our collaborators:

1. Nuclear structure of ${ }^{242} \mathrm{Am}$ via Coulomb excitation of ${ }^{242 \mathrm{~m}} \mathrm{Am}$, fielded in Aug 2005 at ANL/ATLAS using Gammsphere/CHICO. The results were published in Phys. Rev. C 82, 004319 (2010). (U. of Rochester)

2. Population mechanism of $16^{+}$isomer in ${ }^{178} \mathrm{Hf}$, fielded in Aug 2008 at ANL/ATLAS using Gammasphere/CHICO. The data analysis is in progress. (U. of Rochester)

3. Nuclear structure of ${ }^{236} \mathrm{~Np}$ via the one-neutron transfer reaction between ${ }^{237} \mathrm{~Np}$ and ${ }^{116} \mathrm{Sn}$, fielded in Aug 2008 at ANL/ATLAS using Gammasphere/CHICO . The data analysis is in progress. (ANL)

4. Resonances above the $\alpha$ threshold in ${ }^{22} \mathrm{Mg}$ via the inverse $\left(\mathrm{p}, \mathrm{p}^{\prime}\right)$ reaction with ${ }^{21} \mathrm{Na}$ beam, fielded in Dec 2008 at TRIUMF/ISAC II using TIGRESS/Bambino. The data analysis is in progress. (U. of York)

5. Measurement of octupole collectivity in ${ }^{224} \mathrm{Ra}$ using Coulomb excitation, fielded Aug - Sep 2010 at CERN/REX-ISOLDE using the MINIBALL array. The data analysis is in progress. (U. of Liverpool) 


\section{20.h Relationship to Other Projects:}

We have a very close working relationship with the local theory group. For example, the successful defense of the proposal to study the dynamic effect of neutron halo in ${ }^{11} \mathrm{Be}$, can be partially attributed to the effort of two local theorists. Peter Navratil made the calculations under the framework of $a b$ initio no-core shell model for the electromagnetic properties of bound states and the continuum in "Be and Neil Summers made the cross section calculations under the framework of continuum discretized coupled channel method for the interaction between ${ }^{11} \mathrm{Be}$ and ${ }^{196} \mathrm{Pt}$ at low bombarding energy. Their effort on this proposal is tied very closely to their own projects. They also will join the effort in the data analysis and the interpretation of the final results. This approved experiment has been scheduled in June - July 2011 at TRIUMF/ISACII using TIGRESS/Bambino.

This project has a close relationship with our activities on the applied nuclear science. We adopt a similar design and fabrication principle of charged-particle detectors used in the basic nuclear science, to build the detector systems for the need of both WCI and NA22 programs. For example, a low mass fission counter with multiple PPAC's was designed and fabricated in LLNL to generate a fast and clean trigger for the Chi-Nu array at LANL to measure the fission neutron spectrum. A new compact PPAC to detect the fission fragments was designed and fabricated in LLNL, under NA22 funding, for the DANCE array at LANL to study the neutron-capture and neutron-induced fission reactions. A new generation of fast amplifier for PPAC developed under the WCI funding will be adopted for the approved $\mathrm{CHICO} 2$ charged particle detector array. This is a good case to demonstrate the cross-fertilization between the basic and applied nuclear science. 


\section{Publications in the referred journals (2005 - present):}

27. A.B. Hayes et al., "Coulomb excitation of a ${ }^{242} \mathrm{Am}$ isomeric target: E2 and E3 strengths, rotational alignment, and collective enhancement", Phys. Rev. C 82, 004319 (2010)

26. A.M. Hurst, C.Y. Wu et al., "Rotational alignments in ${ }^{235} \mathrm{~Np}$ and the possible role of $j_{15 / 2}$ neutrons", Phys. Rev. C 81, 014312 (2010)

25. R. Kanungo et al., "Structure of states in ${ }^{12} \mathrm{Be}$ via the ${ }^{11} \mathrm{Be}(\mathrm{d}, \mathrm{p})$ reaction", Phys. Lett. B $682,391(2010)$

24. M.A. Schumaker et al., "Coulomb excitation of the proton-dripline nucleus ${ }^{20} \mathrm{Na"}$, Phys. Rev. C 80, 044325 (2009)

23. S.A. Sheets et al., "Test of the statistical model in ${ }^{96} \mathrm{Mo}$ with the $\mathrm{BaF}_{2} \gamma$ calorimeter DANCE array", Phys. Rev. C 79, 024301 (2009)

22. A.M. Hurst, C.Y. Wu et al., "Narrowing of the neutron $s d-p f$ shell gap in ${ }^{29} \mathrm{Na}^{\prime \prime}$, Phys. Lett. B 674, 168 (2009)

21. U.S. Tandel et al., "Collective oblate rotation at high spins in neutron-rich ${ }^{180} \mathrm{Hf}$ ", Phys. Rev. Lett. 101, 182503 (2008)

20. M.A. Schumaker et al., "Coulomb excitation of radioactive ${ }^{21} \mathrm{Na}$ and its stable mirror ${ }^{21} \mathrm{Ne}^{2}$, Phys. Rev. C 78, 044321 (2008)

19. M. Wiedeking et al., "Intruder excitations in ${ }^{35} \mathrm{P}$ ", Phys. Rev. C 78, 037302 (2008)

18. W.D. Kulp et al., "Search for intrinsic collective excitations in ${ }^{152} \mathrm{Sm}$ ", Phys. Rev. C 77, 061301(R) (2008)

17. A.B. Hayes, et al., "Spin dependence of $K$-mixing, strong configuration mixing and electromagtice properties of ${ }^{178}$ Hf", Phys. Rev. C 75, 034308 (2007)

16. E. Ngijoi-Yogo, et al., "Collective rotation and vibration in neutron-rich ${ }^{180,182} \mathrm{Hf}$ nuclei", Phys. Rev. C 75, 034305 (2007)

15. B.R. Beck et al., "Energy splitting of the ground-state doublet in the nucleus ${ }^{229} \mathrm{Th}$ ", Phys. Rev. Lett. 98, 142501 (2007)

14. C. Wheldon et al., "Observation of an isomeric state in ${ }^{197}$ Au", Phys. Rev. C 74, 027303 (2006)

13. C.Y. Wu et al., "Evidence for possible shape transitions in neutron-rich Ru isotopes: spectroscopy of ${ }^{109,110,111,112}$ Ru", Phys. Rev. C 73, 034312 (2006)

12. A. B. Hayes, et al., "Breakdown of K selection ${ }^{178} \mathrm{Hf}$ ", Phys. Rev. Lett. 96, 042505 (2006)

11. J. Srebrny et al., "Experimental and theoretical investigations of quadrupole collective degrees of freedom in "Ru", Nucl. Phys. A 766, 25 (2006)

10. A. Schiller et al., "Low-energy M1 excitation mode in ${ }^{172} \mathrm{Yb}$ ", Phys. Lett. B 633, 225 (2006)

9. D. Cline, A.B. Hayes, and C.Y. Wu, "Nuclear structure implications for controlled energy release of isomers", J. of Modern Optics, 52, 2411 (2005)

8. T.M. Shneidman et al., "E2 transitions between positive- and negative-parity states of the ground-state alternating-parity bands", Eur. Phys. J. A 25, 387 (2005)

7. R.S. Chakrawarthy et al., "Discovery of a new $2.3 \mathrm{~s}$ isomer in neutron-rich ${ }^{174} \mathrm{Tm}$ ", Eur. Phys. J. A 25,125 (2005)

6. N. Fotiades et al., "High-spin states in $\mathrm{N}=50{ }^{85} \mathrm{Br}$ and ${ }^{87} \mathrm{Rb}$ nuclei", Phys. Rev. C 71,064312 (2005)

5. M.S. Johnson et al., "Quasicontinuous decay and properties of superdeformed excitations in ${ }^{195} \mathrm{~Pb} "$, Phys. Rev. C 71, 044310 (2005)

4. S. Zhu et al., "Strength of octupole correlations in the actinides: contrasting behavior in the isotones ${ }^{237} \mathrm{U}$ and ${ }^{230} \mathrm{Pu}$ ", Phys. Lett. B 618, 51 (2005) 
3. I. Ahmad, et al., "Search for x-ray induced decay of the $31-\mathrm{yr}$ isomer of ${ }^{178} \mathrm{Hf}$ using synchrotron radiation", Phys. Rev. C 71, 024311 (2005)

2. M.S. Johnson et al., "Population of superdeformed excitations in ${ }^{198}$ Po", Phys. Rev. C 71 , $024317(2005)$

1. W.D. Kulp et al., "Identification of a pairing isomeric band in ${ }^{152} \mathrm{Sm}$ ", Phys. Rev. C 71, 041303(R) (2005)

\section{Conference Proceedings:}

18. C.Y. Wu et al., "Pixelated PPAC for the $\gamma$-ray energy tracking arrays", http://meetings.aps.org/link/BAPS.2010.DNP.JF.6

17. A.M. Hurst et al., "Narrowing of the neutron sd-pf shell gap in ${ }^{29} \mathrm{Na}$ : First in-beam $\gamma$-ray spectroscopy results using TIGRESS at ISAC II", KERNZ08

16. M.A. Schumaker et al., "Coulomb excitation of radioactive ${ }^{20,21} \mathrm{Na}$ ", Eur Phys. J. A. (2009), DOI 10.1140/epja/i2009-10816-4

15. M.A. Schumaker et al., "First results with TIGRESS and accelerated radioactive ion beams from ISAC: Coulomb excitation of ${ }^{20,21,29} \mathrm{Na"}$, CPI099, Application of Accelerators in Research and Industry: $20^{\text {th }}$ Int. Conf., edited by F.D. McDaniel and B.L. Doyle (2009), p. 754

14. M.A. Schumaker et al., "Coulomb excitation of radioactive ${ }^{21} \mathrm{Na",}$ Proceedings of the International Nuclear Physics Conference (INPC 2007), (Tokyo 2007) V2, p.362

13. P. Chowdhury et al., "Competing shapes and alignments in neutron-rich Hf nuclei", Proceedings of the 2nd International Conference on Frontiers in Nuclear Structure, Astrophysics, and Reactions, edited by P. Demetriou, R. Julin, and S.V. Harissopulos (American Institute of Physics, New York, 2008), p. 265

12. P.E. Garrett et al., "The TRIUMF nuclear structure program and TIGRESS", Nucl Instr. and Methods in Phys. Res. B 261, 1084 (2007)

11. A.B. Hayes et al., "Coulomb excitation of the ${ }^{242 \mathrm{~m}} \mathrm{Am}$ isomer", Laser Physics 17, 745 (2007)

10. M.A. Stoyer et al., "Spectroscopy of neutron-rich $\mathrm{Pd}$ and $\mathrm{Cd}$ isotopes near $\mathrm{A}=120$ ", Nucl. Phys. A787, 455c (2007)

9. G.C. Ball et al., "TRIUMF-ISAC Gamma-Ray Escape-Suppressed Spectrometer (TIGRESS): a versatile tool for radioactive beam physics", Nucl. Phys. A787, $118 \mathrm{c}$ (2007)

8. P.E. Garrett et al., "The TRIUMF nuclear structure program and TIGRESS", Nucl. Instrum. Methods in Phys. Res. B 261, 1084 (2007)

7. G.C. Ball et al., "TRIUMF-ISAC Gamma-Ray Escape-Suppressed Spectrometer (TIGRESS): a versatile tool for radioactive beam physics", Nucl. Phys. A 787, 118c (2007)

6. J.A. Becker and R.O. Nelson, "Neutron Induced Reactions and Spectroscopy with GEANIE", ND2004, International Conference on Nuclear Data for Science and Technology (Santa Fe, NM, September 2004), AIP Conf. Proc. 769, pp 748-753 (2005)

5. R. O. Nelson et al., "Cross-Section Standards for Neutron-Induced Gamma-Ray Production in the MeV Energy Range", ND2004, International Conference on Nuclear Data for Science and Technology (Santa Fe, NM, September 2004), AIP Conf. Proc. 769, pp 838-841 (2005)

4. N. Fotiades et al., "Cross sections for gamma-ray production in the ${ }^{191} \operatorname{Ir}(\mathrm{n}, \mathrm{xn}, \gamma)$ reactions", ND2004, International Conference on Nuclear Data for Science and Technology (Santa Fe, NM, September 2004), AIP Conf. Proc. 769, pp 898-901 (2005)

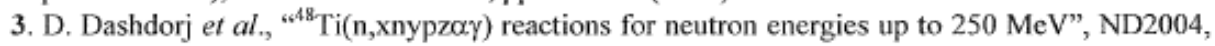
International Conference on Nuclear Data for Science and Technology (Santa Fe, NM, September 2004), AIP Conf. Proc. 769, pp 1035-1038 (2005)

2. W.B. Walters et al., "Structure and decay of neutron-rich nuclides in the $115 \leq \mathrm{A} \leq 138$ mass region and $r$-process nucleosynthesis", International Conference on Nuclei at Limits (Argonne, 
IL, July 2004), AIP Conf. Proc. 764, p. 335 (2005)

1. D. Ward et al., "Aspects of the Coriolis interaction in ${ }^{235} \mathrm{U}$ ", International Conference on Nuclei at Limits (Argonne, IL, July 2004), AIP Conf. Proc. 764, p. 263 (2005)

\section{Invited Talks:}

21. C.Y. Wu, "Pixelated PPAC for the $\gamma$-ray energy tracking arrays", Fourth TIGRESS Science Workshop, SFU, Burnaby, BC, July $10-12,2010$

20. C.Y. Wu, "Report on CHICO II development for exotic beam physics", FRIB

Equipment Workshop, MSU, East Lansing, MI, Feb 20 - 22, 2010

19. C.Y. Wu, "Radioactive nuclear beam experiments at TRIUMF", ACS $238^{\text {th }}$ National

Meeting, Washington, DC, Aug 16 - 20, 2009

18. C.Y. Wu, "Evolution of nuclear structure for nuclei with extreme isospin", RIA/FRIB Workshop at Argonne National Laboratory, May 30 - 31, 2009

17. C.Y. Wu, "Low mass fission chamber", LANL-LLNL Fission Workshop, Los Alamos National Laboratory, Los Alamos, NM, Feb 3 - 6, 2009

16. A.M. Hurst, "Narrowing of the neutron $s d-p f$ shell gap in ${ }^{29} \mathrm{Na}^{\prime}$, International Conference on Interfacing Structure and Reactions at the Centre of the Atom, Queenstown, New Zealand, Dec 1 - 5, 2008

15. A.M. Hurst, "Narrowing of the neutron $s d-p f$ shell gap in ${ }^{29} \mathrm{Na}^{\prime}$, ISOLDE Workshop and Users Meeting, CERN, Geneva, Swiss, Nov 17 - 19, 2008

14. C.Y. Wu, "Overview of LLNL experimental program", Cross Section Evaluation Working Group Meeting, Brookhaven National Laboratory, Upton, NY, Nov 4 - 6, 2008 13. C.Y. Wu, "SuperCHICO; a pixelated gas avalanche counter for the $\gamma$-ray energy tracking arrays", Third TIGRESS Science Workshop: 2010 and beyond, Lawrence Livermore National Laboratory, Livermore, CA, Feb 21 - 22, 2008

12. A.M. Hurst, "Low-energy Coulomb excitation of radioactive ${ }^{29} \mathrm{Na}^{\circ}$, Third TIGRESS Science Workshop: 2010 and beyond, Lawrence Livermore National Laboratory, Livermore, CA, Feb $21-22,2008$

11. C.Y. Wu, "Experimental program at LLNL", Cross Section Evaluation Working Group Meeting, Brookhaven National Laboratory, Upton, NY, Nov 6 - 8, 2007

10. C.Y. Wu, "Spectroscopy and $(\mathrm{n}, \mathrm{f})$ cross section for ${ }^{242 \mathrm{~m}} \mathrm{Am}$ ", Nuclear Reactions on Americium Workshop, Santa Fe, Sep 18 - 19, 2007

9. C.Y. Wu, "Nuclear structure with Gammasphere/CHICO and beyond", Excellence in Basic and Applied Nuclear Science, Monterey, CA, June 25 - 26, 2007

8. C.Y. Wu, "Nuclear structure with Gammasphere/CHICO and beyond", APS April Meeting 2007, Jacksonville, FL, April 14 - 17, 2007

7. D. Cline and C.Y. Wu, "Nuclear Structure studies of both stable and exotic nuclei", International Conference on Nuclear Structure 2006, Oak Ridge National Lab, July 24-28, 2006

6. C.Y. Wu, J.A. Becker, and D. Cline, "Bambino/SuperCHICO/TIGRESS at TRIUMF/ISAC", TIGRESS Scientific Roadmap Meeting, Saint Mary's University, Halifax, NS, June 16 - 17, 2006

5. C.Y. Wu, J.A. Becker, and D. Cline, "CHICO/Bambino/Gammasphere at ANL", ATLAS User Workshop, Argonne National Lab, Chicago, April 7, 2006

4. C.Y. Wu, J.A. Becker, and D. Cline, "Bambino at TIGRESS", Workshop for a $4 \pi$ Silicon Detector Array for TIGRESS, Golden, CO, Feb 24-25, 2006 
3. C.Y. Wu, J.A. Becker, and D. Cline, "SuperCHICO at GRETINA and TIGRESS", Workshop on Auxiliary Detectors for GRETINA, Jan 28-29, 2006

2. J.A. Becker, "The GEANIE $\mathrm{n}+{ }^{89}$ Y Cross Section Set at LLNL", ILWOG 39, Los Alamos, NM, May 2005

1. J.A. Becker, "Experiments on Atomic-Nuclear Coupling", 12th Conference on Capture Gamma-ray and Related Topics, Notre Dame, Indiana, September 2005 
U.S. DEPARTMENT OF EMERGY PIELD WORK PROPOSAL - FY 2013

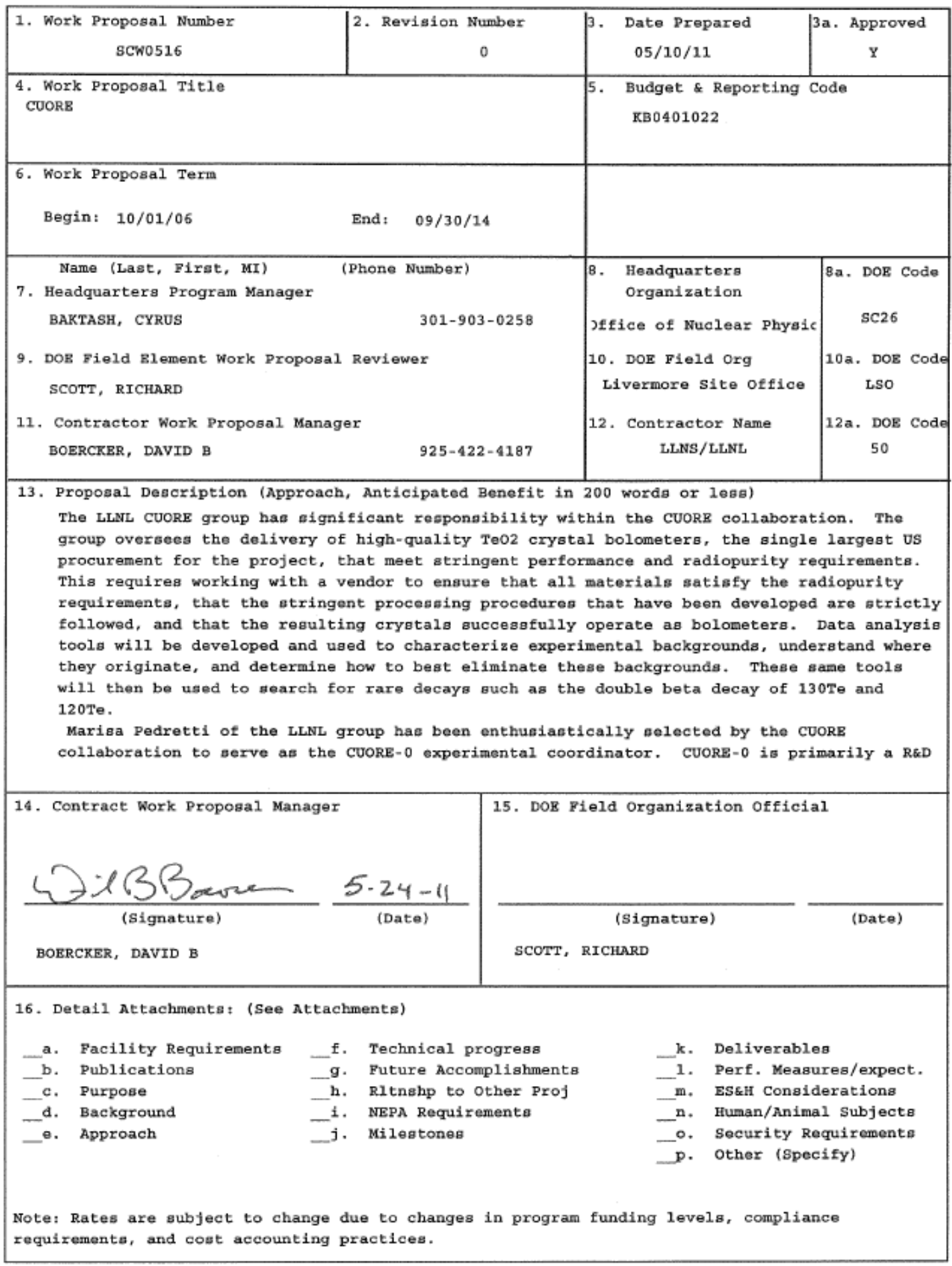


WORK PROPOSAL REQUIREMENTS FOR OPERATING/CAPITAL EQUIPMENT OBLIGATIONS AND COSTS

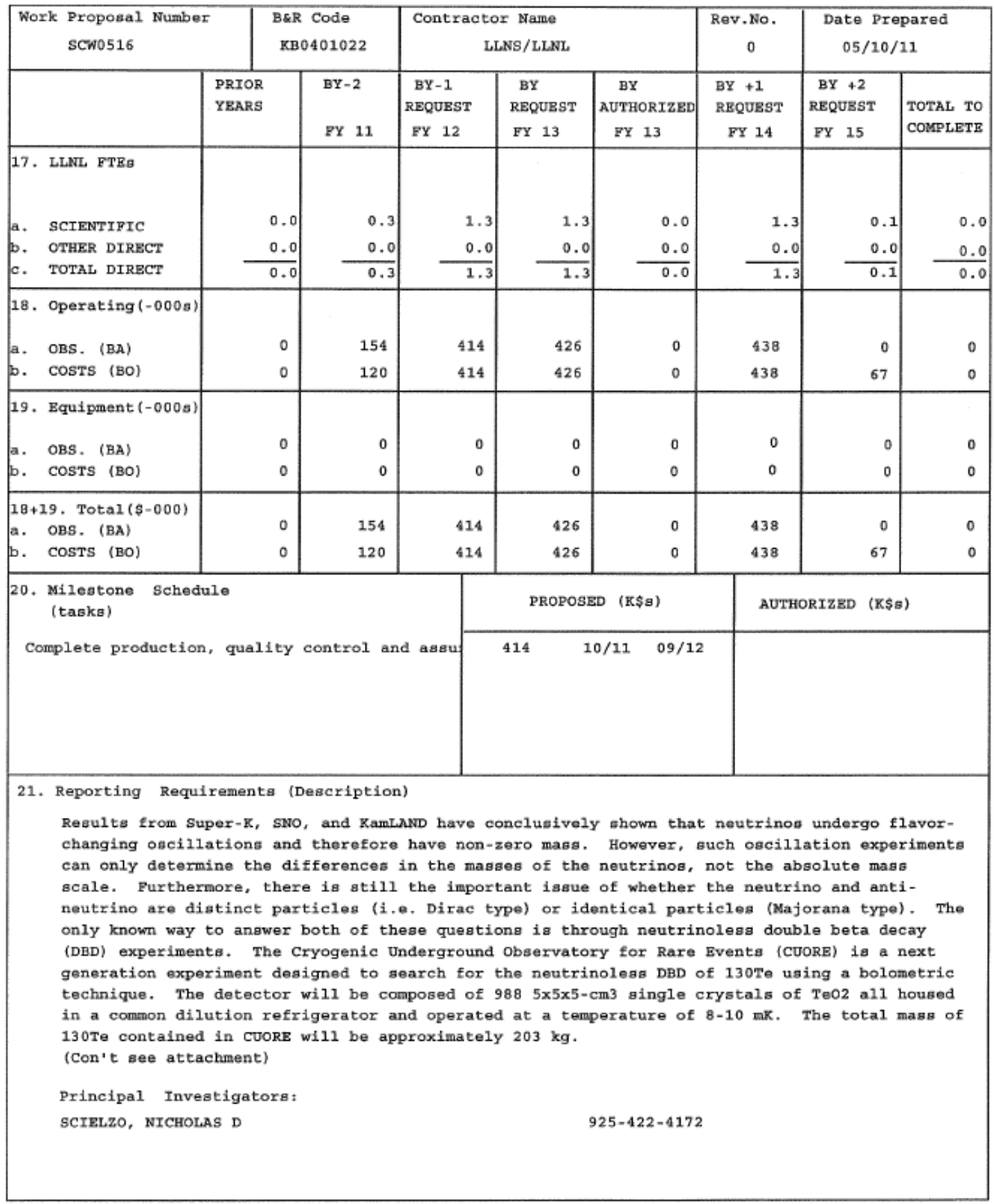


U.s. DEPARTMENT OF BNBRGY FIELD WORK PROPOSAL - FY 2013

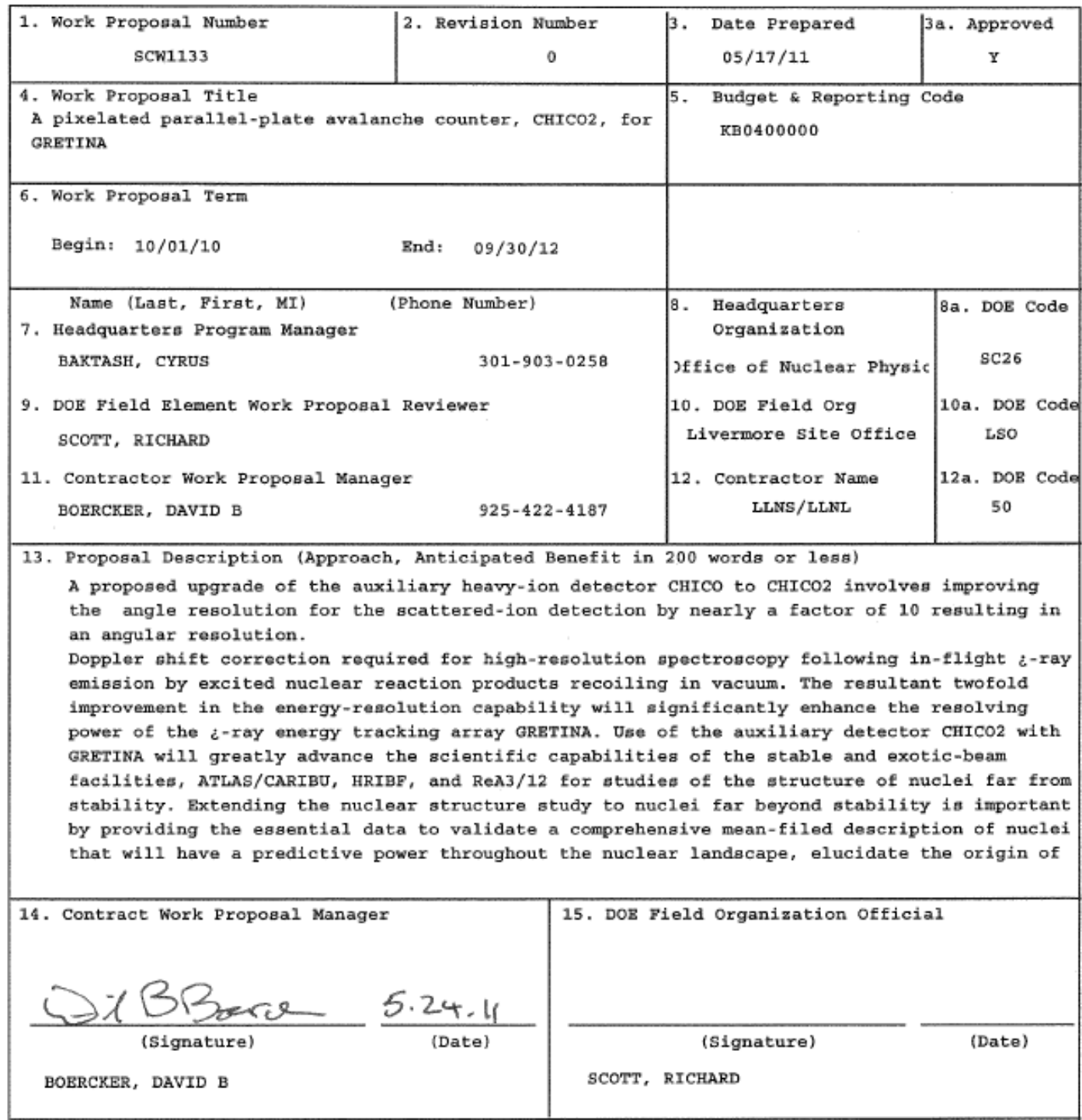

16. Detail Attachments: (See Attachments)

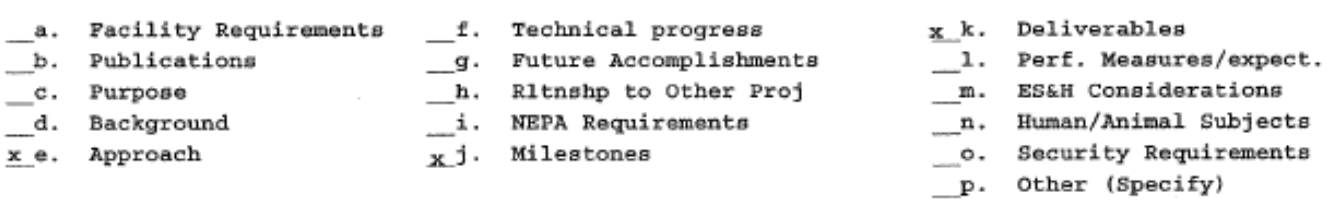

Note: Rates are subject to change due to changes in program funding levels, compliance requirements, and cost accounting practices. 
WORK PROPOSAL REQUIREMENTS FOR OPERATING/CAPITAL EQUIPMENT

OBLIGATIONS AND COSTS

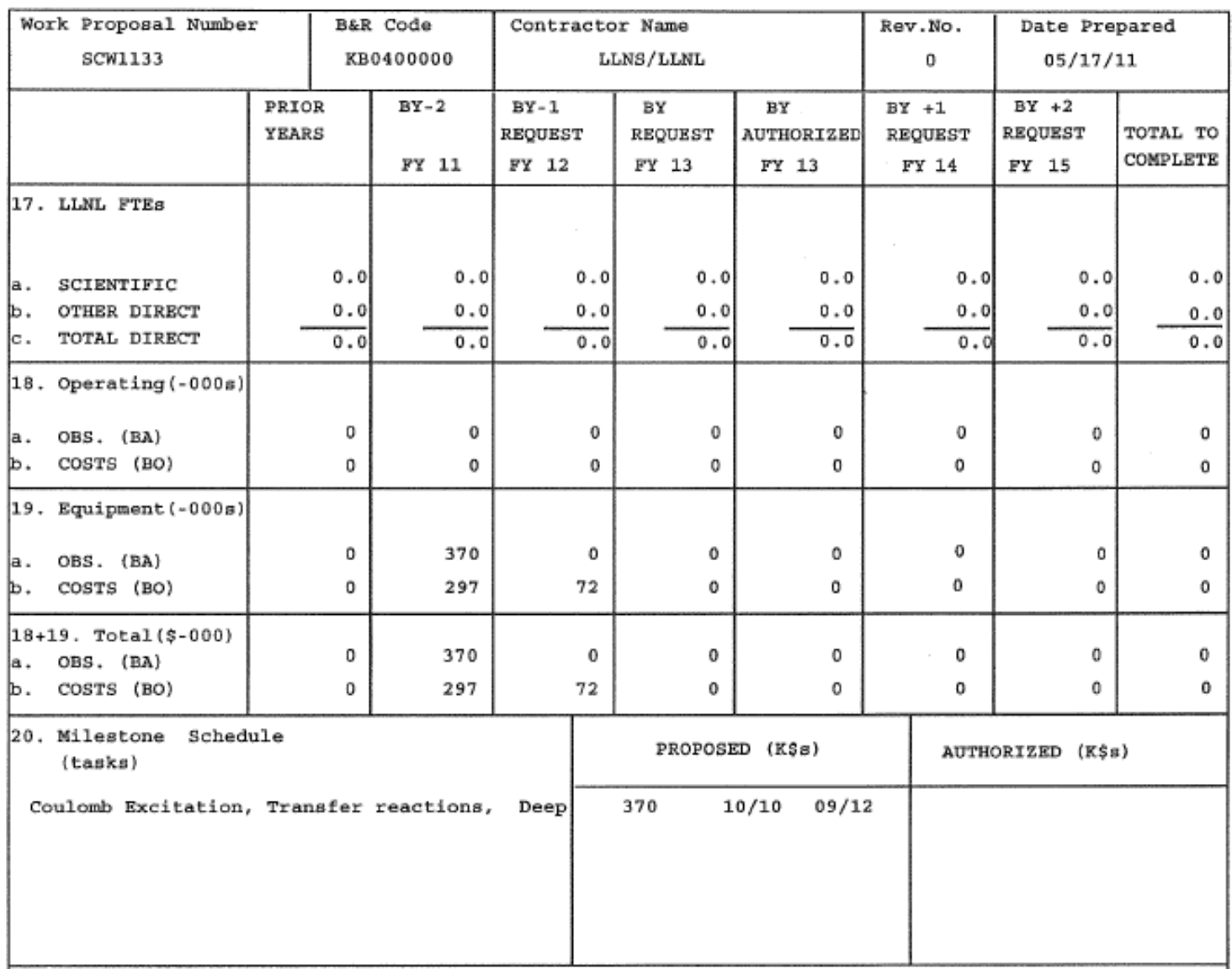

21. Reporting Requirements (Description)

See attached

Principal Investigators:

WU, CHING-YEN

$925-423-9589$ 


\title{
SCW1133 attachment
}

\begin{abstract}
:
A proposed upgrade of the auxiliary heavy-ion detector $\mathrm{CHICO}$ to $\mathrm{CHICO} 2$ involves improving the $\phi$ angle resolution for the scattered-ion detection by nearly a factor of 10 resulting in an angular resolution of $\Delta \theta=\Delta \phi=1^{\circ}$. This angle resolution allows for precise Doppler shift correction required for high-resolution spectroscopy following in-flight $\gamma$ ray emission by excited nuclear reaction products recoiling in vacuum. The resultant twofold improvement in the energy-resolution capability will significantly enhance the resolving power of the $\gamma$-ray energy tracking array GRETINA. Use of the auxiliary detector CHICO2 with GRETINA will greatly advance the scientific capabilities of the stable and exotic-beam facilities, ATLAS/CARIBU, HRIBF, and ReA3/12 for studies of the structure of nuclei far from stability. Extending the nuclear structure study to nuclei far beyond stability is important by providing the essential data to validate a comprehensive mean-filed description of nuclei that will have a predictive power throughout the nuclear landscape, elucidate the origin of elements in the cosmos, provide an understanding of matter in the crust of neutron stars, and formulate a scientific foundation for innovative applications of nuclear science to society.
\end{abstract}

The $\mathrm{CHICO} / \mathrm{Gammasphere} \mathrm{research} \mathrm{program} \mathrm{has} \mathrm{demonstrated} \mathrm{that} \mathrm{CHICO} 2$ is ideally suited for nuclear structure studies by exploiting the heavy-ion binary reactions; such as Coulomb excitation, transfer reactions, deep-inelastic reactions, and fission. The principal investigators have long been leaders in exploiting these experimental techniques for nuclear structure studies with stable and radioactive beams. The above heavy-ion binary nuclear reactions typically have the largest cross sections, which, when coupled with high detection efficiencies for both the reaction-products and deexcitation $\gamma$-rays, makes viable the exploration of quadrupole, octupole, and pairing collective modes of motion, as well as the ability to probe magnetic moments, single-particle structure and unusual isomeric configurations, all to the farthest limits of isospin and mass. The CARIBU/ATLAS, $\mathrm{HRIBF}$, and ReA3 facilities have complementary capabilities in providing stable beams throughout the complete periodic table at energies above the Coulomb barrier as well as exotic beams ranging from the $\mathrm{N} \sim \mathrm{Z}$ line, for light to medium mass nuclei, to the whole mass range of neutron-rich fission fragments. On-site access to transactinide target production and handling capabilities will extend studies towards the transactinide limit of stability. CHICO2, used as an auxiliary detector with either GRETINA or Gammasphere, will provide the opportunity to exploit these facilities to make the significant advances in understanding of nuclear structure outlined in the 2007 Long Range Plan for Nuclear Science.

CHICO was designed to match the Gammasphere angular resolution which is limited by the size of the individual Ge detectors. As a consequence CHICO has insufficient azimuthal angle resolution to achieve the precise Doppler correction required by $\gamma$-ray tracking arrays such as GRETINA. Prototype x-y checkerboard geometry of pixels for the cathode using the delay-line technique to achieve the angular resolution required by GRETINA, was developed at LLNL in FY10. The subsequent test together with a new 
generation of fast amplifiers was highly successful and provided the proof of principle required for this proposal. The linearity of the cathode outputs is excellent and the position resolution better than $2 \mathrm{~mm}$ is achieved.

For $\mathrm{CHICO} 2$, pixelated cathodes with $\theta-\phi$ sensing for the same angular coverage as CHICO and fast amplifiers will be developed in LLNL, then assembled and tested in U. of Rochester. A new VME based data acquisition system will be developed to match that of GRETINA. The schedule involves 12 months for production of the pixelated boards, transmission lines, amplifiers, plus production of the GRETINA hemisphere at Rochester. This will be followed by six months for assembly and testing at Rochester followed by a month to interface with digital Gammasphere plus a commissioning run at ATLAS. Assuming a starting date of October 2010 then commissioning will occur by May 2012 assuming availability of access to a test beam. A technical publication on this development is anticipated.

The proposal requests the fund for hardware procurement and the cost of manpower will be provided by our existing funds in U. of Rochester (NSF), LLNL (DOE), and LBNL (DOE).

\section{PROJECT OBJECTIVE:}

As mentioned earlier, the current development of new capability is to extend the nuclear structure study to nuclei far beyond the stability, which is essential to develop a comprehensive nuclear model with predictive power. Examples of the scientific goals of such studies with CHICO2/GRETINA are given below.

Coulomb Excitation: Coulomb excitation is the preeminent probe of collective modes in nuclear structure and the cross sections are large; consequently it is an ideal probe for exploiting low-intensity exotic beams. (1) Coulomb excitation of neutron-rich fission fragments in the $\mathrm{A} \sim 100$ and $\sim 130$ mass regions will probe the rapid changes in deformation and shape coexistence that occur in these nuclei. Coulomb excitation will determine the $\mathrm{E} \lambda$ strengths, providing a sensitive probe of collective motion, while the deorientation effect will measure excited-state magnetic moments as a probe of the shell structure. Such studies will elucidate the evolution of shell and collective modes with increasing neutron excess. (2) A longstanding question in nuclear physics is the role of octupole correlations and reflection asymmetry in nuclear structure. Octupole correlations are especially strong in nuclei where an intruder state comes close to the Fermi level giving rise to $(l, j: l-3, j-3)$ particle-hole configurations at low excitation energies. These configurations are found near $Z=34,56$, and 88 and $N=34,56,88$ and 134 leading to reflection asymmetry phenomena such as odd-even staggering of positive and negative parity bands, parity doublets and enhanced E1 and E3 strengths. The octupole correlations will be studied in the ${ }^{144} \mathrm{Ba}$ region using fission-fragment beams and in the ${ }^{226} \mathrm{R}$ a region using target excitation by stable beams. (3) The successful Coulomb excitation study of ${ }^{242} \mathrm{Am}\left(\mathrm{T}_{1 / 2}=141 \mathrm{y}\right)$ illustrates that it is feasible to study Coulomb excitation of transactinide nuclei at target activity levels of several millicuries, that is, halflives longer than $50 \mathrm{y}$. There are 24 transactinide nuclei with $Z \geq 92$ that could be 
studied to high spin using CHICO2 plus Gammasphere or GRETINA providing an opportunity to probe the role of angular momentum and nuclear structure at the transactinide limits of stability.

Transfer reactions: Heavy-ion induced transfer reactions offer unique opportunities to investigate single-particle structure in nuclei. For example, heavy-ion induced neutrontransfer studies of form factors and spectroscopic strengths for collective bands in neutron-rich fission products can probe neutron enhancement in the surface region of neutron-rich nuclei. For the most intense neutron-rich beams it may be possible to probe two-neutron correlations in the surface region of nuclei by pair transfer.

Deep inelastic reactions: Deep-inelastic reactions with neutron-rich beams and targets can populate high spin states in neutron rich products. For example, using a ${ }^{238} \mathrm{U}$ target and ${ }^{64} \mathrm{Ni}$ beam, states in ${ }^{71} \mathrm{Cu}$, which has one proton and six neutrons more than the beam nucleus, have been studied recently. States with spin up to 20 have been observed in deformed nuclei. Using neutron-rich radioactive beams, the study of nuclear shell evolution and new collective modes can be extended to even more neutron-rich nuclei beyond those that can be studied with stable or radioactive beams using Coulomb excitation and knockout reactions.

However, similar to transfer reactions, the two-body nature of deep inelastic reactions requires detection of the reaction products with good angular resolution in order to perform Doppler correction to restore $\gamma$-ray energy resolution. Currently, there is no large area particle detector system which can provide the needed one degree position resolution. Thus, many current studies are carried out using a thick target to stop the products and are limited to the study of states with long life times. This method works well for isomeric states and non collective states. $\mathrm{CHICO} 2$ will broaden the studies to the collective states and the transition from closed shell nuclei to collective behavior.

Nuclear fission: $\mathrm{CHICO} 2$ is a sensitive probe of fission decay channels. Exotic-beam induced reactions could be exploited to study nuclear fission at the boundaries of nuclear stability. CHICO2 coupled to GRETINA is a very sensitive instrument to measure the $\gamma$ ray production of neutron-induced fission of actinides, which is important to the reactor fuel design relevant to the Advanced Fuel Cycle Initiative.

CHICO2 is designed as an auxiliary detector for GRETINA and is a shared instrumentation for the nuclear physics community. Therefore, it opens to anyone in the community who is interested in using this detector system for the research. 


\section{Personnel Supported by DOE/SC/NP}

\begin{tabular}{|l|c|c|}
\hline \multicolumn{1}{|c|}{ Scientist } & Job Code & $\begin{array}{c}\text { Fraction } \\
\text { support }\end{array}$ \\
\hline Nick Scielzo & FX Staff & 0.05 \\
\hline Marisa Pedretti & PD (2009-2012) & 0.75 \\
\hline Ching-Yen Wu & Staff & 0.10 \\
\hline Aaron Hurst & PD (2008-10) & 0.75 \\
\hline Elaine Kwan & PD (2010-2013) & 0.50 \\
\hline Mark Stoyer & Staff & 0.05 \\
\hline
\end{tabular}

\section{ENP Group Personnel}

\begin{tabular}{|l|c|l|}
\hline \multicolumn{1}{|c|}{ Scientist } & Job Code & \multicolumn{1}{c|}{ Major project(s) } \\
\hline Lee Bernstein & Staff & NIF nuclear science \\
\hline Darren Bleuel & FX Staff & NIF nuclear science \\
\hline Jason Burke & Staff & Surrogates/Excited states \\
\hline Mike Moran & Staff & NIF nuclear science \\
\hline Jennifer (Jo) Ressler & Staff & Non-proliferation/RIB \\
\hline Nick Scielzo & FX Staff & CUORE/Surrogates/Trapping \\
\hline Steven Sheets & FX Staff & Non-proliferation \\
\hline Wolfgang Stoeffl & Staff & NIF nuclear science \\
\hline Mark Stoyer & Group Leader & NIF nucl. Science/SHE \\
\hline Ching-Yen Wu & Staff & Exotic nuclei/RIB/Fission \\
\hline Robert Casperson & PD & Excited states/Isomers \\
\hline Andrii Chyzh & PD & Fission \\
\hline Elaine Kwan & PD & RIB/Fission \\
\hline Sarah Nelson & PD & Non-proliferation/SHE \\
\hline Marisa Pedretti & PD & CUORE \\
\hline Daniel Sayre & PD & NIF nuclear science \\
\hline Larry Ahle & Guest & Detector development \\
\hline John Becker & Retiree & Fission \\
\hline Eric Norman & Guest & Surrogates/Nuclear structure \\
\hline Ryan Yee & Grad. Student & Trapping \\
\hline
\end{tabular}




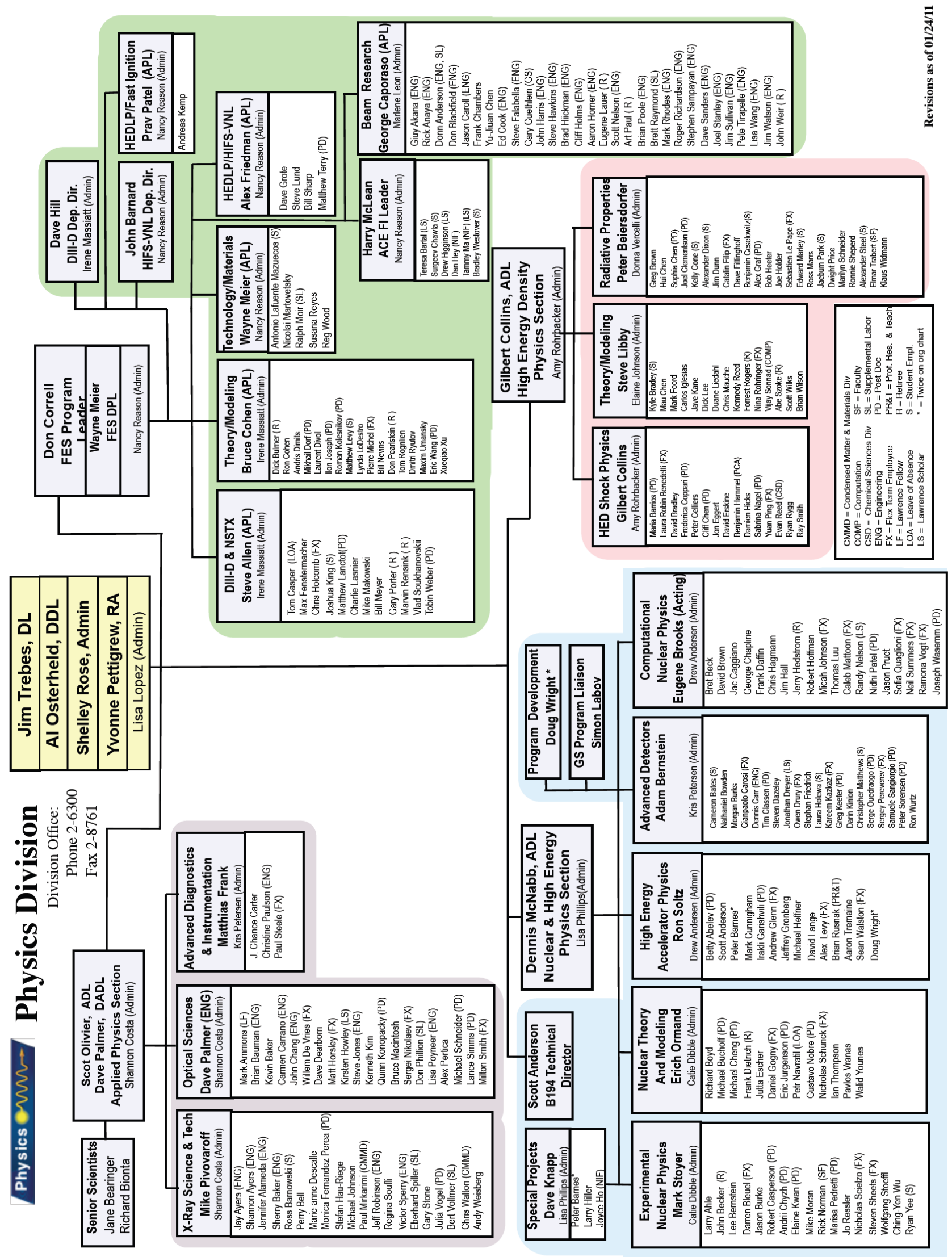


LLNL-TR-497871

\begin{tabular}{|c|c|c|c|c|c|c|c|c|c|c|c|}
\hline Laboratory Name & For each st & ff member, plea & indicate & fractio & I FTE supp & ed by $\mathrm{e}$ & fundin & rce & & & \\
\hline FY 101X & $\begin{array}{l}\text { Research: Structure, } \\
\text { reaction, \& } \\
\text { astrophysics } \\
\end{array}$ & $\begin{array}{c}\text { Experimental } \\
\text { Support: Structure, } \\
\text { reaction, \& } \\
\text { astrophysics } \\
\end{array}$ & Neutrino & Neutron & $\begin{array}{c}\text { Fund. } \\
\text { Interactions }\end{array}$ & Project & LDRD & WFO & Org Burden & Other & $\begin{array}{c}\text { Total FTEI } \\
\text { staff }\end{array}$ \\
\hline & & & & & & & & & & & 0 \\
\hline & & & & & & & & & & & 0 \\
\hline & & & & & & & & & & & 0 \\
\hline & & & & & & & & & & & 0 \\
\hline & & & & & & & & & & & 0 \\
\hline & & & & & & & & & & & 0 \\
\hline & & & & & & & & & & & 0 \\
\hline & & & & & & & & & & & 0 \\
\hline & & & & & & & & & & & 0 \\
\hline & & & & & & & & & & & 0 \\
\hline & & & & & & & & & & & 0 \\
\hline & & & & & & & & & & & 0 \\
\hline & & & & & & & & & & & 0 \\
\hline & & & & & & & & & & & 0 \\
\hline & & & & & & & & & & & 0 \\
\hline & & & & & & & & & & & 0 \\
\hline & & & & & & & & & & & 0 \\
\hline & & & & & & & & & & & 0 \\
\hline $\begin{array}{c}\text { Total FTE } \\
\text { (funding source) }\end{array}$ & 0 & 0 & 0 & 0 & 0 & 0 & 0 & 0 & 0 & 0 & 0 \\
\hline
\end{tabular}




\section{Scientific accomplishments}

Funding for the past review period is summarized in Table 1 and indicates the magnitude of the DOE/SC/NP LENP effort at LLNL.

Table 1: Funding profile for FY08-FY11.

\begin{tabular}{|c|c|c|c|c|c|}
\hline Project & PI & FY08 (K\$) & FY09 (K\$) & FY10 (K\$) & FY11 (K\$) \\
\hline Exotic Nuclei & $\mathrm{Wu}$ & 253 & 188 & $175+370^{*}$ & 175 \\
\hline CUORE & Scielzo & 73 & 158 & 175 & $175+250 * *$ \\
\hline
\end{tabular}

Based on the last four-year review in August 2007, we have focused our efforts on two areas of research, 1) the investigation of collective effects in exotic nuclei in order to understand fundamental nuclear forces and the evolution of shell structure in the nuclear landscape, and 2) the search for neutrinoless double-beta decay in order to understand fundamental properties of the neutrino. These research areas address NSAC priorities specifically under milestones 1) NS9 for 2018, to measure changes in shell structure and collective modes, from the most proton-rich to the most neutron-rich nuclei accessible, in order to improve our understanding of the nucleus, and to guide theory in every region of the theoretical roadmap; and 2) FI14 for 2017, to extend the sensitivity of searches for neutrinoless double-beta decay in selected nuclei by a factor of ten in lifetime, respectively. Thus, funding was re-apportioned to enable support of two PDs (one for each project including 0.25 FTE support by LLNL G\&A for career development activities) and to enable greater progress in both efforts through the mentoring of PDs. These efforts are supported by FWP \#'s SCW0147 and SCW0516, respectively. Significant progress was made in both of these research areas as discussed in detail below.

\section{Exploration of exotic nuclei and the collective properties of nuclei}

The nature of the nuclear force binding stable and exotic nuclei as well as the origin of simple patterns in complex nuclei are among the fundamental issues to be addressed for the future in nuclear physics, as formulated in the 2007 NSAC Long Range Plan. This provides guidance for our accelerator-based nuclear physics program.

Based on our prior work on the electromagnetic properties of collective nuclei using low-energy Coulomb excitation [1], we decided in FY08 to develop a program to extend these studies to nuclei with extreme neutron/proton ratios becoming available at modern radioactive beam facilities with a particular focus on the evolution of shell structure far from stability and the enhanced E1 strength in halo nuclei. Our activities in FY08-FY11 centered around two different thrusts. First, we developed particle-gamma coincidence techniques at both TRIUMF and MSU and performed measurements to study the collectivity of exotic nuclei by measuring low-lying transition matrix elements in the region of $\mathrm{N} \sim 8,20$ at TRIUMF. Second, we studied collective properties of heavier nuclei in quasi-inelastic reactions with our established collaboration at ANL using the Gammasphere array coupled to $\mathrm{CHICO}$, and developed $\mathrm{CHICO}$, a new instrument to 
be coupled to GRETA/GRETINA to provide kinematic reconstruction at a resolution that fully exploits the resolving power of these next-generation gamma-ray spectrometers.

\section{Probing collectivity in light nuclei with particle-gamma coincidences}

In the last proposal cycle, we developed a new capability for particle-gamma coincidence measurements at TRIUMF by coupling the TIGRESS array to a new particle tracing array called Bambino. TIGRESS is Canadian $4 \pi$ Compton-suppressed $\gamma$-ray energy tracking array and Bambino consists of two double-side segmented silicon detectors placed at both forward and backward hemispheres relative to the target position. This particle-gamma coincidence capability had been successfully deployed in FY07. In FY08-FY11 we used this new capability to measure the collectivity in selected nuclei further from stability than had been previously achieved. The $\mathrm{N} \sim 8,20$ nuclei were particularly appealing area of study because the breakdown of traditional "magic" numbers is known to occur as one moves further from stability, yet the number of nucleons is small enough so that a rich set of theoretical techniques to predict the evolution of shell structure is available. Our experience at TRIUMF with TIGRESS/Bambino has naturally led us to play a leadership role in the development of instrumentation needed by the community in the build up to FRIB. On this front, we have become active members of the JANUS collaboration (spokesperson: A. Gade of MSU). LLNL's initial role in this collaboration has been to lead the development of particle tracking capabilities for Coulomb excitation experiments at the ReA accelerator.

\section{The evolution of shell structure in Be and $\mathrm{Na}$ isotopes at TRIUMF}

The TIGRESS/Bambino setup is optimized to provide angular coverage for the detection of charged particles in low-energy radioactive beam experiments in order to measure transition strengths of low-lying excited states. TRIUMF also had excellent success in developing Be and $\mathrm{Na}$ beams, so our collaboration began investigations into the collectivity near $\mathrm{N} \sim 8,20$ with $\mathrm{Be}$ and $\mathrm{Na}$ beams. One long-term scientific goal of the LLNL group is to advance our understanding of the tail of wavefunctions in halo nuclei, e.g. the one-neutron halo nucleus ${ }^{11} \mathrm{Be}$. Precision measurements of the E1 strength in this nucleus provide insight into the development of models beyond the shell model. These plans are discussed in our FY12-15 research plan. The initial experiments of the collaboration in this region were an inverse $(\mathrm{d}, \mathrm{p})$ reaction with ${ }^{11} \mathrm{Be}$ beam in search of the halo structure in ${ }^{12} \mathrm{Be}$ [2], where the halo-like structure is fragmented among excited $0^{+}$states and the low-energy Coulomb excitation of ${ }^{10} \mathrm{Be}$ to measure the quadrupole moment of the first excited state [3], where the importance of the 3-body force is demonstrated. The neutron-rich $\mathrm{Na}$ isotopes with neutron numbers close to 20 is the ideal region to study the evolution of shell structure, where the energy gap between the neutron $s d$-pf shells undergoes significant change in a span of four neutrons from $\mathrm{N}=17$ to 20 [4]. The LLNL group led the measurement of the $\mathrm{B}(\mathrm{E} 2)$ in ${ }^{29} \mathrm{Na}(\mathrm{N}=18)$ [5] to investigate this change in shell structure off stability. Other work in the collaboration in this region included the low-energy Coulomb excitation of ${ }^{20} \mathrm{Na}$ [6] and ${ }^{21} \mathrm{Na}$ [7] to study their low-lying structure (a Ph.D. thesis was awarded for this work to M.A. Schumaker at U. of Guelph). Currently, we are also working on a study of the resonant states beyond the $\mathrm{p}$-threshold in ${ }^{22} \mathrm{Mg}$ using the inverse $\left(\mathrm{p}, \mathrm{p}^{\prime}\right)$ reaction with a ${ }^{21} \mathrm{Na}$ beam, where we have measured properties of the resonant states important to calculations of the production of ${ }^{22} \mathrm{Mg}$ in novae (the data analysis is being carried out at U. of York). 
The new $\mathrm{B}(\mathrm{E} 2)$ measurement in ${ }^{29} \mathrm{Na}$ is a compelling demonstration of a decreasing gap between the $s d$ and $p f$ shells leading to enhanced collectivity. Excitations in ${ }^{29} \mathrm{Na}$ were observed via a

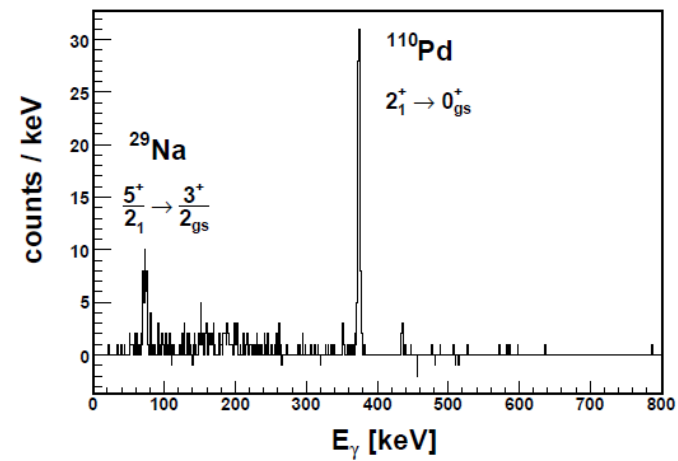

Fig. 1 : Particle- $\gamma$ coincident, suppressed, randomsubtracted $\gamma$-ray energy spectrum observed following $\sim 70 \mathrm{~h}$ of beam on target corresponding to ${ }^{110} \mathrm{Pd}\left({ }^{29} \mathrm{Na}^{29} \mathrm{Na}^{*}\right)$ at $\mathrm{E}_{\text {beam }}=70 \mathrm{MeV}$.

Transitions to ground-state are identified.

Coulomb interaction on a ${ }^{110} \mathrm{Pd}$ target as shown in Fig. 1. The ${ }^{29} \mathrm{Na}$ beam energy was well below the Coulomb barrier $\left(\mathrm{E}_{\text {beam }}=70 \mathrm{MeV}\right)$, and particlegamma coincidences were recorded using the TIGRESS array [8] together with a LLNL designed charged-particle detector array, Bambino [5]. Our measured E2 strength for the first excited state, together with the prior measured static quadrupole moment of the ground state [9], provides strong evidence for the energy-gap between neutron $s d-p f$ shells getting smaller as the "magic" number 20 is approached, indicating that the shell structure in this region is substantially different from nuclei near stability. This shell gap narrowing was well predicted by a recent calculation utilizing MonteCarlo shell-model techniques with a SPDF-M Hamiltonian [10] using a valence shell comprising all the $s d$-shell orbits and the two lowest orbits of the $p f$-shell with both in-shell and cross-shell effective interactions. We plan to continue these investigations with experiments on ${ }^{30} \mathrm{Na}$ and ${ }^{31} \mathrm{Na}$ using the SeGA array at MSU as part of a new collaboration, JANUS, or Joint Array for Nuclear Structure.

\section{Bambino2: A particle detector array for JANUS at MSU}

The deployment of the auxiliary charged-particle detectors is crucial to the success of in-beam $\gamma$ ray spectroscopy experiments using modern $4 \pi \gamma$-ray detector arrays. From a coincident measurement, one obtains not only the impact-parameter dependence of detected $\gamma$ rays but also the information needed for their Doppler-shift corrections that improve the $\gamma$-ray energy

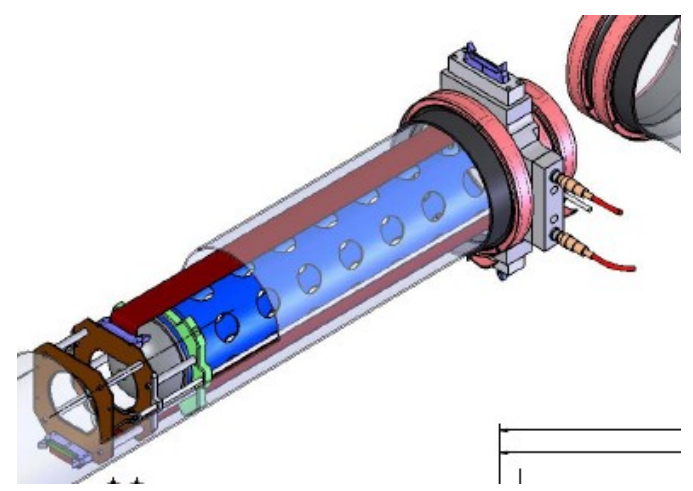

Fig. 2: Schematic diagram of LLNL's Bambino2 detector for experiments with the SeGA array. resolution thus maximizing the sensitivity of the $\gamma$-ray detector array.

For our initial work at TRIUMF, we have developed a charged-particle detector array, Bambino, for TIGRESS. The array consists of two segmented silicon detectors, each with 24 rings of $1 \mathrm{~mm}$ width and 32 sectors covering $2 \pi$. This setup has been successfully employed for six approved experiments since 2006.

For our new collaboration at MSU, JANUS, we have finalized the design of the target/detector chamber to reconcile the SeGA array in a barrel pattern (see Fig. 2 ). The fabrication of this chamber is currently being undertaken at MSU. LLNL is leading the development of a Bambino-like charged-particle detector array and its associated amplifiers. The detectors and amplifiers have been ordered and received. All the necessary tests will be completed by the end of FY11 and the detector system 
will be delivered to MSU in early FY12. The SeGA/Bambino2 setup will be ready to field experiments by early 2012 .

\section{Probing collectivity in heavy-ion reactions with particle-gamma coincidences}

In addition to the activities at the radioactive beam facility at TRIUMF, we also engaged in experiments using the stable beam facility at ANL to study 1) the population mechanism for the isomer bands in ${ }^{178} \mathrm{Hf}$ and 2 ) the role of $j_{15 / 2}$ neutrons in the rotational alignments in ${ }^{235} \mathrm{~Np}$. The latter was led by LLNL and the results have been published [11]. These experiments utilized Gammasphere with $\mathrm{CHICO}$ and were intended to investigate collective effects at high angular momentum.

The Coriolis effect in rotating nuclei leads to the mixing of the $K$ quantum number; the projected angular momentum on the nuclear symmetry axis. This leads to the band mixing of higher-spin members with very different $K$ quantum numbers, which, we believe is the pathway to population of the isomer bands in ${ }^{178} \mathrm{Hf}$. Our current effort is directed towards searching for direct transitions between the ground-state band and isomer bands among the higher-spin members to confirm the interpretation for the population mechanism of isomer bands. The data analysis is being performed at U. of Rochester.

Rotational alignment of high- $j$ orbitals such as the $i_{13 / 2}$ in the rare-earth region and $j_{15 / 2}$ in the actinides is believed to be the mechanism for observed band cross crossing phenomena in nuclei. The evidence for the rotational alignment of the $i_{13 / 2}$ neutron orbitals is firmly established but not for the $j_{15 / 2}$ neutron orbitals in actinides. The current effort has established a possible scenario of a strong interaction between the ground-state band and the aligned $j_{15 / 2}$ band in ${ }^{235} \mathrm{~Np}$. Rotational states in the ground band in ${ }^{235} \mathrm{~Np}$ were observed for the first time in this experiment using a two neutron transfer reaction ${ }^{237} \mathrm{~Np}\left({ }^{116} \mathrm{Sn},{ }^{118} \mathrm{Sn}\right){ }^{235} \mathrm{~Np}[11]$.

\section{CHICO2: Upgrading CHICO to provide kinematic reconstruction for GRETA/GRETINA}

Nuclear $\gamma$-ray spectroscopy entered a new era when the first engineering run of GRETINA [12], the first of a new generation of $\gamma$-ray tracking arrays, was successfully carried out at LBNL in early April 2011. A parallel effort has been set up to develop the auxiliary charged-particle detector arrays with a matching position resolution to fully exploit the potential of GRETINA. Improving the position resolution of the existing charged-particle detector array, CHICO [13], is a part of this coordinated effort.

CHICO has proven to be a valuable auxiliary charged-particle detector array for Gammasphere [14]. It is a highly segmented parallel-plate avalanche counter with position resolution of $\sim 1^{\circ}$ in $\theta$ and $\sim 9^{\circ}$ in $\phi$ and solid-angle coverage of $69 \%$ of $4 \pi$. Over the period between 1996 and 2008, a total of 26 experiments were fielded successfully, involving 58 experimentalists from 17 institutions; resulting in 37 publications and $5 \mathrm{Ph}$.D. theses. The proposal to upgrade $\mathrm{CHICO}$ was approved by DOE/SC at the end of FY10. The plan is to redesign the cathode board and amplifier to improve the resolution for both $\theta$ and $\phi$ coordinates to better than one degree to match that of GRETINA. This project is scheduled for completion in the summer of 2012. 
A number of tests on the pixelation pattern for the position sensing have been made at LLNL in a search for the optimal design, which was described in Ref. [15]. We have finalized the design for position-sensing cathode board by adopting full strips for $\theta$ and pixels for $\phi$. This hybrid design will preserve the $\theta$ resolution achieved in $\mathrm{CHICO}$ and significantly improve the $\phi$ resolution over that of CHICO. The redesign work on the high-gain and high-bandwidth amplifier is in progress. All of the hardware fabrication will be completed by the end of 2011 .

[1] Examples: A) C.Y. Wu, D. Cline, T. Czosnyka, A. Backlin, C. Baktash, R.M. Diamond, G.D. Dracoulis, L. Hasselgren, H. Kluge, B. Kotlinski, J.R. Leigh, J.O. Newton, W.R. Phillips, S.H. Sie, J. Srebrny, F.S. Stephens, Nucl. Phys. A 607, 178 (1996). B) C.Y. Wu, D. Cline, M.W. Simon, G.A. Davis, R. Teng, A.O. Macchiavelli, and K. Vetter, Phys. Rev. C 64, 064317 (2001), C) A.B. Hayes, D. Cline, K.J. Moody, I. Ragnarsson, C.Y. Wu, J.A. Becker, M.P. Carpenter, J.J. Carroll, D. Gohlke, J.P. Greene, A.A. Hecht, R.V.F. Janssens, S.A. Karamian, T. Lauritsen, C.J. Lister, R.A. Macri, R. Propri, D. Seweryniak, X. Wang, R. Wheeler, and S. Zhu, Phys. Rec. C 82, 044319 (2010).

[2] R. Kanungo et al., Phys. Lett. B 682, 391 (2010).

[3] N. Orce et al., submitted to Phys. Lett. B (2011).

[4] Y. Utsuno, T. Otsuka, T. Mizusaki, and M. Honma, Phys. Rev. C 60, 054315 (1999).

[5] A.M. Hurst, C.Y. Wu, et al., Phys. Lett. B 674, 168 (2009).

[6] M.A. Schumaker et al., Phys. Rev. C 80, 044325 (2009).

[7] M.A. Schumaker et al., Phys. Rev. C 78, 044321 (2008).

[8] H.C. Scraggs et al., Nucl. Instrum. Methods Phys. Res., Sect. A 543, 431 (2005).

[9] M. Keim et al., Eur. Phys. J. A 8, 31 (2000).

[10] Y. Utsuno et al., Phys. Rev. C 70, 044307 (2004).

[11] A.M. Hurst, C.Y. Wu et al., Phys. Rev. C 81, 014312 (2010).

[12] http://grfs 1.lbl.gov

[13] M.W. Simon, D. Cline, C.Y. Wu, R.W. Gray, R. Teng, and C. long, Nucl. Instrum. Methods in Phys. Res. A452, 205 (2000).

[14] I.Y. Lee, Proceedings of the Workshop on Gammasphere Physics, Berkeley, CA, Edited by M.A. Deleplanque, I.Y. Lee, and A.O. Macchiavelli (World Scientific, Singapore, 1996), p.50.

[15] C.Y. Wu, D. Cline, E. Kwan, A. Chyzh, A. Hayes, I.Y. Lee, and D. Swan, LLNL-TR484110 (2011).

\section{Search for neutrinoless double-beta decay}

Many of the biggest questions in particle and nuclear physics (as underlined by DOE/NSF Nuclear Science Advisory Committee) revolve around our limited knowledge of the properties of the elusive subatomic particle, the neutrino. Determining the neutrino mass and the relationship between the neutrino and antineutrino would provide an explanation for the grand scientific and philosophical question of how there came about a slight excess of matter over antimatter that makes up all the matter we observe in the universe today. Neutrinoless double beta decay is a long sought-after nuclear transition in which two neutrons in a nucleus are converted into two protons and two beta particles without the emission of accompanying neutrinos (see Fig. 3). A discovery would have far-reaching scientific consequences - it would determine not only the neutrino mass and hierarchy but also whether the neutrino is its own antiparticle and therefore 
whether nature violates the conservation of total lepton number. Lepton-number violation is thought to hold the key to explaining the predominance of matter over antimatter in the universe.
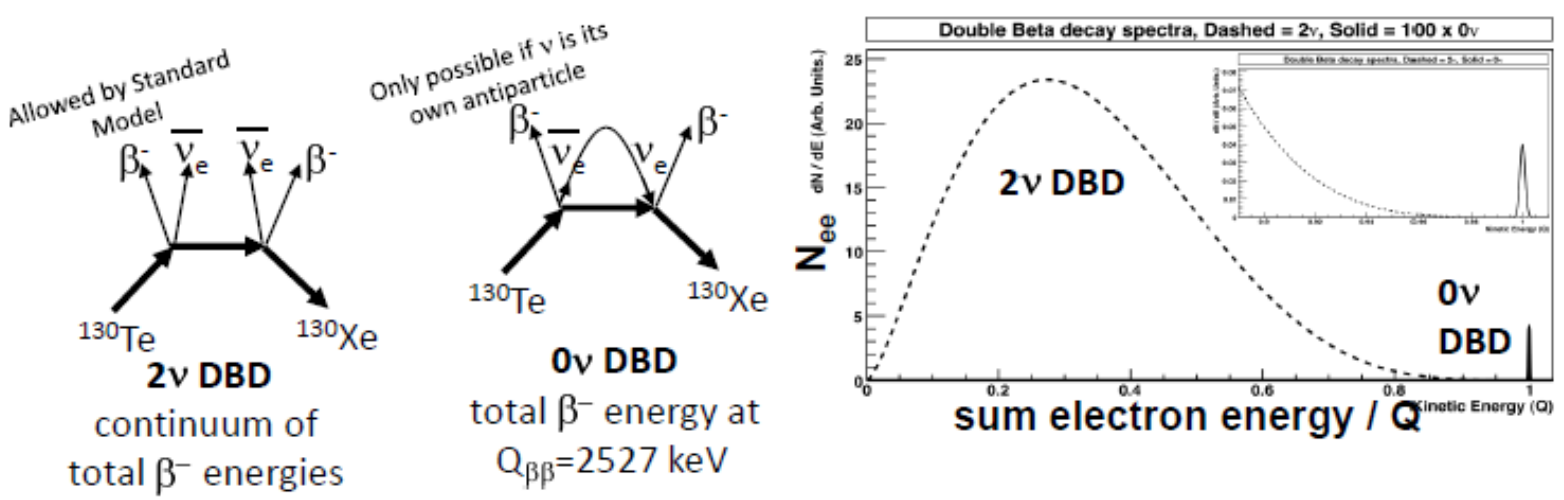

Fig. 3: On the left, the two neutrino double-beta decay mode and the neutrinoless double beta decay mode are shown. The corresponding energy spectrum of the two emitted electrons is shown on the right. For the neutrinoless case, the electrons carry away essentially all of the decay energy and a signal at the $\mathrm{Q}$ value is expected.

The Cryogenic Underground Observatory for Rare Events (CUORE) experiment brings together about 100 American and European scientists to build and operate an array of 988 cryogenic $\mathrm{TeO}_{2}$ bolometers. This will be the largest ( $\sim$ ton) cryogenic bolometer array ever realized and it will be used to perform the most sensitive search for the neutrinoless double beta $(0 v \beta \beta)$ decay of ${ }^{130} \mathrm{Te}$. Beginning in FY08, the LLNL CUORE group has taken on a significant role in the CUORE collaboration using funding from the Office of Science ( $\$ 73 \mathrm{k}$ in FY08) and LDRD (\$200k in FY08). The research performed by the LLNL CUORE group over the past four years has been crucial to improve the sensitivity of upcoming ${ }^{130}$ Te double-beta decay searches by limiting the sources of backgrounds in experiments that use $\mathrm{TeO}_{2}$ bolometers, improving the performance of the $\mathrm{TeO}_{2}$ bolometers, and precisely determining where to expect the neutrinoless double-beta decay $(0 v \beta \beta)$ signatures. This work is crucial for exploiting the scientific opportunities afforded by CUORICINO and CUORE.

In 2008, the LLNL group compared crystals produced by two vendors of $\mathrm{TeO}_{2}$ crystals, the Shanghai Institute of Ceramics, Chinese Academy of Sciences (SICCAS) and Crystal Technology, Incorporated (CTI) located in Palo Alto, CA and determined that SICCAS produced the highest radiopurity crystals. Since then, the group has been overseeing the US production of high-quality $\mathrm{TeO}_{2}$ crystal bolometers at SICCAS. The crystals represent the single largest US procurement for the project. The techniques required to meet the stringent performance and radiopurity requirements for the CUORE experiment were developed and carried out by LLNL in collaboration with the Italian colleagues [1]. In order to minimize the contamination of the crystal bulk, raw materials, reagents, and ancillary materials involved in the crystal production were monitored for radiopurity using a variety of techniques - inductively-coupled plasma mass spectrometry (ICPMS), $\gamma$-ray spectroscopy using high-purity germanium (HPGe) detectors, and $\alpha$ particle spectroscopy using surface barrier detectors. Special procedures for etching and polishing the crystal surfaces, and handling and packaging the produced crystals were developed to minimize the surface contamination of the crystals. Cryogenic tests performed on $5 \%$ of the delivered crystals have verified that the crystals perform well as bolometers with excellent 
energy resolutions ( $\sim 5 \mathrm{keV} \mathrm{FWHM}$ at the $2.5 \mathrm{MeV}$ energy of interest) and have exceeded the radiopurity requirements of the CUORE experiment [2]. The LLNL group has overseen the production of the crystals that will be used for CUORE-0 and is currently in the middle of the production of the remaining US crystals required for the full CUORE array.

As of 2008, the double-beta decay $Q$ values for ${ }^{130} \mathrm{Te},{ }^{128} \mathrm{Te}$, and ${ }^{120} \mathrm{Te}$ recommended by the 2003 Atomic Mass Evaluation had uncertainties that were larger than the resolution of the $\mathrm{TeO}_{2}$ bolometric detectors. To precisely determine where to expect the $0 v \beta \beta$ signatures, these $Q$ values were determined by a LLNL-led team that used the Canadian Penning Trap Mass Spectrometer at Argonne National Laboratory [3]. The ${ }^{130} \mathrm{Te} Q$ value was determined to be $3 \mathrm{keV}$ lower than the previously recommended value and the ${ }^{120} \mathrm{Te} Q$ value uncertainty was reduced by nearly an order of magnitude. These results have already had an impact on searches for ${ }^{130} \mathrm{Te} 0 v \beta \beta$ decay [4] and ${ }^{120}$ Te electron capture with $\beta^{+}$emission [5] using CUORICINO data. CUORICINO was the first tower array of $62 \mathrm{TeO}_{2}$ bolometers with a total mass of $40 \mathrm{~kg}$. The FY11 results for the

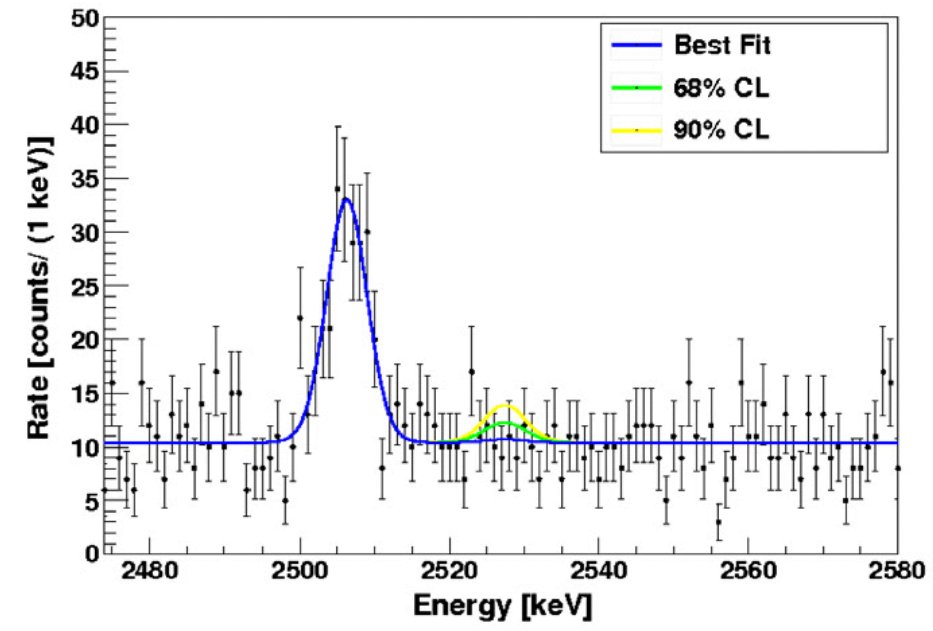

Fig. 4: Final CUORICINO results for the search for the $0 v \beta \beta$ decay of ${ }^{130} \mathrm{Te}$ [3]. The updated $\mathrm{Q}$ value is used to search for a peak in the energy spectrum. The best fit and the $68 \%$ and $90 \%$ confidence intervals are shown. The peak at $2505 \mathrm{keV}$ is due to background from ${ }^{60} \mathrm{Co}$. This analysis resulted in a halflife limit of $\mathrm{T}_{1 / 2}(0 v \beta \beta)>2.8 \times 10^{24} \mathrm{y}(90 \%$ C.L. $)[4]$. search for the ${ }^{130}$ Te $0 v \beta \beta$ decay using the updated $Q$ value is shown in Fig. 4. In addition, the fact that the $Q$ value results demonstrated that the ${ }^{120} \mathrm{Te}$ signature fortuitously is expected to lie between the ${ }^{214} \mathrm{Bi}$ lines commonly observed in lowbackground experiments was a crucial component of the analysis that improved the limits for this isotope by 3-4 orders of magnitude.

The LLNL group has also had a major role in understanding the backgrounds produced by cosmic rays. A recipient of the highlycompetitive SEGRF fellowship (a LLNL-funded appointment for top $\mathrm{Ph}$.D. students to conduct research at the laboratory, now called "Lawrence Scholarship"), Michelle Dolinski conducted her thesis research with the LLNL group to determine cross sections for neutron interactions on tellurium [6]. The $\gamma$-ray production cross sections from neutron interactions as a function of energy were measured at LANSCE at Los Alamos National Laboratory (LANL) using the GEANIE HPGe array (built in 1999 by a LLNL-LANL collaboration) to improve the simulations of neutron-induced backgrounds.

In addition, prior to the successful completion of data-taking with CUORICINO [7], large-area plastic-scintillator detectors (borrowed from the Department of Homeland Security) were placed above the CUORICINO detector to better understand the contribution of cosmic-ray muons to the CUORICINO background [8]. Although a number of additional scintillator detectors were used in this work, the detectors LLNL provided were the largest, and therefore, provided the most useful data. This work demonstrated that muon-induced backgrounds contribute negligible 
background for CUORE in the underground site at LNGS and that a muon veto will not be necessary.

In FY10, LDRD funds were leveraged to develop a system to reliably and reproducibly instrument bolometer detectors with thermistors (for signal read-out) and heater chips (for signal stabilization). Previously these pieces were glued by hand to the crystals and significant variation in the thermal connections was observed from detector to detector. The newly-developed system enables the user to place these pieces with significantly better precision, which is expected to result in more uniform performance of the bolometers. This system is now being used to instrument the CUORE-0 crystals and will be used to instrument all the CUORE crystals.

In the beginning of FY11, Dr. Marisa Pedretti, a LLNL post-doc, was enthusiastically selected to be the CUORE-0 experimental coordinator by the CUORE collaboration. This is a significant leadership role in establishing the scientific program and organizing the commissioning and operation of the CUORE-0 tower. She was selected because of her deep understanding of the CUORE experiment and the assembly and function of the components of the CUORICINO experiment as well as her extensive experience with cryogenic bolometers. The CUORE-0 tower, consisting of $52 \mathrm{TeO}_{2}$ crystal bolometers, is the first step towards the realization of CUORE. She has been stationed at the experimental site to effectively organize the onsite group of US and Italian scientific and engineering staff that is working to commission and run the CUORE-0 tower. As a result of Dr. Pedretti's efforts, there has been no major delay in the CUORE-0 schedule in the past year.

Marisa Pedretti was awarded a Physics Division SPOT award because of her leadership within the CUORE collaboration and her achievements developing novel cryogenic bolometric techniques (see for example Ref. [9]) and was featured in a LLNL Newsline article (www.llnl.gov/news/aroundthelab/2011/Jan/ATL-011011 pedretti.html) on her selection as CUORE-0 experimental coordinator. The LLNL CUORE effort was featured in LLNL's 2009 Science \& Technology Reviews (see Science \& Technology Review, June 2009, pp. 17-18 and Physical and Life Sciences 2009 Science \& Technology Review Highlights, pp. 15-16).

[1] C. Arnaboldi et al. "Production of high purity $\mathrm{TeO}_{2}$ single crystals for the study of neutrinoless double beta decay." Journal of Crystal Growth 312, 2999 (2010).

[2] F. Alessandria et al. "CUORE crystal validation runs: results on radioactive contamination and extrapolation to CUORE background", to be submitted (2011).

[3] N.D. Scielzo et al. "Double-beta decay Q values of ${ }^{130} \mathrm{Te},{ }^{128} \mathrm{Te}$, and ${ }^{120} \mathrm{Te}$ ", Physical Review C 80, 025501 (2009).

[4] E. Andreotti et al. " ${ }^{130}$ Te neutrinoless double-beta decay with CUORICINO”, Astroparticle Physics 34, 822 (2011).

[5] E. Andreotti et al. "Search for $\beta^{+} / \mathrm{EC}$ double beta decay of ${ }^{120} \mathrm{Te}$ ", Astroparticle Physics 34, 643 (2011).

[6] M.J. Dolinski. "Neutron interactions in the CUORE neutrinoless double beta decay experiment", Ph.D. Thesis, University of California, Berkeley (2008). 
[7] C. Arnaboldi et al. "Results from a search for the neutrinoless double beta decay of ${ }^{130} \mathrm{Te}$ ", Physical Review C 78, 035502 (2008).

[8] E. Andreotti et al. "Muon-induced backgrounds in the CUORICINO experiment", Astroparticle Physics 34, 18 (2010).

[9] L. Foggetta et al. "Composite macro-bolometers for the rejection of surface radioactive background in CUORE-like bolometric experiments for rare events searches", Astroparticle Physics 34, 809 (2011). 


\section{Four Year Proposed Research Plan (FY12-15)}

\section{LLNL Experimental Nuclear Physics Group}

We propose to extend prior work in the areas of collective properties of exotic nuclei and fundamental neutrino physics, and, with a modest increase in funding, initiate a new area of exploration into the physical and chemical properties of superheavy nuclei in collaboration with scientists at LBNL. This new research thrust will enable the heavy element group at LLNL to build on the discovery-class research of the last decade. This three-pronged research plan will enable three post-doctoral researchers to explore basic research in three forefront research fields: 1) to study of the evolution of nuclear shells in new regions of the chart of nuclides at the leading radioactive-ion beam facilities around the world; 2) to search for exotic decay modes, namely neutrinoless double-beta, in order to study the fundamental properties of neutrinos and test the standard model of nuclear physics; and 3) to investigate the chemical properties of heavy and superheavy nuclei in order to determine the placement of these heavy elements into the proper location in the periodic table. Each research area is described more fully in the following sections.

\section{Exploration of exotic nuclei and the collective properties of nuclei}

Activities under this task support the NSAC Subcommittee on Performance Measures report under milestone NS9 for 2018, to measure changes in shell structure and collective modes, from the most proton-rich to the most neutron-rich nuclei accessible, in order to improve our understanding of the nucleus, and to guide theory in every region of the theoretical roadmap. We propose to continue building on the work from the past four years.

Valuable experience with radioactive-beam experiments was acquired from our initial effort at TRIUMF. The upcoming low-energy radioactive beam capabilities at MSU and ANL have made it possible to explore further the evolution of shell structure in new territory of isotopes with extreme neutron/proton ratios. We propose to take advantage of this opportunity to form new collaborations to field experiments providing data important for further developing or refining the cross-shell effective interactions and our theoretical understanding of nuclear collectivity.

For the next proposed period, we will extend our research on several fronts. First, we will move to heavier nuclei and study of the evolution of shell structure in neutron-rich nuclei of $\mathrm{N} \sim 20,28$ using the ReA facility at MSU with the JANUS (Joint Array for NUclear Structure) collaboration (see Fig. 1). These experiments are a major step forward in that we will now have the capability to move further from stability and to study the collectivity of nuclear excitations in these nuclei beyond the first excited state. At ANL's CARIBU facility we will extend our exploration of the collective properties in heavy nuclei with our Gammasphere/CHICO collaboration using GRETINA/CHICO2, where the improved $\gamma$-ray energy resolution by a factor of $2-3$ allows us to carry out the experiments with low intensity of radioactive beams. 


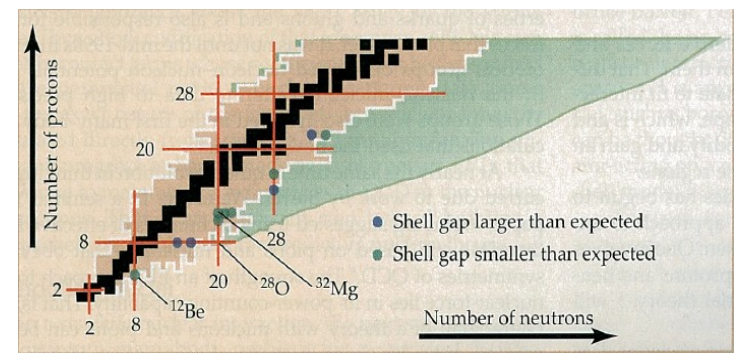

Fig 1: Portion of the chart of nuclides showing the $\mathrm{N}=20,28$ region taken from $\mathrm{D}$. Dean, Physics Today, Nov. 2007, p.48. Locations of larger and smaller than expected shell gaps are marked with blue and green dots, respectively.

In addition, we are responsible for maintaining and upgrading the auxiliary charged-particle detector arrays for both the JANUS and GRETINA/CHICO2 collaborations. Our focus at with the TIGRESS/Bambino collaboration is to further our understanding of the effect of continuum states on the collectivity of halo nuclei, e.g. ${ }^{11}$ Be. Details of our proposed research are described below.

Probing collectivity in light nuclei with particlegamma coincidences

So far, most low-energy radioactive-beam experiments measure the transition probability for the first excited state in a nucleus of interest. However, a comprehensive test of predictive power of nuclear models requires a broader set of measured quantities such as the static moment and the properties of excitations beyond the first excited state. Listed below are examples we plan to pursue in the proposed period.

\section{The evolution of shell structure in Na and S isotopes at MSU}

One neutron away from the $\mathrm{N}=20$ shell closure, ${ }^{30} \mathrm{Na}$ exhibits characteristics of enhanced collectivity [1,2] in addition to single-particle degrees of freedom. These characteristics can be attributed to the significantly reduced energy gap between $s d-p f$ shells or even shell inversion, described by calculations using Monte-Carlo shell-model techniques with a SPDF-M Hamiltonian [3]. The enhanced collectivity implies the existence of rotational or vibrational modes of motion. However, the existence and properties of the second excited state is not firmly established [2].

We propose to investigate the collectivity of ${ }^{30} \mathrm{Na}$ using the low-energy Coulomb excitation using the SeGA array at MSU, in contrast to previous measurements using intermediate-energy projectiles above the Coulomb barrier. This measurement exploits our new Bambino 2 chargedparticle array coupled to SeGA for the two-body kinematic reconstruction. This new collaboration, JANUS, includes scientists from LLNL, MSU, and the Univ. of Rochester. Our initial research focus is outlined in the Letter-of-Intent proposal [4], which was submitted to the PAC34 of MSU in Dec 2009 and given a high mark by the PAC committee.

In our long-term planning, we keep an eye on ${ }^{31} \mathrm{Na}$, where the shell closure at neutron number of 20 is totally absent resulting in enhanced collectivity that can be investigated using low-energy Coulomb excitation. This will provide the needed data to further test the predictive power of models, specifically with the cross-shell effective interactions involved.

The second example is to measure the static quadrupole moment of the first excited state for nuclei near ${ }^{44} \mathrm{~S}$ : The static moment for a given state in nuclei is very sensitive to the orbitals occupied by the valence nucleons. For neutron-rich nuclei in the vicinity of ${ }^{44} \mathrm{~S}$, the static quadrupole moment besides the transition probability thus provides another avenue to explore issues related to the evolution of shell structure due to the weakened $\mathrm{N}=28$ shell gap which 
leads to the occupation of intruder orbitals or mixed configurations. The latter is very sensitive to the cross-shell effective interactions.

The reorientation effect in the low-energy Coulomb excitation offers the opportunity to deduce the static quadrupole moment of the first excited state in these nuclei. We are going to propose these measurements once the ReA facility at MSU is ready to deliver the beam as stated in our Letter-of-Intent proposal [4].

\section{Completing Bambino2: A particle detector array for JANUS at MSU}

To accomplish our proposed program at MSU, an auxiliary charged-particle detector array is needed for the SeGA array for the particle-gamma coincidence experiments. LLNL leads the development of this auxiliary detector array based on a pair of double-sided segmented silicon detectors similar to those we developed for TIGRESS. This detector array, Bambino2, will be completed by the end of FY11 and delivered to MSU to integrate into the target/detector chamber, which is under construction at MSU. This integration will be done by early 2012 .

\section{Understanding enhanced E1 strength in halo nuclei at TRIUMF}

Besides the new initiative discussed above, we propose to analyze and publish the results for the low-energy Coulomb excitation experiment on ${ }^{11} \mathrm{Be}$, completed in FY11 at TRIUMF using TIGRESS/Bambino. The significance of this study on the development of models beyond the shell model is described in details below. After this measurement, we will investigate additional cases to improve our understanding of the role of continuum states in shell model physics.

The parity inversion and the strongest E1 strength between bound states in nuclei in ${ }^{11} \mathrm{Be}$ are difficult to interpret under the standard framework of shell model. The calculation failed to reach the convergence using the sophisticated ab initio no-core shell model with the basis up to $9 \hbar \omega$ [5]. It is doubtful for the viability of this approach to ever describe the properties of halo nuclei. Therefore, a hybrid model, ab initio no-core shell model + resonating-group method, is being developed to describe the properties of halo nuclei not only for bound sates but also for the continuum (unbound scattering states) in a unified manner [6].

The comparison of the E1 strength between measurement and calculation is one of the best ways to test the predictability of this hybrid model since the enhancement is very sensitive to the detailed wavefunction. To make this comparison possible, we have proposed to make precision measurement of the E1 strengths to both bound state and continuum concurrently in ${ }^{11} \mathrm{Be}$ using the low-energy Coulomb excitation. The proposal has been approved and the experiment is scheduled from July 27 to August 10, 2011 using the TIGRESS/Bambino setup at TRIUMF.

The E1 strength between bound states in ${ }^{11}$ Be has been measured using the Doppler-shift attenuation method [7,8] and the intermediate-energy Coulomb excitation [9,10,11]. The former has an uncertainty more than $10 \%$ and the latter has the unknown uncertainty for the reaction mechanism. Separately, the E1 strength to the continuum has been measured using the projectile breakup reaction at intermediate energy $[12,13,14,15]$. The measured E1 strength most likely is contaminated by the ${ }^{10} \mathrm{Be}$ core excitation. For our proposed experiment, we intend to measure the E1 strengths to both the bound state and continuum using the low-energy Coulomb excitation by quantifying the effect on the population of bound excited state due to the E1 coupling of the 
ground state to the continuum. This technique avoids all the uncertainty associated with the intermediate-energy experiments and at the same time provides a precision measurement.

The extended wavefunction for the ground state of ${ }^{11} \mathrm{Be}$ complicates the analysis; that is, the determination of the E1 strengths using the technique developed for the stable nuclei [16] is inappropriate. Instead, an extended continuum discretized coupled channels (XCDCC) method $[11,17]$, where the extended wavefunction is treated properly, will be used to extract the E1 strengths in ${ }^{11} \mathrm{Be}$. This analysis will be carried out in FY12 once the experiment is completed in FY11.

\section{Probing collectivity in heavy nuclei with particle-gamma coincidences}

With the CARIBU facility at ANL available soon to the user, we are going to explore the collect properties of heavy neutron-rich nuclei, in particular, the aspects of the octupole collectivity using Gammasphere/CHICO or GRETINA/CHICO2 with our existing Gammasphere/CHICO collaboration. Examples of our research focus are described below in details.

The dominant collective mode of motion exhibited in nuclei is resulting from reflectionsymmetric shapes that arise from the quadrupole degree of freedom. However, nuclei with the proton number near $34,56,88$, and the neutron number near $34,56,88,134$, can assume reflectasymmetric shapes that arise from the octupole degree freedom [18]. The even-odd staggering of the positive and negative parity yrast bands in even-even nuclei, parity doublets in odd mass nuclei, and the enhanced E1 strength due to a displacement between the center of charge and mass are among the identifiable phenomena associate with the reflect-asymmetric shapes. Nevertheless, the only observable that provides the unambiguous evidence for the enhanced octupole collectivity is the measurement of E3 strength since the enhanced E1 is very sensitive to the shell corrections [19].

The neutron-rich $\mathrm{Ba}$ isotopes $(\mathrm{Z}=56, \mathrm{~N} \sim 88)$ are among the ideal candidates to study the octupole collectivity in nuclei and can easily be accessed using the spontaneous fission sources $[20,21]$. The characteristics of octupole collectivity has been observed in ${ }^{144} \mathrm{Ba}$ and ${ }^{146} \mathrm{Ba}$ from the measured even-odd staggering of positive and negative parity bands, however, the measured $\mathrm{E} 1$ in ${ }^{146} \mathrm{Ba}$ is an order of magnitude lower than that in ${ }^{144} \mathrm{Ba}$. The non-enhanced $\mathrm{E} 1$ in ${ }^{146} \mathrm{Ba}$ is attributed to significant shell corrections [19]. We propose to field a low-energy projectile Coulomb excitation experiment for both $\mathrm{Ba}$ isotopes to measure their E3 strengths at the CARIBU facility of ANL. This will provide direct evidence of enhanced octupole collectivity in the Ba isotopes. We will carry out the proposed experiments with our established collaboration at ANL using Gammasphere together with CHICO or GRETINA together with CHICO2 when available.

In addition to the opportunity provided by the CARIBU facility at ANL, we also are engaged in a collaboration at CERN/ISOLDE to explore the octupole collectivity in $\mathrm{Rn}$ and Ra isotopes with mass near 220. We recently completed the measurement of ${ }^{224} \mathrm{Ra}$ and ${ }^{220} \mathrm{Rn}$ using the low-energy projectile Coulomb excitation with the MINIBALL [22] and a double-side segmented silicon detector. These measurements are important in the search for permanent atomic electric-dipole moments [23]. The data analysis of these measurements is being carried out at U. of Liverpool. We also have a proposal to explore the octupole collectivity for odd-A nuclei in the same mass region. 


\section{Completing CHICO2 to provide kinematic reconstruction for GRETA/GRETINA}

$\mathrm{CHICO} 2$ will be a major auxiliary charged-particle detector array with a matching position resolution for GRETINA. This upgrade, described elsewhere in the proposal, is a part of coordinated parallel effort to provide the capability of the particle-gamma coincidence experiments for GRETINA. LLNL leads the upgrade effort. We have finalized the positionsensing design with a hybrid pixelation. The fabrication of the position-sensing board and amplifier will be completed by the end of 2011. The assembling and testing will begin in early 2012 and last for about $6-8$ months. CHICO2 should be ready for GRETINA by the summer of 2012.

\section{Summary and Deliverables}

Our initial effort to study the evolution of shell structure using the radioactive beam facility at TRIUMF has been successfully carried out in the past few years. The success has encouraged us to expand our program to the soon available low-energy radioactive beam facilities at MSU and ANL, where a new territory of nuclei with extreme neutron/proton ratios will be available to further explore issues related to the evolution of shell structure. We anticipate great success in this new effort to provide the data to test and improve the predictive power of models. Our collaboration with many intuitions is the key to the success to our relatively small group at LLNL and they provide the critically needed equipment and manpower to complete the required measurements in experiments. In the meantime, we contribute our expertise to design and fabricate the auxiliary charged-particle detector array for measuring the needed two-body kinematic for observed $\gamma$ rays.

The yearly deliverables for this research effort are:

FY12: Two charged-particle detector arrays, Bambino2 and CHICO2, will be ready for SeGA at MSU and GRETINA, respectively. We will complete the analysis and publish the results on the E1 strength for ${ }^{11} \mathrm{Be}$, which is important for the development of models beyond the shell model. New initiatives on the excitation beyond the first excited states for neutron-rich nuclei with $\mathrm{N} \sim 20,28$ to further address issues related to the evolution of shell structure will be begun.

FY13: New initiatives on the excitation beyond the first excited states for neutron-rich nuclei with $\mathrm{N} \sim 20,28$ to further address issues related to the evolution of shell structure will be continued - for example the measurement of excitation beyond the first excited state in ${ }^{30} \mathrm{Na}$. Depending upon CARIBU schedule, a measurement to firmly establish octupole collectivity in neutron-rich $\mathrm{Ba}$ isotopes will be fielded.

FY14: Depending upon results of previous years work, explorations into collectivity and shell structure of neutron-rich fission products will continue - for example the static quadrupole moment near ${ }^{44} \mathrm{~S}$. Investigations of other isotopes near $\mathrm{N} \sim 20,28$ or even heavier are planned. Depending upon CARIBU schedule, the measurement to explore octupole collectivity in neutron-rich $\mathrm{Ba}$ isotopes will be continued/finished. Begin work on odd-A nuclei near $\mathrm{A}=220$ at CERN/ISOLDE.

FY15: Explorations into collectivity and shell structure of neutron-rich fission products will continue and investigations of other isotopes near $\mathrm{N} \sim 20,28$ or even heavier are planned. 
Depending upon CERN/ISOLDE results, finish octupole collectivity studies of odd-A nuclei near $\mathrm{A}=220$.

[1] B.V. Pritychenko et al., Phys. Rev. C 66, 024325 (2002).

[2] S. Ettenauer et al., Phys. Rev. C 78, 017302 (2008).

[3] Y. Utsuno, T. Otsuka, T. Mizusaki, and M. Honma, Phys. Rev. C 60, 054315 (1999).

[4] Letter of Intent for JANUS collaboration: A. Gade, C.M. Campbell, G.F. Grinyer, D. Weisshaar, D. Cline, A.B. Hayes, C.Y. Wu, M.A. Stoyer, and J. A. Becker, Dec 2009, LLNLPROP-420829.

[5] C. Forssen, , P. Navratil, W.E. Ormand, and E. Cauier, Phys. Rev. C 71, 044312 (2005).

[6] S. Quaglioni and P. Navratil, Phys. Rev. Lett. 101, 092501 (2008).

[7] S.S. Hanna, K. Nagatani, W.R. Harris, and J.W. Olness, Phys. Rev. C 3, 2198 (1971).

[8] D.J. Millener, J.W. Olness, E.K. Warburton, and S.S. Hanna, Phys. Rev. C 28, 497 (1983).

[9] M. Fauerbach et al., Phys. Rev. C 56, R1 (1997).

[10] T. Nakamura et al., Phys. Lett. B 394, 11 (1997).

[11] N.C. Summer et al., Phys. Lett. B 650, 124 (2007).

[12] T. Nakamura et al., Phys. Lett. B 331, 296 (1994).

[13] F.M. Nunes, I.J. Thompson, and R.C. Johnson, Nucl. Phys. A 596, 171 (1996).

[14] R. Palit et al., Phys. Rev. C 68, 034318 (2003).

[15] N. Fukuda et al., Phys. Rev. C 70, 054606 (2004).

[16] D. Cline, Ann. Rev. Nucl. Part. Sci. 36, 683 (1986).

[17] N.C. Summers, F.M. Nunes, and I.J. Thompson, Phys. Rev. C 74, 014606 (2006); Phys. Rev. C 73, 031603(R) (2006).

[18] P.A. Butler and W. Nazarewicz, Rev. Mod. Phys. 68, No 2, 349 (1996).

[19] P.A. Butler and W. Nazarewicz, Nucl. Phys. A533, 249 (1991).

[20] W.R. Phillips et al., Phys. Rev. Lett. 57, 3257 (1986).

[21] J.H. Hamilton et al., Prog. Nucl. Part. Phys. 35, 635 (1995).

[22] N. Warr et al., Eur. Phys. J. 20, 65 (2004).

[23] J. Dobaczewski and J. Engel, Phys. Rev. Lett. 94, 232502 (2005).

Requested FY12 budget is $\$ 250 \mathrm{~K}$ (an increase of $\$ 50 \mathrm{~K}$ to support Ching-Yen at $20 \%$ level and some small amount for travel).

\section{Exploration of fundamental neutrino physics}

Activities under this task support the NSAC Subcommittee on Performance Measures report under milestone FI14 for 2017, to extend the sensitivity of searches for neutrinoless double-beta decay in selected nuclei by a factor of ten in lifetime. We propose to continue building on the work from the past four years.

The Cryogenic Underground Observatory for Rare Events (CUORE) is an experiment that will search for the neutrinoless double beta decay of ${ }^{130} \mathrm{Te}$ and other rare and exotic decays using a large array of cryogenic bolometers. Observation of this decay mode would determine the neutrino mass scale (see Fig. 2) and demonstrate that neutrinos are Majorana in character (i.e. their own antiparticle). Note that the expected CUORE sensitivity, should $0 v \beta \beta$ decay not be discovered, eliminates most of the inverted neutrino mass hierarchy from consideration. 
Knowledge gained from this discovery would answer key questions regarding the nature of neutrinos and may explain why matter dominates over antimatter in the universe.

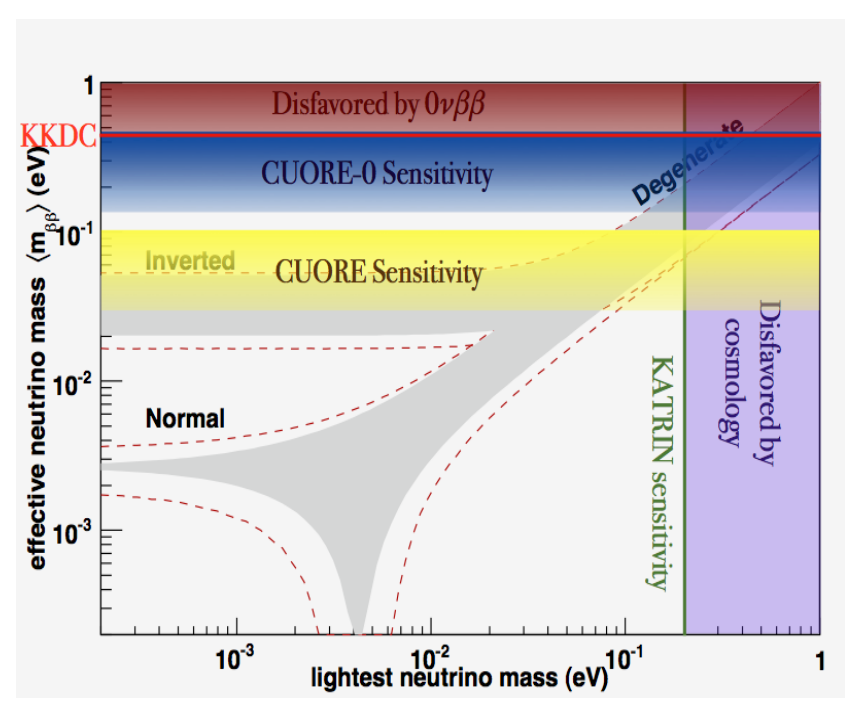

Fig 2: Neutrino mass limits achievable with CUORE-0 and CUORE. The sensitivity depends on the success of background-rejection efforts. The limits are shown as bands because of uncertainties in the nuclear matrix elements. The KKDC red-line is the published claim for discovery of $0 v \beta \beta$ decay in Ge [1]. Inverted and normal hierarchy allowed regions are shown as grey bands.
The group's efforts to characterize experimental backgrounds, understand where they originate, and determine how to best eliminate these backgrounds will continue during the CUORE Crystal Validation Runs (CCVR), the CUORE-0 scientific efforts, and the data-collection phase of the CUORE experiment. As the sensitivity of a double-beta decay experiment depends crucially on the background level around the $0 v \beta \beta$ energy region, this research is aligned well with the Low Energy Nuclear Physics program goal FI14 to improve the sensitivity of $0 v \beta \beta$ searches by a factor of ten in lifetime by 2017.

The LLNL group has significant expertise in low-background, low-energy nuclear physics and bolometers (Nicholas D. Scielzo and Marisa Pedretti along with Eric Norman from the Nuclear Engineering Department at the UC Berkeley) and a significant portion of the existing US CUORE expertise in developing sophisticated Monte Carlo simulations of the apparatus have taken research positions at LLNL (Kareem Kazkaz, Samuele Sangiorgio). In addition, Marisa Pedretti was recently enthusiastically selected by the CUORE collaboration to be the CUORE-0 experimental coordinator because of her extensive experience and expertise with cryogenic bolometers and the various components of the CUORICINO/CUORE-0/CUORE experiments. The group's efforts to characterize experimental backgrounds, understand where they originate, and determine how to best eliminate these backgrounds will be expanded during the CUORE crystal validation runs (CCVR). The group is unique in its intimate knowledge of the crystal production and therefore potential sources of contamination. In addition, two graduate students (Barbara Wang - funded through a Nuclear Forensics Fellowship from the Department of Homeland Security and a second graduate student funded by the "Nuclear Science and Security Consortium" that was recently awarded by NNSA to a UC Berkeley led consortium including LLNL) will work with the LLNL group on CUORE for their Ph.D. research.

The group will continue its years-long effort to optimize the production and delivery of highquality $\mathrm{TeO}_{2}$ crystal bolometers to meet the stringent performance and radiopurity requirements. These crystals serve as both the source and the detector for the experiment and if not produced properly could severely limit the sensitivity of the CUORE experiment by contributing the dominant radioactive background. The US CUORE crystal production will be complete by the end of FY12. 
The collaboration is enthusiastic to exploit the first tower in a stand-alone experiment and build a scientific program, outside of the CUORE construction project, that can be executed in parallel with the construction of the nineteen CUORE towers that will go on for several years. To execute the scientific program using the CUORE-0 tower there is a plan to deploy the tower array in the refurbished cryostat originally used for CUORICINO. The CUORE-0 experimental coordinator will play a significant leadership role in the scientific program of CUORE-0.

The coordination of the CUORE-0 experimental program, which began in FY11 and will continue during data-taking and analysis period, is being performed by LLNL. This effort naturally builds off the group's crystal production and background characterization efforts. The CUORE-0 scientific efforts are crucial to understand the issues that will be encountered during the construction and data-collection phase of the CUORE experiment. Once data collection begins in FY12, the CUORE-0 tower will be operated for 2 years as a stand-alone double-beta decay experiment. As a result of reduced backgrounds, this tower will surpass the sensitivity of CUORICINO within a few months. In FY12-13, the coordination of the R\&D efforts to understand and optimize the detector performance and collect data can for the most part be carried out from Livermore and therefore it is anticipated that less time will need to be spent at Gran Sasso.

As the CUORE project transitions from construction to primarily data collection, LLNL anticipates taking the lead in the US on developing detailed Monte Carlo simulations (using the GEANT4 toolkit) required to interpret the observed backgrounds. These data analysis tools will be developed and used to characterize experimental backgrounds, understand where they originate, and determine how to best eliminate these backgrounds. This work will be done in close collaboration with Italian colleagues at the University of Milan who are leading the Italian efforts. Initial efforts will be focused on determining the nature of any backgrounds observed in the $\mathrm{TeO}_{2}$ bolometers from the CCVR runs and in the CUORE-0 array. Following the insights gained from these models, a simulation of the entire CUORE array (including support structure, detector instrumentation, cryostat, and shielding) will be developed and used to understand the backgrounds.

The simulations used for background studies are also critical for much of the CUORE-0 and CUORE scientific program. LLNL will take the lead in the searches for rare decays such as the double-beta decay modes of ${ }^{120} \mathrm{Te}$ and the double-beta decay of ${ }^{130} \mathrm{Te}$ to excited states in ${ }^{130} \mathrm{Xe}$. These searches require guidance from a simulation of the detector array which can determine which decay signature provides the greatest experimental sensitivity for each decay mode. The development of the simulation model and optimization of the physics searches will begin immediately with CUORE-0 so that the tools are ready when CUORE begins collecting data. These science topics would make up a significant portion of a graduate student's Ph.D. research.

Experimental efforts to directly measure potential backgrounds will also be pursued. Neutroninduced backgrounds can be determined by irradiating sample $\mathrm{TeO}_{2}$ crystals with a neutron beam that has an energy distribution that mimics the cosmic-ray neutron energy distribution but at intensities many orders of magnitude higher. Operating a single activated crystal as a bolometer 
months and years after the irradiation can provide insight into some of the backgrounds that will be observed during the 5-year data collection period with the full 988 crystal CUORE array.

The yearly deliverables for this research effort are:

FY12: Complete production, quality control and assurance, and testing of the US portion of the $\mathrm{TeO}_{2}$ crystals. Complete CUORE-0 as a test and "shake down" of the assembly methods for CUORE. Begin data collection for the scientific program using the CUORE-0 tower. Collect data to determine long-lived isotopes resulting from cosmic-ray neutron activation.

FY13: Improve assembly line procedures based on experience from CUORE-0 and subsequent data collection with this array. Analyze data from cosmic-ray neutron activation experiment. Develop Monte Carlo simulation of CUORE-0 and CUORE to understand backgrounds and perform physics studies.

FY14: Begin data collection with CUORE array. Optimize running and operation of detector array based on experience with CUORE-0. Calibrate the CUORE Monte Carlo to data collected with the detector array and use it to understand the observed backgrounds. Using data collected from cosmic-ray neutron activation experiment and Monte Carlo simulation of CUORE, determine the expected background spectrum from cosmogenic activation in CUORE.

FY15: Apply Monte Carlo simulation to determine the efficiencies for observing various physics signatures needed for studies of ${ }^{130} \mathrm{Te}$ decays to excited states of ${ }^{130} \mathrm{Xe}$, various ${ }^{120} \mathrm{Te}$ decay modes, possible electron decay modes, and other exotic decay modes. Lead the efforts to search for these decay modes.

[1] H.V. Klapdor-Kleingrothaus et al., Nucl. Instrum. Meth. A 522, 471 (2004).

Requested FY12 budget is $\$ 400 \mathrm{~K}$ which is the level consistent with CUORE review request.

\section{Exploration of SHE physical and chemical properties}

Activities under this task support the NSAC Subcommittee on Performance Measures report under milestone NS8 for 2015, to measure properties and production mechanisms of the elements above $\mathrm{Z} \sim 102$ to understand the nature and behavior of these nuclei, and to assist theoretical predictions for the stability, structure and production of superheavy elements. We propose to begin a new program in collaboration with Rod Clark, Paul Fallon, Ken Gregorich and Heino Nitsche at LBNL to investigate the physics and chemistry of the heaviest elements. LBNL currently plans to concentrate on physics experiments with BGS + a variety of other auxiliary apparatuses such as the cube focal plane detector coupled with beta, $\mathrm{x}$-ray and gammaray detectors, the gas cell or GRETINA - experiments that would be led by LBNL that we propose to collaboratively participate in as appropriate. Experiments that would be led by LLNL would involve the testing of an automated chemistry system (see Fig. 3) using homologue aqueous chemistry and, when ready, potentially SHE (such as element 114). The automated chemistry apparatus at LLNL is being developed for nuclear forensics applications, but is easily 


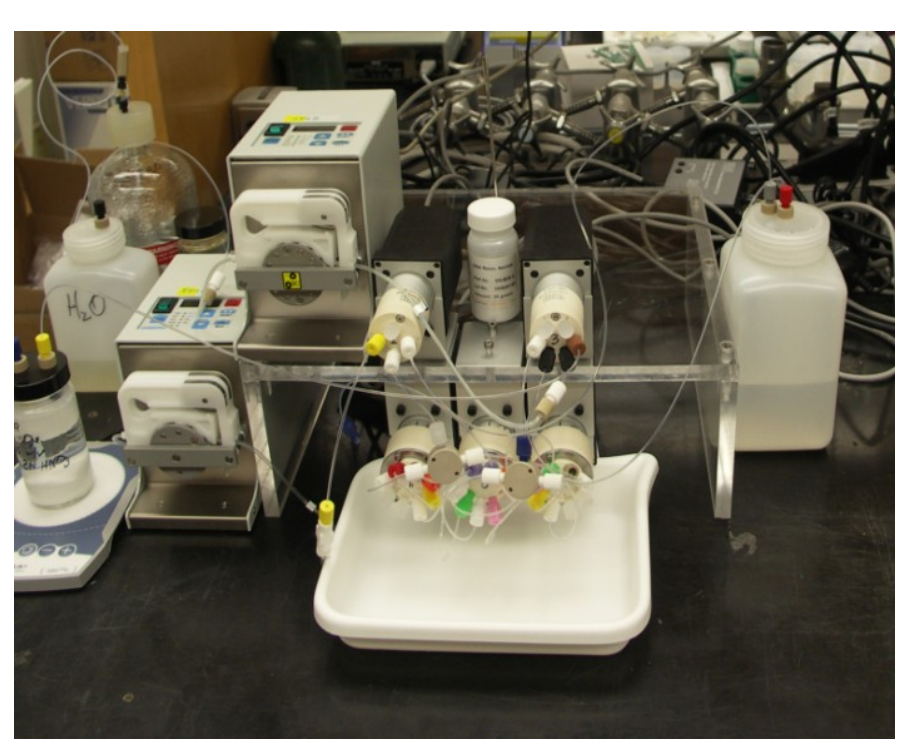

Fig 3: A photo of the LLNL automated chemistry apparatus in operation in the lab.

modified to perform atom-at-a time chemistry using micro-volumes and columns on a valve. Incidentally, this system could also be utilized for purification of isotopes reliably and reproducibly with high specific activity, a potential benefit for the isotopes program side of the DOE/SC/NP office.

State-of-the-art Dirac-Fock calculations have been performed for atoms and ions to predict the electronic shell-filling order, and hence the configuration of the periodic table for elements with $Z \leq 172$ as shown in Fig. 4 [1]. Naturally, as predictions recede from the region of the periodic table where chemical investigations of the heaviest elements have been performed $(Z \sim$ 109 , with some gas-phase investigations of $Z=112,114)$, uncertainties in the electronic shell interactions and relativistic effects make predictions less reliable. Note the predicted electronic shell filling order is interesting $\left(8 s<5 g<8 p_{1 / 2}<6 f<7 d<9 s<9 p_{1 / 2}<8 p_{3 / 2}\right)$ and the first element with $g$-orbital valence electrons is predicted to be $Z=121$, only three elements heavier than the heaviest element observed so far, element 118. It is, therefore, critical that we explore
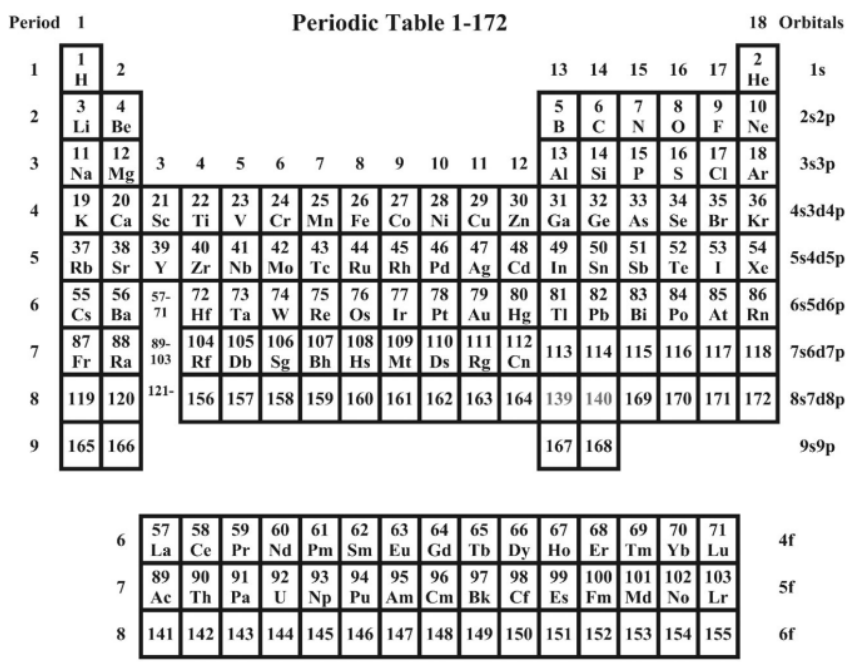

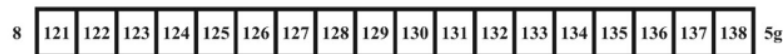

Fig 4: The extended periodic table predicted in Ref. [1]. Note that the elements do not systematically fill the table after element 138 . the chemical properties of the heaviest elements, in conjunction with production and identification of these elements via measurement of their nuclear decay properties, in order to experimentally determine the validity of such theoretical calculations, and to measure the magnitude of relativistic effects in these elements. A number of isotopes (such as $\left.{ }^{289} 114\left(t_{1 / 2}=2.6 \mathrm{~s}\right)\right)$ have been discovered with half-lives longer than a few seconds which are suitable for chemistry experiments, if a chemical separation could be reliably repeated continuously or sequentially rapidly during a long accelerator irradiation. Production of such isotopes could be enhanced utilizing thicker targets and higher beam intensities than possible in separator experiments. At LLNL, we have an interest in exploring atom-at-a- 
time aqueous chemistry, in order to elucidate the chemical behavior of these heaviest elements, which is distinct from complementary studies of gas-phased chemistry or surface chemistry. Characterization and testing of such an automated system is essential.

Thus, we specifically propose to investigate methods for putting radioactive products generated by the 88 " cyclotron into solution and by generating several isotopes with different half-lives in order to measure the shortest time such products could be processed through the system. In order to successfully perform a transactinide chemistry experiment, a transport mechanism is required to move reaction products from the target or separator to the aqueous phase of an automated chemical system. At LLNL, we are currently developing the GLITTAR (Gas-Liquid Interface for Transferring Transactinides to Automated Radiochemistry) apparatus as an interface between the Tandem accelerator at the CAMS (Center for Accelerator Mass Spectrometry) at LLNL and the SHELA (Super Heavy Element Liquid Automation) chemistry system. This type of transport interface is accelerator specific, which means that a similar system must be designed and implemented behind the BGS separator at the 88-Inch Cyclotron at LBNL for a future transactinide chemistry experiment. We require a transport mechanism from any separator system to the interface to the automated radiochemistry apparatus. Chemical experiments were carried out at the BGS the past few years [2-7] and may have already provided an appropriate interface. If not, then such an interface needs to be developed and tested. We are currently thinking of a traditional $\mathrm{He} / \mathrm{KCl}$ aerosol transport system into GLITTAR, but certainly other methods can be considered. Depending on the species to be looked at, the possibility of using a gas transport without aerosols can be considered, particularly if the species under study is predicted to have some inert character, as some predictions for the properties of element 114 indicate.

One area of constant need will be to produce appropriate homolog tracers for chemical development of separation schemes aimed at determining the properties of element 114, or any other element that has a sufficiently long-lived isotope to permit study. Current production plans at CAMS can only provide a limited set of isotopes for study. The 88 " cyclotron provides access to essentially the entire chart of the nuclides, which will allow us to produce isotopes such as ${ }^{69} \mathrm{Ge}$ and ${ }^{71} \mathrm{Ge}$ by $(\alpha, \mathrm{n})$ reactions on ${ }^{\text {nat }} \mathrm{Zn}$. Appropriate reactions for producing Group 14 elements will be investigated and developed for use at the 88-Inch Cyclotron both for off-line, bench-top batch chemical studies, and on-line efficiency measurements for system testing.

Heavy element aqueous experiments have long been plagued by the need to evaporate a final counting sample to dryness prior to successful detection of alpha radiation. We intend to counter this requirement with functionalized surface technology [8], where extractive molecules are chemically bound to a surface, currently glass, allowing species of interest to be quickly bound from an aqueous solution. The solution is removed almost immediately following contact with the substrate, and the sample is then ready for alpha counting with resolution comparable to an electroplated source. This technology permits easier access to shorter half-life species, and allows meaningful experimental statistics to be generated in shorter time periods. The extractive 
species can be tailored to the element under study, and may even offer a rapid alternative to traditional column type experiments. Fission is also an important decay mode in the super-heavy elements, and using a glass substrate will permit only single fragment measurement, whereas if the technology can be extended to a polymer surface, then the potential for being able to do fission spectroscopy on both fragments becomes possible. Developing this technology using an appropriate polymer substrate will be a key development.

The yearly deliverables for this research effort are:

FY12: Test techniques for accelerator/automated chemistry interface and measure on-line efficiency for several homologue isotopes. Utilize approximately 3 shifts of 88 " beam time. Participate in BGS+GRETINA experiments in early FY12.

FY13: Develop rapid sample preparation techniques and continue development of aqueous chemistry for group 14. May include some runs at LBL or Texas A\&M (TAMU).

FY14: Based on the results of prior tests at 88 " cyclotron, determine best location for element 114 aqueous chemistry experiment (88", Dubna, GSI, or TAMU).

FY15: Based on results from prior developments, attempt the first aqueous SHE chemistry (eg., element 114).

[1] P. Pyykkö, Phys. Chem. Chem. Phys. 13, 161 (2011).

[2] J.P. Omtvedt et al., J. Nucl. Radiochem. Sci. 3, 121 (2002).

[3] L. Stavsetra et al., Nucl. Instrum. Meth. Phys. Res. A 543, 509 (2005).

[4] R. Sudowe et al., Radiochim. Acta 94, 123 (2006).

[5] J.M. Gates et al., Radiochim. Acta 97, 167 (2009).

[6] J.M. Gates et al., Radiochim. Acta 97, 173 (2009).

[7] Ch.E. Düllmann et al., Radiochim. Acta 97, 403 (2009).

[8] W.J. Oldham et al., J Radioanal. Nucl. Chem. 282, 585 (2009).

Requested start-up budget is $\$ 150 \mathrm{~K}$ for support of 0.5-0.75 PD (institution supports 0.25), travel, and some chemical and material expenses. 


\section{LLNL institutional support}

The Experimental Nuclear Physics Group is part of the Physics Division, in the Physical and Life Sciences Directorate (PLS) of the Lawrence Livermore National Laboratory (LLNL). The Lawrence Livermore National Laboratory was established in the 1950's to design and test nuclear weapons, and nuclear physics has been an integral part of the Laboratory since its inception. With the lab's multidisciplinary structure, we have a long heritage of successfully conducting both large- and small-scale experiments, and effectively balancing research with our programmatic responsibilities.

We are located in the several buildings at the laboratory including B194 electron accelerator building, which gives us direct access to laboratory space and infrastructure built up for nuclear physics experiments at LLNL. In addition to the standard facilities supplied by LLNL such as offices, computers, and laboratories, the laboratory also supplies specialized engineering support and machine shop precision metrological capabilities. If needed, the institution also supplies super-computer machine time and specialized analytical support. We have strong connections with the stockpile stewardship radiochemistry group in the Chemistry Division within PLS which enables access to radiochemistry laboratories with both fume hoods and gloved boxes, counting rooms for measurement of emitted radiations, mass spectrometry laboratories, and both classified and unclassified work areas. We also have access to a wide range of analytical techniques including automated ion-chromatography, X-ray fluorescence, organic gas chromatography, mass spectroscopy, detector development laboratories including gamma-ray, $\mathrm{x}$-ray and particle detectors of varying types, the TPC laboratory, etc.

Our research projects dictate that we participate in experiments at accelerator and reactor facilities around the world. We have collaborations with the 88" cyclotron at the Lawrence Berkeley National Laboratory, the ATLAS accelerator at ANL, ISAC at TRIUMF, NSCL and FRIB at MSU, TUNL on the Duke Univ. campus, LANSCE at LANL, ISOLDE at CERN, JINR at Dubna, Univ. of Oslo, Univ. of Richmond, Univ. of Maryland, Univ. of Rochester, Rutgers Univ., ORNL, and are developing connections with Texas A\&M. We are currently collaborating with the Heavy Element Group at the Joint Institute for Nuclear Research in Dubna, Russia, and have the use of their facilities, including several heavy-ion cyclotrons.

Furthermore, we cooperate with the High-Flux Isotope Reactor at the Oak Ridge National Laboratory, which has provided us with an extensive inventory of exotic heavy element isotopes of varying enrichments, suitable for spectroscopic studies themselves, or as target materials for synthesis of heavier nuclear species. Our ability to make radioactive targets of exotic nuclides on thin substrates is unequalled by any other facility in the country. We have also used irradiations at HFIR to activate NIF capsules for development of radiochemistry experiments at NIF.

Through offering an exciting basic nuclear science research component, we are able to effectively recruit young scientists that are interested in nuclear science as postdoctoral researchers or graduate students. We routinely have $\sim 6$ post doctoral researchers within the group at any time, two of which are directly supported by DOE/SC/NP. We typically have 1-2 graduate students working with us at any time, mostly on DOE/SC/NP projects, but they are supported by DHS or NNSA funding through their home institution. During the summer, we typically have $\sim 6$ undergraduate researchers participating in a variety of projects in the group. 


\section{Publications during 2008-2011}

\section{Exotic nuclei studies}

1. A.B. Hayes, D. Cline, K.J. Moody, I. Ragnarsson, C.Y. Wu et al., "Coulomb excitation of a ${ }^{242} \mathrm{Am}$ isomeric target: E2 and E3 strengths, rotational alignment, and collective enhancement”, Phys. Rev. C 82, 044319 (2010).

2. A.M. Hurst, C.Y. Wu et al., "Rotational alignment in ${ }^{235} \mathrm{~Np}$ and the possible role of $j_{15 / 2}$ neutrons", Phys. Rev. C 81, 014312 (2010).

3. R. Kanungo, et al., "Structure of states in ${ }^{12} \mathrm{Be}$ via the ${ }^{11} \mathrm{Be}(\mathrm{d}, \mathrm{p})$ reaction", Phys. Lett. B 682, 391 (2010).

4. M.A. Stoyer, et al., "Nuclear physics and heavy element research at Lawrence Livermore National Laboratory”, Russian Chemical Reviews 78(12),1145 (2009).

5. M.A. Schumaker, D. Cline, G. Hackman, C.J. Pearson, C.E. Svensson, C.Y. Wu et al., "Coulomb excitation of the proton-dripline nucleus ${ }^{20} \mathrm{Na"}$, Phys. Rev. C 80, 044325 (2009).

6. A.M. Hurst, C.Y. Wu et al., "Narrowing of the neutron sd-pf shell gap in ${ }^{29} \mathrm{Na}$ ", Phys. Lett. B 674, 168 (2009).

7. U.S. Tandel, S.K. Tandel, P. Chowdhury, D. Cline, C.Y. Wu et al., "Collective oblate rotation at high spins in neutron-rich ${ }^{180}$ Hf”, Phys. Rev. Lett. 101, 182503 (2008).

8. M.A. Schumaker,D. Cline, G. Hackman, A.C. Morton, C.J. Pearson, C.E. Svensson, C.Y. Wu et al., "Coulomb excitation of radioactive ${ }^{21} \mathrm{Na}$ and its stable mirror ${ }^{21} \mathrm{Ne}$ ", Phys. Rev. C 78, 044321 (2008).

9. M. Wiedeking, et al., "Intruder excitations in ${ }^{35}$ P", Phys. Rev. C 78, 037302 (2008).

10. W.D. Kulp, et al., "Search for intrinsic collective states in ${ }^{152} \mathrm{Sm}$ ”, Phys. Rev. C 77, 061301(R) (2008).

\section{CUORE neutrinoless double-beta decay studies}

1. E. Andreotti et al. " ${ }^{130} \mathrm{Te}$ neutrinoless double-beta decay with CUORICINO”, Astroparticle Physics 34, 822 (2011).

2. L. Foggetta et al. "Composite macro-bolometers for the rejection of surface radioactive background in CUORE-like bolometric experiments for rare events searches", Astroparticle Physics 34, 809 (2011).

3. E. Andreotti et al. "Search for $\beta^{+} / \mathrm{EC}$ double beta decay of ${ }^{120} \mathrm{Te}$ ", Astroparticle Physics 34, 643 (2011).

4. C. Arnaboldi et al. "Production of high purity $\mathrm{TeO}_{2}$ single crystals for the study of neutrinoless double beta decay”, Journal of Crystal Growth 312, 2999 (2010). 
5. E. Andreotti et al. "Muon-induced backgrounds in the CUORICINO experiment", Astroparticle Physics 34, 18 (2010).

6. N.D. Scielzo et al. "Double-beta decay Q values of ${ }^{130} \mathrm{Te},{ }^{128} \mathrm{Te}$, and ${ }^{120} \mathrm{Te}$ ", Physical Review C 80, 025501 (2009).

7. M. Pedretti et al. "CUORE experiment: The search for neutrinoless double beta decay", International Journal of Modern Physics A 23, 3395 (2008).

8. M.J. Dolinski. "Neutron interactions in the CUORE neutrinoless double beta decay experiment", Ph.D. Thesis, University of California, Berkeley (2008).

9. C. Arnaboldi et al. "Results from a search for the neutrinoless double beta decay of ${ }^{130}$ Te", Physical Review C 78, 035502 (2008).

In addition, a number of papers are nearly ready to be submitted:

1. E. Andreotti et al. "Study of the double-beta decay of ${ }^{130} \mathrm{Te}$ to the first $0^{+}$excited state of ${ }^{130} \mathrm{Xe}$ with the CUORICINO detector", to be submitted (2011).

2. F. Alessandria et al. "Observation of ${ }^{123} \mathrm{Te}$ electron capture decay from the $\mathrm{L}$ atomic shell", to be submitted (2011).

3. E. Andreotti et al. "CUORICINO: construction and operation", to be submitted (2011).

4. F. Alessandria et al. "Sensitivity of CUORE to neutrinoless double-beta decay", to be submitted (2011).

5. F. Alessandria et al. "CUORE crystal validation runs: results on radioactive contamination and extrapolation to CUORE background", to be submitted (2011).

Awards:

M. Pedretti - SPOT award from the Physics Division of the Physical and Life Sciences Directorate, LLNL (2011) for her leadership within the CUORE collaboration.

Recognitions

M. Pedretti - selected as CUORE-0 experimental coordinator (2010).

M. Pedretti - featured in a LLNL Newsline article (www.llnl.gov/news/aroundthelab/2011/Jan/ATL-011011_pedretti.html) for her selection as CUORE-0 experimental coordinator (2010).

N.D. Scielzo - featured in LLNL's Science \& Technology Reviews (see Science \& Technology Review, June 2009, pp. 17-18 and Physical and Life Sciences 2009 Science \& Technology Review Highlights, pp. 15-16) for research in double-beta decay (2009). 


\section{Summary Table of Publications}

\begin{tabular}{|l|c|c|c|c|c|c|}
\hline \multicolumn{1}{|c|}{ Scientist } & $\begin{array}{c}\text { No. career } \\
\text { papers* }\end{array}$ & $\begin{array}{c}\text { No. papers } \\
2008-2011^{*}\end{array}$ & $\begin{array}{c}\mathrm{H} \\
\text { index* }\end{array}$ & $\begin{array}{c}\text { No. papers } \\
1^{\text {st }} \text { author }\end{array}$ & $\begin{array}{c}\text { No. papers } \\
2008-2011 \\
\text { (lead role) }\end{array}$ & $\begin{array}{c}\text { No. papers } \\
\text { career } \\
\text { (lead role) }\end{array}$ \\
\hline Nick Scielzo & 33 & 22 & 9 & 9 & 16 & 25 \\
\hline Marisa Pedretti & 64 & 12 & 10 & 11 & 10 & 48 \\
\hline Ching-Yen Wu & 85 & 15 & 19 & 8 & 10 & 60 \\
\hline Aaron Hurst & 9 & 7 & 4 & 3 & 5 & 6 \\
\hline Elaine Kwan & 23 & 15 & 6 & 2 & 10 & 18 \\
\hline Mark Stoyer & 269 & 34 & 31 & 20 & 20 & 180 \\
\hline
\end{tabular}

*Data from ISI Web of Knowledge/Web of Science extracted July 2011. 


\section{Ching-Yen Wu}

\section{Education:}

Ph.D. in Physics, University of Rochester, (Advisor: Prof. Douglas Cline), 1983

M.A. in Physics, University of Rochester, 1979

B.S. in Physics, National Central University, Taiwan, R.O.C., 1976

\section{Academic Appointments:}

Staff Physicist, Lawrence Livermore National Laboratory; 2005 to present

Senior Research Associate, University of Rochester; 1987 to 2005

Research Associate, University of Tennessee; 1985 to 1987

Research Associate, University of Rochester; 1983 to 1985

Professional services:

Referee for Physical Review C, Physical Review Letters, and European Physical Journal A; reviewer for DOE SBIR/STTR program

\section{Community services:}

Member of Gammasphere user executive committee (2010 - 2011, 2002 - 2003)

\section{Selected Publications: (85 total in refereed journals; 15 since 2008)}

10. A.M. Hurst, C.Y. Wu et al., "Narrowing of the neutron $s d-p f$ shell gap in ${ }^{29} \mathrm{Na",} \mathrm{Phys.} \mathrm{Lett.} \mathrm{B} 674$, 168 (2009).

9. M. Jandel et al., "Neutron capture cross section of ${ }^{241}$ Am", Phys. Rev. C 78, 034609 (2008).

8. A.P. Tonchev et al., "Measurement of the ${ }^{241} \mathrm{Am}(\mathrm{n}, 2 \mathrm{n})$ reaction cross section from $7.6 \mathrm{MeV}$ to 14.5 MeV", Phys. Rev. C 77, 054610 (2008).

7. A. B. Hayes, D. Cline, C. Y. Wu, et al., "Spin dependence of $K$ mixing, strong configuration mixing, and electromagnetic properties of ${ }^{178} \mathrm{Hf}$ ", Phys. Rev. C 75, 034308 (2007).

6. C.Y. Wu et al., "Evidence for possible shape transitions in neutron-rich Ru isotopes: spectroscopy of ${ }^{109,110,111,112}$ Ru", Phys. Rev. C 73, 034312 (2006).

5. A. B. Hayes, D. Cline, C. Y. Wu, et al., "Breakdown of $\mathrm{K}$ selection ${ }^{178} \mathrm{Hf}$, Phys. Rev. Lett. 96, 042505 (2006).

4. C.Y. Wu, H. Hua, and D. Cline, "The role of the intrinsic E2 matrix element between the first two 0+ states in their configuration mixing in ${ }^{100} \mathrm{Zr}$ ", Phys. Lett. B 541, 59 (2002).

3. C.Y. Wu and D. Cline, "Triaxiality in quadrupole deformed nuclei", Phys. Rev. C 54, 2356 (1996).

2. C.Y. Wu and D. Cline, "Fragmentation of two-phonon gamma-vibration strength in deformed nuclei", Phys. Lett. B382, 214 (1996).

1. C.Y. Wu, W. von Oertzen, D. Cline, and M.W. Guidry, "Pairing correlations and two-nucleon transfer between heavy nuclei”, Ann. Rev. Nucl. Part. Sci. 40, 285 (1990).

\section{Synergistic Activities:}

1. Current PI for

a. Fission-neutron measurement (WCI); responsible to develop a new generation of fission chamber to provide a fast and clean trigger for the Chi-Nu neutron detector array.

b. Evolution of shell structure in neutron-rich nuclei (DOE/SC); exploring the structure of nuclei with extreme isospin with radioactive beams.

d. Neutron capture measurement at DANCE (NA22); improving the $(n, \gamma)$ measurement for ${ }^{238,241} \mathrm{Pu}$ by developing a new fission detector system for DANCE. 
2. Particle detector: Key person in developing a) Gas avalanche fission-trigger counters as auxiliary detectors for the $(\mathrm{n}, \gamma)$ and $(\mathrm{n}, f)$ measurements with actinide targets, b) Bambino, a segmented silicon array for TIGRESS in TRIUMF, and c) CHICO2, a pixelated parallel-plate avalanche counter with a matching position resolution for GRETINA.

3. Coulomb excitation: Key role in developing both the experimental and analysis tools for the second-generation of Coulomb excitation study of collective modes of motion in nuclei.

4. Spectroscopy of neutron-rich nuclei: Pioneer role in establishing the nuclear structure of neutron-rich nuclei, produced in the fusion-fission reaction.

\section{Collaborators:}

1. Chi-Nu collaboration: R. Haight et al. (LANL)

2. DANCE collaboration: J. Ullmann et al. (LANL)

3. TIGRESS/Bambino collaboration: 52 participants from 15 institutions

4. Gammasphere/CHICO collaboration: 58 participants from 17 institutions

5. JANUS collaboration : 9 participants from 3 institutions

Postdoctoral Advisees in LLNL:

Aaron Hurst (2007 - 2009, curent location : LBNL), Andrii Chyzh (2010 - present), Elaine Kwan (2010 - present) 


\section{Elaine Kwan}

Physics Division, Physical and Life Sciences Directorate Lawrence Livermore National Laboratory

Phone: 925-424-3420

Email: kwan12@1lnl.gov

\section{Education:}

- Ph.D. in Physics (Advisor: University Distinguished Professor David J. Morrissey). Michigan State University, East Lansing, MI, United States, 12/2006.

- M.S. in Physics. Michigan State University, East Lansing, MI, United States, 2002.

- B.A. in Mathematics. University of Michigan-Dearborn, Dearborn, MI United States, 1998

- B.S. in Physics. University of Michigan-Dearborn, Dearborn, MI United States, 1998

\section{Relevant Work History:}

- Lawrence Livermore National Laboratory, Livermore, CA, United States, Postdoctoral Researcher, from $6 / 2010$ to present.

- Duke University, Durham, NC, United States, Postdoctoral Researcher, from 01/2007 to $6 / 2010$.

- Michigan State University and National Superconducting Cyclotron Laboratory, East Lansing, MI, United States, Graduate Student Researcher, from 2003 to 12/2006.

Professional Leadership and Service:

reviewer for Atomic Data and Nuclear Data Tables

\section{Three Most Important Publications:}

"Discrete deexcitations in ${ }^{235} \mathrm{U}$ below $3 \mathrm{MeV}$ from nuclear resonance fluorescence" $\mathrm{E}$. Kwan, G. Rusev, A. Adekola, F. Dönau, S. L. Hammond, C. R. Howell, H. J. Karwowski, J. H. Kelley, R. S. Pedroni, R. Raut, A.P. Tonchev, W. Tornow, Phys. Rev. C 83, 041601(R) (2011)

"Precision Mass Measurements of Rare Isotopes Near $N=Z=33$ Produced by Fast Beam Fragmentation" P. Schury, C. Bachelet, M. Block, G. Bollen, D. A. Davies, M. Facina, C. M. Folden III, C. Guénaut, J. Huikari, E. Kwan, A. Kwiatkowski, D. J. Morrissey, R. Ringle, G.K. Pang, A. Prinke, J. Savory, H. Schatz, S. Schwarz, C. S. Sumithrarachchi, T. Sun, Phys. Rev. C. 75, 055801 (2007)

"Experiments with Thermalized Rare Isotope Beams from Projectile Fragmentation: A Precision Mass Measurement of the Superallowed $\beta$ Emitter ${ }^{38} \mathrm{Ca}^{\prime \prime}$ G. Bollen, D. Davies, M. Facina, J. Huikari, E. Kwan, P. A. Lofy, D. J. Morrissey, A. Prinke, R. Ringle, J. Savory, P. Schury, S. Schwarz, C. Sumithrarachchi, T. Sun, L. Weissman, Phys. Rev. Lett. 96, 152501 (2006) 


\section{Aaron M Hurst}

Isotopes Project, Nuclear Science Division

Lawrence Berkeley National Laboratory

Phone: 510-486-6152

Email: $\underline{\text { AMHurst@,lbl.gov }}$

\section{Education:}

- Ph.D. in Nuclear Physics. University of Liverpool, Liverpool, United Kingdom, 09/2006.

- M. Sc. in Nuclear and Radiation Physics. University of Manchester, Manchester, United Kingdom, 10/2002.

- B.Sc. (Honours) in Chemistry. University of Nottingham, Nottingham, United Kingdom, 06/2000.

\section{Work History:}

- Lawrence Berkeley National Laboratory, Berkeley, CA, United States, Physicist Project Scientist, from 03/2010 to present.

- Lawrence Livermore National Laboratory, Livermore, CA, United States, Postdoctoral Fellow, from 02/2007 to 02/2010.

- University of Liverpool, Liverpool, United Kingdom, Graduate Student Researcher, from $10 / 2002$ to $09 / 2006$.

\section{Awards and Honors:}

Spot Award, Physics Division, Lawrence Livermore National Laboratory, 2009.

\section{Three Most Important Publications:}

A.M. Hurst, C.Y. Wu, J.A. Becker, M.A. Stoyer, C.J. Pearson, G. Hackman, M.A. Schumaker, C.E. Svensson, R.A.E. Austin, G.C. Ball, D. Bandyopadhyay, C.J. Barton, A.J. Boston, H.C. Boston, R. Churchman, D. Cline, S.J. Colosimo, D.S. Cross, G. Demand, M. Djongolov, T.E. Drake, P.E. Garrett, C. Gray-Jones, K.L. Green, A.N. Grint, A.B. Hayes, K.G. Leach, W.D. Kulp, G. Lee, S. Lloyd, R. Maharaj, J.-P. Martin, B.A. Millar, S. Mythili, L. Nelson, P.J. Nolan, D.C. Oxley, E. Padilla-Rodal, A.A. Phillips, M. Porter-Peden, S.V. Rigby, F. Sarazin, C.S. Sumithrarachchi, S. Triambak, P.M. Walker, S.J. Williams, J. Wong, J.L. Wood. "Narrowing of the neutron sd-pf shell gap in ${ }^{29} \mathrm{Na}$ ", Physics Letters B 674, 168 (2009).

J. Cederkall, A. Ekstrom, C. Fahlander, A.M. Hurst, M. Hjorth-Jensen, F. Ames, A. Banu, P.A. Butler, T. Davinson, U. Datta Pramanik, J. Eberth, S. Franchoo, G. Georgiev, M. Gorska, D. Habs, M. Huyse, O. Ivanov, J. Iwanicki, O. Kester, U. Koster, B.A. Marsh, O. Niedermaier, T. Nilsson, P. Reiter, H. Scheit, D. Schwalm, T. Sieber, G. Sletten, I. Stefanescu, J. Van de Walle, P. Van Duppen, N. Warr, D. Weisshaar, F. Wenander. "Sub-Barrier Coulomb Excitation of ${ }^{110}$ Sn and Its Implications for the ${ }^{100}$ Sn Shell Closure”, Physical Review Letters 98, 172501 (2007). 
A.M. Hurst, P.A. Butler, D.G. Jenkins, P. Delahaye, F. Wenander, F. Ames, C.J. Barton, T. Behrens, A. Burger, J. Cederkall, E. Clement, T. Czosnyka, T. Davinson, G. de Angelis, J. Eberth, A. Ekstrom, S. Franchoo, G. Georgiev, A. Gorgen, R.-D. Herzberg, M. Huyse, O. Ivanov, J. Iwanicki, G.D. Jones, P. Kent, U. Koster, T. Kroll, R. Krucken, A.C. Larsen, M. Nespolo, M. Pantea, E.S. Paul, M. Petri, H. Scheit, T. Sieber, S. Siem, J.F. Smith, A. Steer, I. Stefanescu, N.U.H. Syed, J. Van de Walle, P. Van Duppen, R. Wadsworth, N. Warr, D. Weisshaar, M. Zielinska. "Measurement of the Sign of the Spectroscopic Quadrupole Moment for the $2^{+}{ }_{1}$ State in ${ }^{70}$ Se: No Evidence for Oblate Shape”, Physical Review Letters 98, 072501 (2007). 


\section{Nicholas D Scielzo}

Physics Division, Physical and Life Sciences Directorate

Lawrence Livermore National Laboratory

Phone: 925-422-4172

Email: scielzo1@,1lnl.gov

\section{Education:}

- Ph.D. in Nuclear Physics. University of California, Berkeley, Berkeley, CA, United States, 05/2003.

- B.A. in Chemistry and Physics. Harvard University, Cambridge, MA, United States, 06/1997.

\section{Work History:}

- Lawrence Livermore National Laboratory, Livermore, CA, United States, Staff Physicist, from 08/2009 to present.

- Lawrence Livermore National Laboratory, Livermore, CA, United States, Lawrence Fellow, from 10/2006 to 08/2009.

- Argonne National Laboratory, Argonne, IL, United States, Postdoctoral Researcher, from $07 / 2003$ to $10 / 2006$.

- Lawrence Berkeley National Laboratory, Berkeley, CA, United States, Interim Postdoctoral Researcher, from 05/2003 to 06/2003.

- Lawrence Berkeley National Laboratory, Berkeley, CA, United States, Graduate Student Researcher, from 06/1998 to 05/2003.

\section{Awards and Honors:}

Outstanding Performance Award, Lawrence Berkeley National Laboratory, 2000. Lawrence Fellow Award, Lawrence Livermore National Laboratory, 2006.

\section{Professional Leadership and Service:}

Physical Review C, Reviewer, from 2011 to Present

Physics Letters B, Reviewer, from 2007 to Present

SIGMA, Reviewer, from 2006 to Present

\section{Three Most Important Publications:}

G. Savard, F. Buchinger, J.A. Clark, J.E. Crawford, S. Gulick, J.C. Hardy, A.A. Hecht, J.K.P. Lee, A.F. Levand, N.D. Scielzo, H. Sharma, K.S. Sharma, I. Tanihata, A.C.C. Villari, Y. Wang. " $Q$ value of the superallowed decay of $V-46$ and its influence on $V$-ud and the unitarity of the Cabibbo-Kobayashi-Maskawa matrix", Physical Review Letters 95, 102501 (2005).

N.D. Scielzo, S.J. Freedman, B.K. Fujikawa, P.A. Vetter. "Measurement of the beta-nu correlation using magneto-optically trapped Na-21", Physical Review Letters 93, (2004). 
LLNL-TR-497871

J.R. Guest, N.D. Scielzo, I. Ahmad, K. Bailey, J.P. Greene, R.J. Holt, Z.-T. Lu, T.P. O'Connor, D.H. Potterveld. "Laser trapping of Ra-225 and Ra-226 with repumping by room-temperature blackbody radiation", Physical Review Letters 98, 093001 (2007). 


\author{
Marisa Pedretti \\ Physics Division, Physical and Life Sciences Directorate \\ Lawrence Livermore National Laboratory \\ Phone: 925-424-5945 \\ Email: pedretti2@1lnl.gov
}

\title{
Education:
}

- Ph.D. Insubria University, Como, Italy, 01/2004. Thesis title: "The single module for Cuoricino and CUORE detectors: tests, construction and modelling”. http://www.roma1.infn.it/exp/cuore/thesis/phd/Pedretti phd.pdf . Advisor: A. Giuliani.

- Laurea (Italian M.S. degree). Milano University, 05/2000. Dissertation title: "Development of low temperature detector arrays for the measurement of electron antineutrino mass" (written in Italian). Advisor: E. Fiorini.

Work History:

Jan. 2009 - present: Post-doctoral Researcher at Lawrence Livermore National Laboratory.

May 2005 - Dec 2008: Post-doctoral Fellowship of Instituto Nazionale di Fisica Nucleare (INFN) at Milano-Bicocca University.

Jan. 2004 - Dec. 2004: Post-doctoral Fellowship of Insubria University.

Jun. 2001 - Aug. 2001: Research period at the Kamerlingh Onnes Laboratory (Leiden University, Netherlands) on superconductive transducers for cryogenic gravitational wave antennas.

\section{Awards and Honors:}

SPOT award - Physics Division of the Physical and Life Sciences Directorate, LLNL (2011). Short-listed in 2010 for a scientific staff position at SLAC Laboratory. Short-listed in 2009 for a tenure-track position at Columbia University.

Obtained INFN "researcher qualification" status in astroparticle physics after competing in an INFN national competition (2005).

\section{Three Most Important Publications:}

C. Arnaboldi et al. "New limit on the neutrinoless beta bb decay of 130Te", Physical Review Letters 95, 142501 (2004).

C. Arnaboldi et al. "First results on neutrinoless double beta decay of 130Te with the calorimetric CUORICINO experiment”, Physics Letters B 584, 260 (2004).

C. Arnaboldi et al. "CUORE: A cryogenic underground observatory for rare events", Nuclear Instruments and Methods in Physics Research Section A 518, 775 (2004). 


\section{Mark A. Stoyer}

\section{L-414}

Physics Division

Physical and Life Sciences Directorate

7000 East Ave.

Lawrence Livermore National Laboratory

Phone: 925-423-3079

Email: stoyer1@1lnl.gov

\section{Education:}

University of California, Berkeley, Berkeley, CA, United States, attended from 08/1985 to 11/1990, PhD, Nuclear Chemistry, 11/1990.

Purdue University, West Lafayette, IN, United States, attended from 08/1981 to 12/1984, B.S. in Chemistry, 12/1984.

\section{Work History:}

1/85 - 8/85 Research Assistant at LLNL, Nuclear Chemistry Division, with Dr. Richard Meyer 8/85 - 11/90 Grad. Student Research Assistant at LBL with Dr. John Rasmussen 11/90 - 2/91 Post Doctoral Research Assistant at LBL with Dr. John Rasmussen 2/91 - 8/93 Post-Doctoral Research Assistant at LLNL with Dr. Eugene Henry 8/93 - 6/95 Post-Doctoral Research Assistant at LBL with Dr. Michael Nitschke 6/95 - 2/07 Staff Scientist, Stockpile Stewardship Radiochemistry Group, LLNL 6/01 - 9/03 Deputy Program Element Leader, Stockpile Stewardship Radiochem Group, LLNL 6/02 - 7/03 Nuclear Chemistry Counting Facility Manager 7/03 - 7/04 Sabbatical in the Chemistry Dept., University of Maryland w/Dr. William B. Walters 
7/03 - Present Visiting Professor, Chemistry Dept., Univ. of Maryland

7/03 - Present Senior Staff Scientist (26 years LLNL service in Jan. 2011)

06/95 to Present Guest LBNL, Berkeley, CA

2/07 - present Experimental Nuclear Physics Group Leader, Physics Div., LLNL

\section{Professional Memberships:}

American Physical Society (Nuclear Physics Division)

American Chemical Society (Nuclear Chemistry Division)

American Association for the Advancement of Science

Sigma Chi Science Society

\section{Awards and Honors:}

NNSA Certificate of Appreciation, 2009.

13 Directorate Awards, 1999-2011.

US DOE certificate of appreciation, 2008.

Distinguished service award, 2004.

\section{Professional Leadership and Service:}

- American Chemical Society, Past Chair, from 2010 to 2011.

- American Chemical Society, Chair, from 2009 to 2010.

- American Chemical Society, Chair Elect, from 2008 to 2009.

- American Chemical Society, Vice Chair, from 2007 to 2008.

- Los Alamos National Laboratory, LANSCE Users Group Executive Committee, Member from 2008 to 2010.

- $\quad$ NNSA, Stockpile Stewardship Academic Alliance, Proposal Reviewer from 2005 to present.

- DOE/SC/NP, Nuclear Physics, Proposal Reviewer from 2004 to present .

- Los Alamos National Laboratory, LANSCE Program Advisory Committee, Member from 2001 to 2003.

- $\quad$ Referee Phys. Rev. C, Phys. Rev. Lett., NIMA, Radiochem. Acta, J. Phys. G, Nature

\section{Three most important publications:}


1. “Intense High Energy Proton Beams from Petawatt Laser Irradiation of Solids” R.A. Snavely, M.H. Key, S.P. Hatchett, T.E. Cowan, M. Roth, T.W. Phillips, M.A. Stoyer, E.A. Henry, T.C. Sangster, M.S. Singh, S.C. Wilks, A. MacKinnon, A. Offenberger, D.M Pennington, K. Yasuike, A.B. Langdon, B.F. Lasinski, J. Johnson, M.D. Perry, and E.M Campbell, Phys. Rev Lett. 85 (2000) 2945.

2. "Synthesis of Superheavy Nuclei in the ${ }^{48} \mathrm{Ca}+{ }^{244} \mathrm{Pu}$ Reaction" Yu.Ts.Oganessian, V.K.Utyonkov, Yu.V.Lobanov, F.Sh.Abdullin, A.N.Polyakov, I.V.Shirokovsky, Yu.S.Tsyganov, G.G.Gulbekian, S.L.Bogomolov, B.N.Gikal, A.N.Mezentsev, S.Iliev, V.G.Subbotin, A.M.Sukhov, G.V.Buklanov, K.Subotic, M.G.Itkis, K.J.Moody, J.F.Wild, N.J.Stoyer, M.A.Stoyer, R.W.Lougheed, Phys. Rev. Lett. 83 (1999) 3154.

3. "Synthesis of a New Element with Atomic Number $Z=117$ ” Yu. Ts. Oganessian, F.S. Abdullin, P.D. Bailey, et al., Phys. Rev. Lett. 104 (2010) 142502. 
LLNL-TR-497871

\begin{tabular}{|c|c|c|c|c|c|c|c|c|}
\hline & $\begin{array}{r}\text { Budg } \\
\text { w Energy } \\
\text { At Na }\end{array}$ & $\begin{array}{l}\text { rm For } \\
\text { arch Prog } \\
\text { Laborato }\end{array}$ & $\begin{array}{l}\text { grams } \\
\text { ories }\end{array}$ & & & & & \\
\hline $\begin{array}{l}\text { ORGANIZATION } \\
\text { FWPA } \quad \text { SCW0147 }\end{array}$ & & & & & & & & \\
\hline 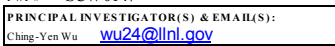 & & & & & & & & \\
\hline $\begin{array}{l}\begin{array}{l}\text { Budget Page \# ( ) } \\
\text { VERSION () DATE: ( Aug. 1,2011) }\end{array} \\
\end{array}$ & & & \begin{tabular}{|l} 
LE \\
All costs a
\end{tabular} & $\frac{\text { RESEARC }}{\text { ire fully }}$ & $\begin{array}{ll}\text { HFUNDS } \\
\text { Hurdened }\end{array}$ & $(\mathrm{KB} 0401)$ & & \\
\hline $\begin{array}{l}\text { A. SCIENTIFIC PERSONNELSTAFF } \\
\text { One line for each person }\end{array}$ & NP Funde & in $F Y 11$ & \begin{tabular}{|c|} 
Actual \\
FY 2011
\end{tabular} & & & & & \\
\hline List additional personncl on a 2 nd shect; show SUBTOTALon linc 7 & & Ressarch & (Res.only) & FY 2012 & FY 2013 & FY 2014 & FY 2015 & FY 2016 \\
\hline Ching-Yen Wu & & \begin{tabular}{|c|c|}
0.10 & 0 \\
\end{tabular} & 40.0 & 40.0 & 40.6 & 41.2 & 41.8 & \\
\hline 2. & & & & & & & & \\
\hline 3. & & & & & & & & \\
\hline 4. & & & & & & & & \\
\hline 5. & & & & & & & & \\
\hline 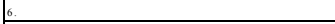 & & & & & & & & \\
\hline \begin{tabular}{|ll}
7 & SUB TOTALOF ADDITONALPER SONNELLshow breakdown
\end{tabular} & na 2 nd page) & & & & & & & \\
\hline $\begin{array}{|lc|}8 . & (\quad) \text { TOTALSCIENTFIF PERSONNEL (1-7) } \\
\end{array}$ & & 0.10 & 40.0 & 40.0 & 40.6 & 41.2 & 41.8 & 0.0 \\
\hline B. OTHER Scientific Perso nnel (Show number of peo & It in bracke & vide names & $s$ details on & explanatio & in sheet) & & & \\
\hline $\left.\begin{array}{|ll}1 .( & 0.6\end{array}\right)$ Term Appointments, including POST DOCS & & 0.75 & 125.0 & 145.0 & 147.2 & 149.4 & 151.6 & \\
\hline \begin{tabular}{|l|l|}
$2 .(1)$ GRADUATE STUDENTS (Stipend) \\
\end{tabular} & & 0.00 & & & & & & \\
\hline \begin{tabular}{|l|l|}
$3 .($ & JVIITNG SCENTISTS \\
\end{tabular} & & 0.00 & & & & & & \\
\hline $4.4 \quad$ OTHER PROFESSIONAL(TECH, $\Pi$, PROGRAMMER & & 0.00 & & & & & & \\
\hline 5.1 , SECRETARIAL-CLERICAL. & & 0.00 & & & & & & \\
\hline \begin{tabular}{|l|l|}
$6 .($ & ) ANY OTHER (e.g, Visitors) \\
\end{tabular} & & 0.00 & & & & & & \\
\hline \begin{tabular}{|l|} 
SUB TOTALSALARIES AND WAGES (B) \\
\end{tabular} & & & 125.0 & 145.0 & 147.2 & 149.4 & 151.6 & 0.0 \\
\hline C. TOTAL Fully Burdened SALARIES $(A+B)$ & & & 165.0 & 185.0 & 187.8 & 190.6 & 193.5 & 0.0 \\
\hline D. PERMANENT EQUIPMENT (Not applicable to La & & & & & & & & \\
\hline TOTALPERMANENT EQUIPMENT & & & 0.0 & 0.0 & 0.0 & 0.0 & 0.0 & 0.0 \\
\hline \begin{tabular}{|ll} 
E. & TRAVEL (STAFF \& POSTDOCS) 1 DOMESTTC \\
\end{tabular} & & & & & & & & \\
\hline 2. FOREGIN & & & & & & & & \\
\hline Provide breakdown n explanation shect; $;$.g., experiment, me. & ngs \& conferer & & & & & & & \\
\hline TOTAL TRAVEL & & & 10.0 & 10.0 & 10.2 & 10.3 & 10.5 & 0.0 \\
\hline F. Fully burdened STUDENT/TRAINEE $\cos$ TS (Shon & names, \&u & iliations on & explanation & isheet) & & & & \\
\hline 1. TUIIION \& FEES GRADUTE STUDENTS & & & & & & & & \\
\hline 2. TUITION \&FEES UNDR GRADUATE STUDENTS & & & & & & & & \\
\hline 3. TRAVEL(Provide breakd own on explanation shect; c.eg. & $\mathrm{xperiment,} \mathrm{mc}$ & conferences,, $\mathrm{c}$ & & & & & & \\
\hline 4. отHЕR & & & & & & & & \\
\hline \begin{tabular}{|l} 
TOTALSTUDENT/TRANEE COSTS \\
\end{tabular} & & & 0.0 & 0.0 & 0.0 & 0.0 & 0.0 & 0.0 \\
\hline G. OTHER $\cos \mathrm{TS}$ & & & & & & & & \\
\hline $\begin{array}{l}\text { 1. MATERIALS AND SUPPLES } \\
\end{array}$ & & & & & & & & \\
\hline 2. PUBLCATON COSTS/DOCUMENTATION/DISSEMIN & & & & & & & & \\
\hline 3. CONSULTANT SERVICES & & & & & & & & \\
\hline 4. Ir \&COMPUTER SERVICES not covered under indirect & & & & & & & & \\
\hline 5. SUBCONTRACTS & & & & & & & & \\
\hline 6. OTHER & & & & & & & & \\
\hline TOTALOTHER COSTS & & & 0.0 & 0.0 & 0.0 & 0.0 & 0.0 & 0.0 \\
\hline H. TOTAL $\cos T S$ For A THROUGH G & & & 175.0 & 195.0 & 197.9 & 200.9 & 203.9 & 0.0 \\
\hline Scnario A & & & & & & & & \\
\hline 1. Cost of Living Adjustment assumption & & & $0 \%$ & $0 \%$ & $1.5 \%$ & $1.5 \%$ & $1.5 \%$ & \\
\hline I. CARRY FORWARD \& RESERVES (acc rued from & ior years) & & & & & & & \\
\hline 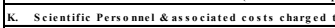 & PROJECTS & & & & & & & \\
\hline \begin{tabular}{|l} 
L. Scientific Personnel \& as sociated costs charged \\
\end{tabular} & WFo & & & & & & & \\
\hline 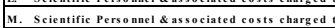 & LDRD & & & & & & & \\
\hline $\begin{array}{l}\text { N. Scientific Perso nnel \& associated costs charged } \\
\end{array}$ & Org B urde & & & & & & & \\
\hline O. Scientific Personel \& associated costs charged & Fellows hip & vards of fin & nite duration & & & & & \\
\hline $\begin{array}{l}\text { COMMENTS \& NOTES (identify each item } \\
\text { B1. Elaine Kwan }\end{array}$ & by a labe & ., F.3); & 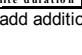 & nal $\mathrm{r}$ & as 1 & & & \\
\hline
\end{tabular}

\begin{tabular}{|c|c|c|c|c|c|}
\hline FY 2010 & FY 2011 & FY 2012 & $\mathrm{FY} 2013$ & FY 2014 & $\mathrm{FY} 2015$ \\
\hline 0.05 & 0.1 & 0.1 & 0.09 & 0.09 & 0.08 \\
\hline & & & & & \\
\hline & & & & & \\
\hline & & & & & \\
\hline & & & & & \\
\hline & & & & & \\
\hline B. OTHER Scien & ic Personnel & & & & \\
\hline 0.6 & 0.6 & 0.6 & 0.6 & 0.6 & 0.6 \\
\hline & & & & & \\
\hline & & & & & \\
\hline & & & & & \\
\hline & & & & & \\
\hline
\end{tabular}
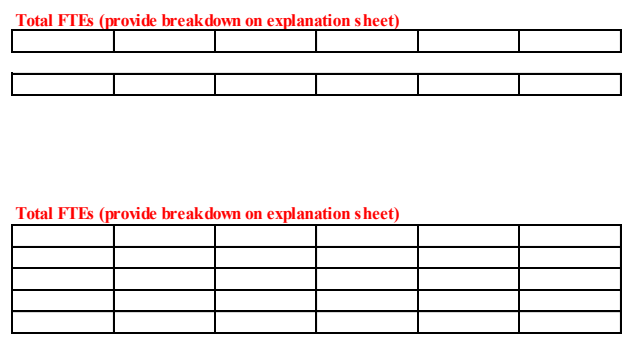
LLNL-TR-497871

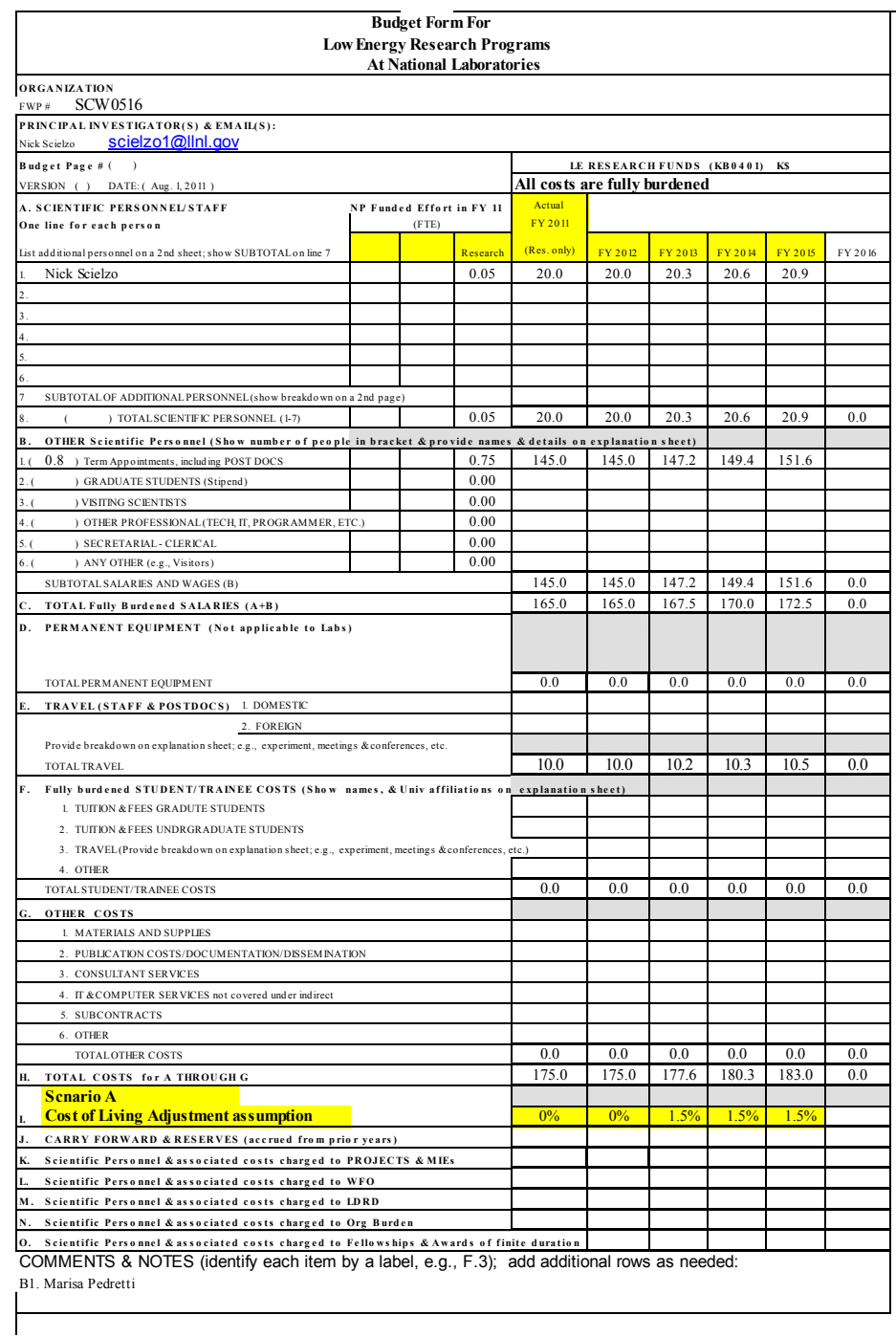

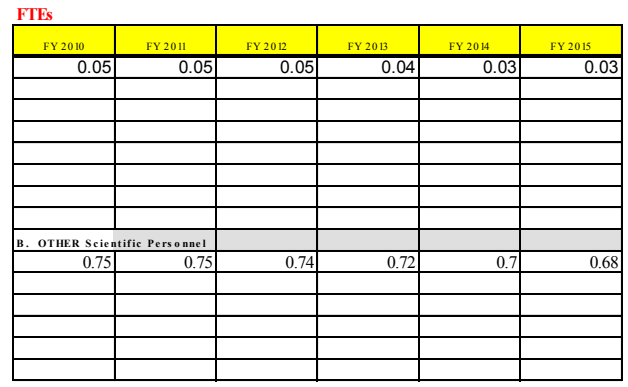
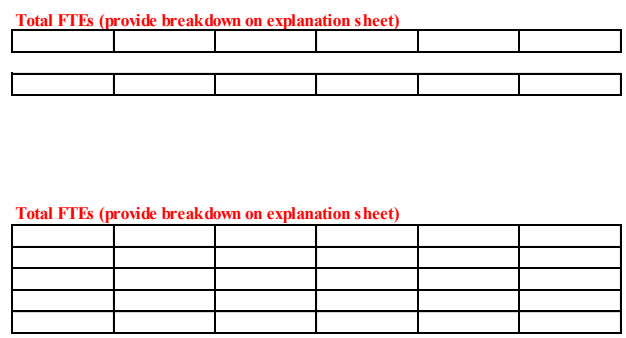
LLNL-TR-497871

\begin{tabular}{|c|c|c|c|c|c|c|c|c|}
\hline & $\begin{array}{r}\text { Budg } \\
\text { w Energy } \\
\text { At Na }\end{array}$ & $\begin{array}{l}\text { rm For } \\
\text { arch Prog } \\
\text { Laborato }\end{array}$ & $\begin{array}{l}\text { grams } \\
\text { ories }\end{array}$ & & & & & \\
\hline $\begin{array}{l}\text { ORGANIZATION } \\
\text { FWPA } \quad \text { SCW0147 }\end{array}$ & & & & & & & & \\
\hline 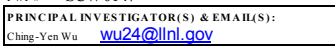 & & & & & & & & \\
\hline $\begin{array}{l}\begin{array}{l}\text { Budget Page \# ( ) } \\
\text { VERSION () DATE: ( Aug. 1,2011) }\end{array} \\
\end{array}$ & & & \begin{tabular}{|l} 
LE \\
All costs a
\end{tabular} & $\frac{\text { RESEARC }}{\text { ire fully }}$ & $\begin{array}{ll}\text { HFUNDS } \\
\text { Hurdened }\end{array}$ & $(\mathrm{KB} 0401)$ & & \\
\hline $\begin{array}{l}\text { A. SCIENTIFIC PERSONNELSTAFF } \\
\text { One line for each person }\end{array}$ & NP Funde & in $F Y 11$ & \begin{tabular}{|c|} 
Actual \\
FY 2011
\end{tabular} & & & & & \\
\hline List additional personncl on a 2 nd shect; show SUBTOTALon linc 7 & & Ressarch & (Res.only) & FY 2012 & FY 2013 & FY 2014 & FY 2015 & FY 2016 \\
\hline Ching-Yen Wu & & \begin{tabular}{|c|c|}
0.10 & 0 \\
\end{tabular} & 40.0 & 41.2 & 42.4 & 43.7 & 45.0 & \\
\hline 2. & & & & & & & & \\
\hline 3. & & & & & & & & \\
\hline 4. & & & & & & & & \\
\hline 5. & & & & & & & & \\
\hline ( & & & & & & & & \\
\hline 7 SUB TOTALOF ADDITIONALPERSONNELL(show breakdown & na 2nd page) & & & & & & & \\
\hline $\begin{array}{|lc|}8 . & (\quad) \text { TOTALSCIENTFIF PERSONNEL (1-7) } \\
\end{array}$ & & 0.10 & 40.0 & 41.2 & 42.4 & 43.7 & 45.0 & 0.0 \\
\hline B. OTHER Scientific Perso nnel (Show number of peo & It in bracke & vide names & $s$ details on & explanatio & in sheet) & & & \\
\hline $\left.\begin{array}{|ll}1 .( & 0.6\end{array}\right)$ Term Appointments, including POST DOCS & & 0.75 & 125.0 & 128.8 & 132.6 & 136.6 & 140.7 & \\
\hline \begin{tabular}{|l|l|}
$2 .(1)$ GRADUATE STUDENTS (Stipend) \\
\end{tabular} & & 0.00 & & & & & & \\
\hline \begin{tabular}{|l|l|}
$3 .($ & , VISTING SCENTISTS \\
\end{tabular} & & 0.00 & & & & & & \\
\hline $4.4 \quad$ OTHER PROFESSIONAL(TECH, $\Pi$, PROGRAMMER & & 0.00 & & & & & & \\
\hline 5.1 , SECRETARIAL-CLERICAL. & & 0.00 & & & & & & \\
\hline \begin{tabular}{|l|l|}
$6 .($ & ) ANY OTHER (e.g, Visitors) \\
\end{tabular} & & 0.00 & & & & & & \\
\hline \begin{tabular}{|l|} 
SUB TOTALSALARIES AND WAGES (B) \\
\end{tabular} & & & 125.0 & 128.8 & 132.6 & 136.6 & 140.7 & 0.0 \\
\hline C. TOTAL Fully Burdened SALARIES $(A+B)$ & & & 165.0 & 170.0 & 175.0 & 180.3 & 185.7 & 0.0 \\
\hline D. PERMANENT EQUIPMENT (Not applicable to La & & & & & & & & \\
\hline TOTALPERMANENT EQUIPMENT & & & 0.0 & 0.0 & 0.0 & 0.0 & 0.0 & 0.0 \\
\hline \begin{tabular}{|ll} 
E. & TRAVEL (STAFF \& POSTDOCS) 1 DOMESTTC \\
\end{tabular} & & & & & & & & \\
\hline 2. FOREGIN & & & & & & & & \\
\hline Provide breakdown n explanation shect; $;$.g., experiment, me. & ngs \& conferer & & & & & & & \\
\hline TOTAL TRAVEL & & & 10.0 & 10.3 & 10.6 & 10.9 & 11.3 & 0.0 \\
\hline F. Fully burdened STUDENT/TRAINEE $\cos$ TS (Shon & names, \&u & iliations on & explanation & isheet) & & & & \\
\hline 1. TUIIION \& FEES GRADUTE STUDENTS & & & & & & & & \\
\hline 2. TUTrON \&FEES UNDR GRADUATE STUDENTS & & & & & & & & \\
\hline 3. TRAVEL(Provide breakd own on explanation shect; c.e.g. & $\mathrm{xperiment,} \mathrm{mc}$ & conferences,, $\mathrm{c}$ & & & & & & \\
\hline 4. отHЕR & & & & & & & & \\
\hline \begin{tabular}{|l} 
TOTALSTUDENT/TRANEE COSTS \\
\end{tabular} & & & 0.0 & 0.0 & 0.0 & 0.0 & 0.0 & 0.0 \\
\hline G. OTHER $\cos \mathrm{TS}$ & & & & & & & & \\
\hline $\begin{array}{l}\text { 1. MATERIALS AND SUPPLES } \\
\end{array}$ & & & & & & & & \\
\hline 2. PUBLCATON COSTS/DOCUMENTATION/DISSEMIN & & & & & & & & \\
\hline 3. CONSULTANT SERVICES & & & & & & & & \\
\hline 4. Ir \&COMPUTER SERVICES not covered under indirect & & & & & & & & \\
\hline 5. SUBCONTRACTS & & & & & & & & \\
\hline 6. OTHER & & & & & & & & \\
\hline TOTALOTHER COSTS & & & 0.0 & 0.0 & 0.0 & 0.0 & 0.0 & 0.0 \\
\hline H. TOTAL $\cos T S$ For A THROUGH G & & & 175.0 & 180.3 & 185.7 & 191.2 & 197.0 & 0.0 \\
\hline Scnario B & & & & & & & & \\
\hline 1. Cost of Living Adjustment assumption & & & $0 \%$ & $3 \%$ & $3 \%$ & $3 \%$ & $3 \%$ & \\
\hline I. CARRY FORWARD \& RESERVES (acc rued from & ior years) & & & & & & & \\
\hline K. Scientific Personnel \& associated costs charged & PROJECTS & & & & & & & \\
\hline L. Scientific Personnel \& as sociated costs charged & WFo & & & & & & & \\
\hline 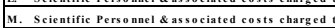 & LDRD & & & & & & & \\
\hline $\begin{array}{l}\text { N. Scientific Perso nnel \& associated costs charged } \\
\end{array}$ & Org B urde & & & & & & & \\
\hline O. Scientific Personel \& associated costs charged & Fellows hip & vards of fin & nite duration & & & & & \\
\hline $\begin{array}{l}\text { COMMENTS \& NOTES (identify each item } \\
\text { B1. Elaine Kwan }\end{array}$ & by a labe & ., F.3); & 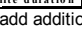 & aal $\mathrm{r}$ & as 1 & & & \\
\hline
\end{tabular}

\begin{tabular}{|c|c|c|c|c|c|}
\hline Fr & 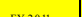 & & -5 & - & - \\
\hline 0.05 & 0.1 & 0.1 & 0.1 & 0.1 & 0.1 \\
\hline & & & & & \\
\hline & & & & & \\
\hline & & & & & \\
\hline & & & & & \\
\hline & & & & & \\
\hline B. OTHER Scien & ic Personnel & & & & \\
\hline 0.6 & 0.6 & 0.6 & 0.6 & 0.6 & 0.6 \\
\hline & & & & & \\
\hline & & & & & \\
\hline & & & & & \\
\hline & & & & & \\
\hline
\end{tabular}
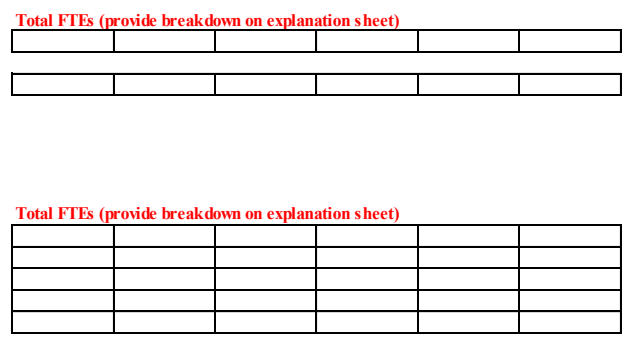
LLNL-TR-497871

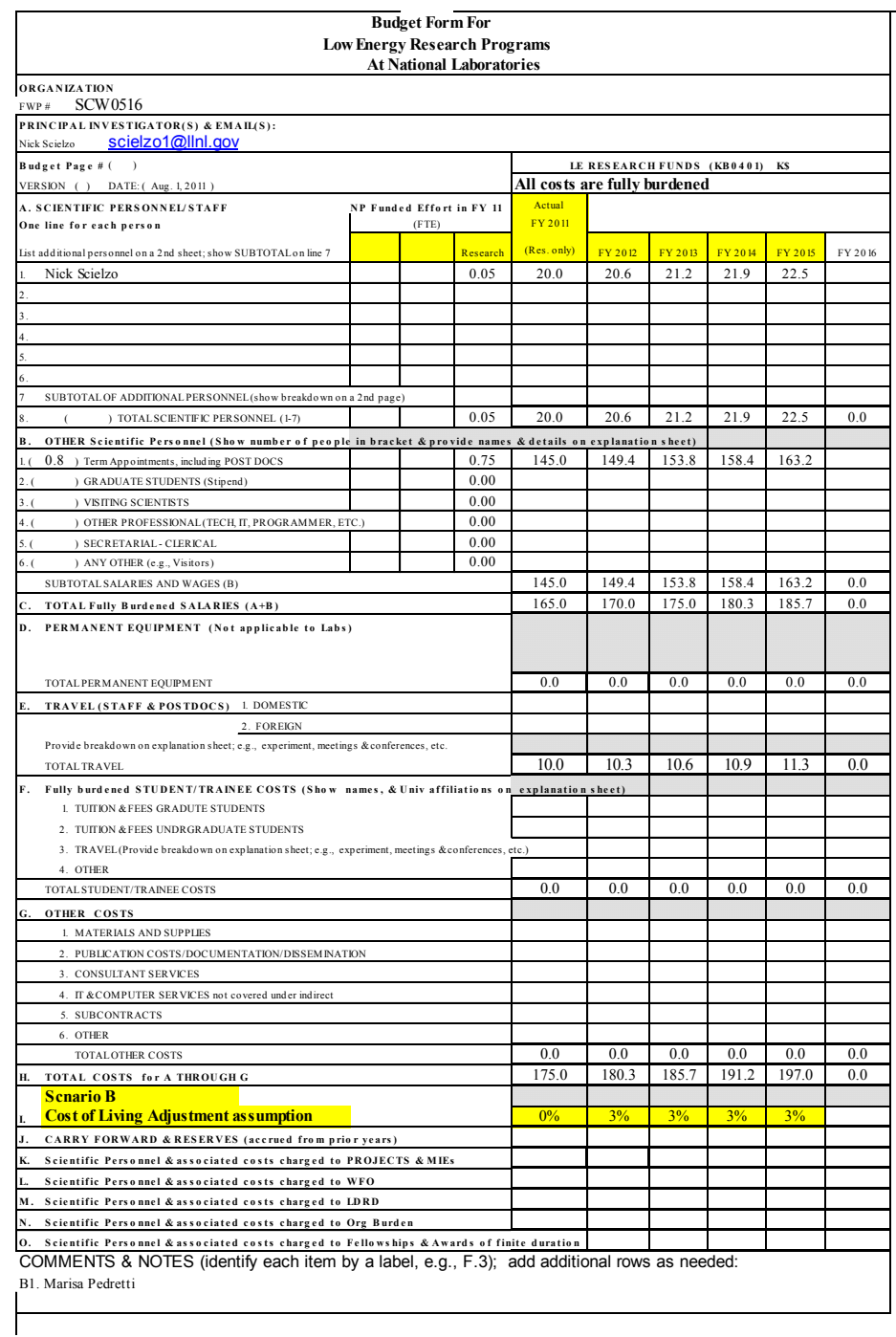

FTEs
\begin{tabular}{|r|r|r|r|r|r|}
\hline FY 2010 & FY 2011 & FY 2012 & FY 2013 & FY 2014 & FY 2015 \\
\hline \hline 0.05 & 0.05 & 0.05 & 0.05 & 0.04 & 0.04 \\
\hline & & & & & \\
\hline & & & & & \\
\hline & & & & & \\
\hline & & & & & \\
\hline & & & & & \\
\hline & & & & & \\
\hline & & & & & \\
\hline B. OTHER Scientific Personne1 & & & & \\
\hline 0.75 & 0.75 & 0.75 & 0.75 & 0.75 & 0.75 \\
\hline & & & & & \\
\hline & & & & & \\
\hline & & & & & \\
\hline & & & & & \\
\hline & & & & &
\end{tabular}
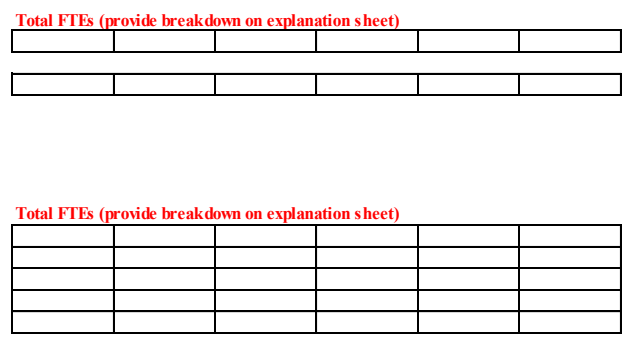
LLNL-TR-497871

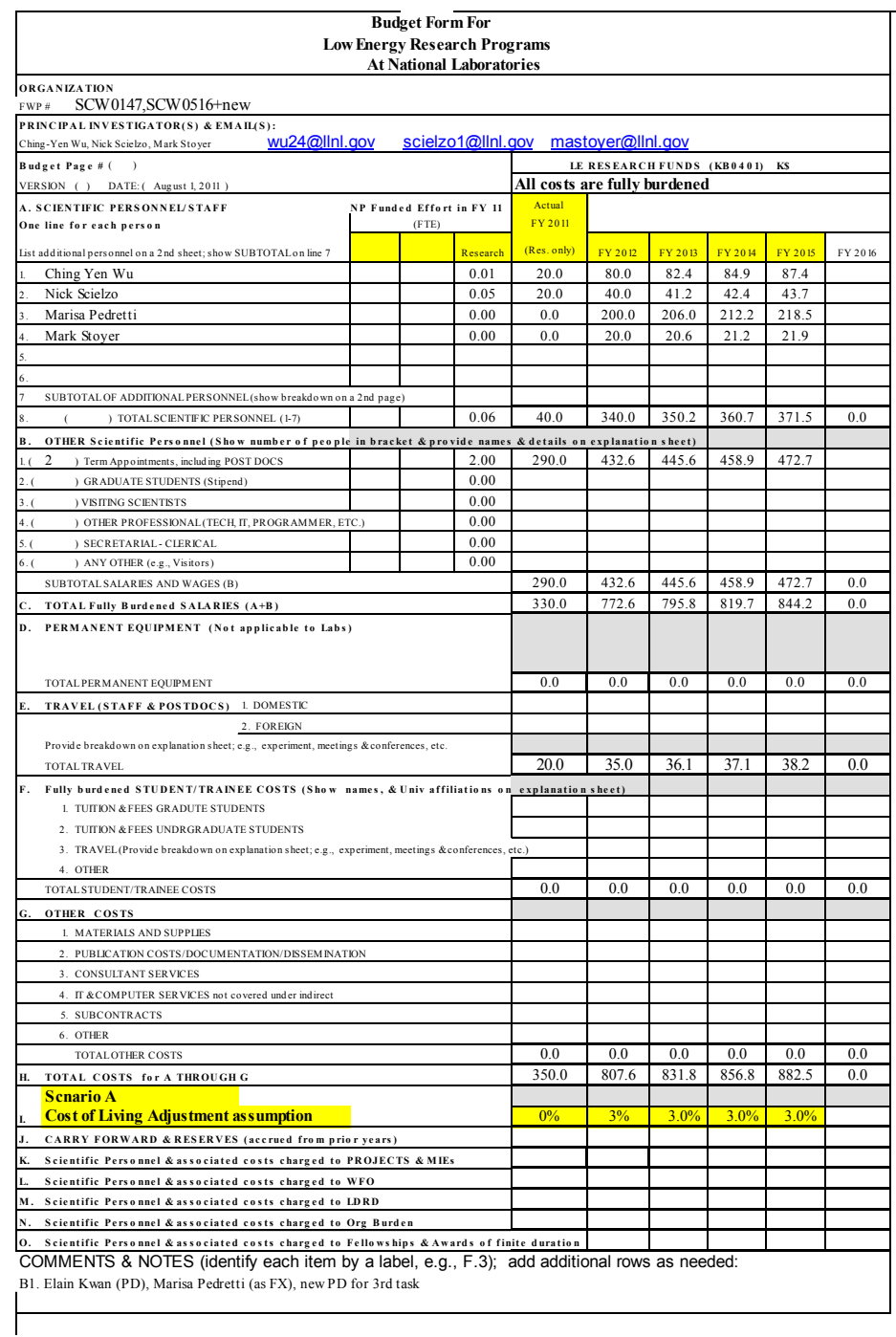
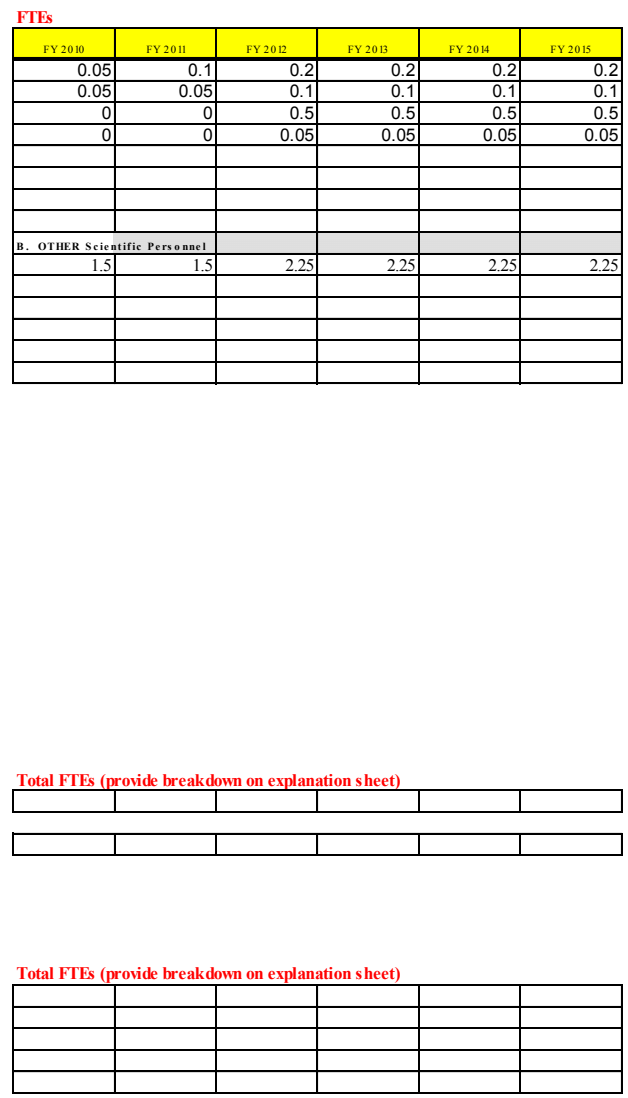


\section{Budget Justification/Explanation}

Budget scenario A and B spreadsheets were prepared using required guidance and therefore don't require additional explanation.

The grand-total proposed budget for these efforts (+ new SHE effort) requires further explanation. It should be noted that LLNL is a laboratory different than Office of Science laboratories in that we leverage funding from other parts of DOE such as NNSA, DNDO, and NA22 and even other funding agencies (DOD).

\section{Exotic Nuclei Studies}

The FY11 budget for this effort was $\$ 175 \mathrm{~K}$. Not included in the budget sheets was the one time additional $\$ 370 \mathrm{~K}$ for Chico2 development. The FY11 funding supported Ching-Yen Wu at 10\% time and a PD (Elaine Kwan) at $60 \%$ time (LLNL G\&A supports $25 \%$ of a PD for career development activities). The remaining funding was used for travel to experiments (approximately one trip per year to TRIUMF, ANL and MSU) and some materials. We request a small amount of additional funding FY12-FY15 ( $\$ 40 \mathrm{~K})$ to increase Ching-Yen's time to $20 \%$ effort. This additional funding is required for development of experiments at MSU moving towards FRIB and support of experiments at TRIUMF, ANL and MSU.

\section{CUORE Neutrinoless Double-beta Decay}

The FY11 budget for this effort was $\$ 175 \mathrm{~K}+\$ 250 \mathrm{~K}$ additional allocated in Aug. 2011 as an advance which was not added to base funding. We continue to request an additional amount of funding to be added to the base starting in FY13 in order to achieve $\sim 425 \mathrm{~K}$ level principally for support Marisa as a staff person rather than PD. The total amount is included in the budget sheets distributed as funding to convert Marisa Pedretti (currently PD) to staff and support her at 50\% (other 50\% support is programmatic), to increase Nicholas Scielzo to $10 \%$ effort, and to increase travel funding by $\sim \$ 20 \mathrm{~K}$. The LLNL group currently consists of Nicholas Scielzo (staff) and Marisa Pedretti (post-doc), who are funded through the Office of Science and Eric Norman (staff), and Barbara Wang (grad student), who are funded through UC Berkeley and external funding, and Kareem Kazkaz (staff), and Samuele Sangiorgio (post-doc), who participate in the LLNL CUORE effort but currently receive no funding through Office of Science (they receive funding through LLNL or other sources). The group is looking to add a graduate student through the Nuclear Science and Security Consortium led by UC Berkeley Nuclear Engineering Department. Marisa Pedretti is currently a post-doctoral researcher at LLNL who started in January 2009 and it is LLNL's intention to hire her as a research staff member before her postdoc appointment ends in January 2012. In addition, LLNL contributes an additional 0.25 FTE 
per year for each post-doc from a G\&A account for career development activities. A new postdoc will be supported at the 0.75 FTE level after Marisa Pedretti's term as a post-doc ends in FY12. In FY08-10, the group has leveraged LLNL resources of a Lawrence Postdoctoral Fellowship (Nicholas Scielzo) and a three-year Exploratory Research LDRD.

The LLNL group currently has much of the US CUORE team members that developed the US Monte Carlo simulation to study scientific and background signatures. With the additional funding, the LLNL group effort will be able to implement the four-year research plan described above including participating in the background determination and elimination in the bolometers and overseeing two graduate student theses on these backgrounds and a search for exotic double beta decays.

Travel:

Domestic:

Yearly travel to 1 domestic CUORE collaboration meeting for three LLNL members. Yearly travel to 1 domestic conference for two LLNL members.

International:

In FY11 only, Marisa Pedretti will travel to LNGS for 9-12 months for her role as the CUORE-0 experimental coordinator.

Yearly travel to 2 CUORE collaboration meetings for three LLNL members.

For FY12-15, travel (14 weeks total) is anticipated for experimental shifts at LNGS for the LLNL group.

Yearly travel to 1 foreign conference for two LLNL members.

\section{SHE Automated Chemistry Development}

New funding of $\$ 150 \mathrm{~K}$ is requested for support of 0.5-0.75 PD (institution supports 0.25 ), travel, and some material expenses - to investigate the chemistry of the heaviest elements using an automated chemical apparatus. We have a candidate PD, but this person would still have to be hired. This request is the source of $\$ 150 \mathrm{~K}$ increase in FY12 budget and additional FY13-15 outyears. Without additional funding, this effort would not be performed.

\section{Summary}

The large increase from FY11 to FY12 $(\$ 350 \mathrm{~K} \rightarrow \$ 807 \mathrm{~K})$, a $\$ 457 \mathrm{~K}$ increase, is explained as follows: the $\$ 250 \mathrm{~K}$ Aug. 2011 CUORE-0 advance is included here, the new $\$ 150 \mathrm{~K}$ SHE request is included here, and an additional $\$ 50 \mathrm{~K}$ for exotic nuclei in order to increase Ching-Yen's time to $20 \%$. 


\section{Description of LLNL BURDENS}

\section{PERSONNEL COSTS:}

Payroll Burden costs are incurred for employee fringe benefits and employer paid payroll taxes and employee insurance. $56 \%$

\section{OTHER DISTRIBUTED CHARGES:}

> Program Management Charge (PMC) costs are incurred for managing and administering programs within a Principal Directorate. Major activities/functions include Program Management, Financial Management, Program Development, and other indirect functions in support of direct programmatic activities. Varies

> General \& Administrative (G\&A) costs cover management, financial, or other expenses incurred for the general management and administration of the Laboratory as a whole. Activities include Senior Management Administration, External Relations \& Communications, Staff Relations, Legal, Internal Audit, Human Resources, Finance and Budget, Procurement, Prime Contract Management, Integrated Performance and Pay Administration, and non-programmatic workforce management. $17.6 \%$

There is a special allocation distributed to the National Ignition Campaign (NIC), via the G\&A NIC rate, for G\&A functions/activities specifically identifiable with NIC construction. Some Standard G\&A functions are the same as the G\&A NIC, but only costs that are caused by or specifically identifiable with NIC construction are included in the G\&A NIC.

> Hazardous Waste Charge (HWC) is allocated to all Non-DOE Workfor-Other (WFO) projects and represents the Non-DOE WFO programs' share of LLNL's Waste Management costs. $1.5 \%$

> Lab Directed Research \& Development (LDRD) is an assessment annually approved by the DOE that funds research and development work selected by the Laboratory's Director or designee that enhances the scientific and technological vitality of the Lab. $9.1 \%$

> DOE Federal Administrative Charge (FAC) represents an allocation of DOE's depreciation and administrative costs to Non-DOE funded projects. $3 \%$

> Safeguards and Security Charge (S\&S) is allocated to all Non-DOE Work-for-Other (WFO) projects and represents the Non-DOE WFO programs' share of LLNL's Safeguards and Security costs. $2.3 \%$ 
$>$ Strategic Mission Support (SMS) costs are incurred for science and technology strategic planning, outreach, special employees, library, institutional capabilities, and other activities that enhance the Laboratory's ability to address future missions.

Management Fee is paid to LLNS, LLC for oversight and management of the Laboratory. Maximum available fee is established in the contract and consists of a fixed and performance incentive portion. The fee is applied to DOE, DHS, NNSA, and work for others funds, except for those cost estimates where fee is specifically excluded by contract. There are 3 management fee rates in effect: DOE, non-DOE (reimbursable work including DHS), and National Ignition Campaign (NIC).

- Site Support covers the costs necessary for the management, maintenance, and upgrades of the general purpose facilities and property, and for the provision of basic infrastructure services and safety on-site. Activities include Computer Support, Networks, Telecommunications, Information Technology Management \& Services, Health Services, Environmental Protection, Hazards Control, Site Emergency Preparedness, Facilities Management, Facilities Maintenance, Roads \& Grounds Maintenance, Fire Protection Services, ES\&H Compliance, Shipping, Receiving, Mail Services, and Taxi Services. Costs in the pool also include Institutional General Purpose Equipment (IGPE) and Institutional General Plant Projects (IGPP), which consist of capital equipment and construction projects of a general use or institutional nature that is required for general purpose site wide needs. The Site Support NIC is a special allocation distributed to the National Ignition Campaign (NIC) project via a Site Support NIC rate. 


\section{NNS:}

\author{
U. S. Department of Energy \\ National Nuclear Security Administration \\ Livermore Site Office \\ PO Box 808, L-293 \\ 7000 East Avenue \\ Livermore, California 94551-0808
}

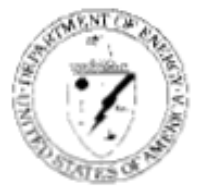

\section{OCT 012010}

\author{
Ms. Linda Rakow \\ Chief Financial Officer \\ Lawrence Livermore National Security, LLC \\ Lawrence Livermore National Laboratory \\ 7000 East Avenue, L-435 \\ Livermore, CA 94550 \\ Subject: Contract DE-AC52-07NA27344, Clause I-110, Approval of Revised Provisional \\ Billing and Forward Pricing Rates for Fiscal Year 2011
}

Dear Ms. Rakow:

The enclosed Fiscal Year (FY) 2011 rates proposed by Lawrence Livermore National Security, LLC (LLNS) on September 30, 2010, are hereby approved for forward pricing and for interim billing during FY 2011.

In accordance with Federal Acquisition Regulation 42.704, rates may be prospectively or retroactively revised by mutual agreement, or unilaterally determined by the Contracting Officer, pending establishment of final indirect cost rates. Additionaliy, the elements of indirect cost and the base or bases used in computing billing rates shall not be construed as determinative of the indirect costs to be distributed or of the bases of distribution to be used in the final settlement.

LLNS shall continue to submit a comparison of actual rates versus approved forward pricing and billing rates for all indirect cost pools and standard salary rates on a quarterly basis for the first six months of the FY and on a monthly basis thereafter. In accordance with the approved Performance Evaluation Plan, estimated year-end variances between actual and approved rates shall be managed to within the lower of $5 \%$ of pool costs or $\$ 10$ million by pool.

If you should have any questions, please contact me at (925) 424-2986.

Sincercly,

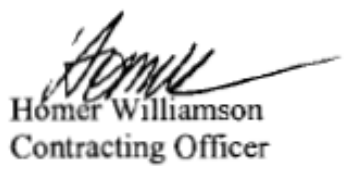


Enclosure: 10-009DP (D. Payne/H. Williamson), Proposed FY2011 Billing Rates, dated September 30,2010
cc
G. Gilliland, OFFM
J. Parenti
D. Goett
D. Boyd
A. Moser
D. Payne
K. Baker
P. Rosenkoetter 


\section{Current or pending support:}

N.D. Scielzo received LDRD funding (\$150-200k per year) as PI for "Research and Development for a Cryogenic Bolometer for Double-Beta Decay Research" (08-ERD-049) over the three-year period FY08-10. This successful project developed new techniques to improve the sensitivity of bolometric detectors to rare radioactive decays such as the long-sought-after neutrinoless double-beta decay by reducing detector backgrounds, improving detector instrumentation methods, and developing analysis tools to determine the source of any remaining backgrounds and the sensitivity to various scientific signals of interest. This research has had an immediate impact on the CUORE project as the tools developed to instrument bolometers with thermistor and heater chips is being used in the CUORE-0 assembly and will ultimately be used to instrument all the CUORE crystals. The LLNL group will continue to seek funding through LLNL LDRD to support research and development efforts related to advanced scintillating and/or isotopically enriched bolometers. 


\section{Student education and training supported by the group:}

\section{Exotic Nuclear Studies}

Education is an important component of this program. Many students including the undergraduates and postdocs are involved in our fielded experiments. A total of five Ph.D.'s were awarded over a period of 16 years in our Gammasphere/CHICO collaboration. One Ph.D. was awarded so far and more in the pipeline for our TIGRESS/Bambino collaboration. We anticipate numerous Ph.D.'s will be awarded in the future for our newly formed JANUS collaboration with MSU. These collaborations pursue the physics of mutual interest but also provide a fertile ground to nurture the next generation of nuclear physicists.

\section{CUORE neutrinoless Double-beta Decay Studies}

The LLNL CUORE group has had a strong connection with UC Berkeley through Eric Norman of the Department of Nuclear Engineering:

Michelle Dolinski was funded through a LLNL fellowship (the SEGRF fellowship, now called "Lawrence Scholarship") to work with the LLNL group. Her thesis topic was "Neutron Interactions in the CUORE Neutrinoless Double Beta Decay Experiment". She graduated in the fall of 2008 and has since taken a post-doctoral researcher position in neutrino physics at Stanford Univeristy.

Barbara Wang has joined the LLNL group and is funded through a Nuclear Forensics Graduate Fellowship through the Department of Homeland Security. She will be analyzing the backgrounds that are expected in the CUORE experiment and in particular the signals at both low energies and in the $0 v \beta \beta$ region that result from cosmogenic activation.

The LLNL group is expecting to attract a second graduate student from the "Nuclear Science and Security Consortium" that was recently awarded by NNSA to a UC Berkeley led team that included LLNL. The LLNL group's CUORE research was featured as one of the research topics for graduate students. This Consortium would provide funding for a graduate student to work at Livermore with the LLNL group.

In addition, a UC Berkeley undergraduate student, Jessica Mintz, was involved in the tellurium double-beta decay $Q$ value measurements during 2008-2009. She played an important role in collecting the data using the Penning trap and travelled to Gran Sasso to learn about the CUORE experiment and participate in the onsite research and development activities. She is currently attending graduate school in nuclear engineering at Purdue University. 


\title{
PAC34 - LETTER OF INTENT
}

\author{
Low-energy Coulomb excitation, inelastic scattering and transfer reaction \\ measurements at ReA3 with the JANUS setup \\ A. Gade, C.M. Campbell, G.F. Grinyer, and D. Weisshaar \\ National Superconducting Cyclotron Laboratory, \\ Michigan State University, \\ East Lansing, 48824, MI, USA. \\ D. Cline and A.B. Hayes \\ Department of Physics and Astronomy, \\ University of Rochester, \\ Rochester, 14627, NY, USA.
}

C.Y. Wu, M. A. Stoyer and J.A. Becker

Lawrence Livermore National Laboratory, Livermore, 94550, CA, USA.

\begin{abstract}
Summary
We propose to initiate a nuclear structure program at ReA3 that utilizes low-energy nuclear reactions barrier-energy Coulomb excitation, inelastic scattering and light-ion induced transfer reactions - for nuclear structure studies of exotic nuclei provided at NSCL's reaccelerator. The science program will be enabled by the JANUS (Joint Array for NUclear Structure) setup, which will combine the detection, identification and tracking of projectile-like reaction residues in two annular double-sided $\mathrm{Si}$ strip detectors with gamma-ray detection using the Segmented Germanium Array SeGA. The emerging physics opportunities are manifold and will complement the capabilities presently provided by NSCL's fast-beam program. We present first physics cases that constitute initial measurements of broad research programs that our collaboration proposes to pursue at ReA3 and its energy upgrades.
\end{abstract}

\section{Physics Justification}

General considerations of the complementarities to existing programs at NSCL

$E \lambda$ matrix elements provide the preeminent probe of collective correlations in nuclear structure, while $M 1$ matrix elements and single-nucleon spectroscopic factors are the most direct probes of single-particle configurations. Coulomb excitation directly and selectively populates collective states with cross sections that are a direct measure of the $E \lambda$ transition strengths. Pioneering studies at the NSCL have exploited fast beams for intermediate-energy Coulomb excitation measurements of $\mathrm{B}\left(\mathrm{E} 2 ; \mathrm{O}^{+}{ }_{\mathrm{g}} \rightarrow 2^{+}{ }_{1}\right)$ strengths in light radioactive beams leading to significant advances in knowledge of the evolution of nuclear structure with isospin far from stability. Although intermediate-energy Coulomb excitation is an effective probe of the $\mathrm{B}\left(\mathrm{E} 2 ; 0^{+}{ }_{\mathrm{gs}} \rightarrow 2^{+}\right)$strengths for weak beams, the short interaction time suppresses multistep excitation processes and Coulomb-nuclear interference effects limit quantitative studies to single step [Gla98] processes.

Multistep Coulomb excitation processes become dominant for collective nuclei excited by high- $Z$ nuclei at bombarding energies close to the Coulomb barrier. This allows for the identification and spectroscopy of the yrast and yrare structures beyond the first $2^{+}$state in even-even nuclei and for particle-core coupled structures in odd- $A$ and odd-odd nuclei [Cli86, Hay07a]. For example, multiple Coulomb excitation at barrier energies populated states up to spin $40 \hbar$ in ${ }^{258} \mathrm{U}\left[\mathrm{Sim} 00\right.$ ] and $\operatorname{spin} 38 \mathrm{~h}$ in ${ }^{234} \mathrm{U}$ [Wu07]. At 
bombarding energies below the Coulomb barrier the interaction is dominated by the well-known electromagnetic force allowing for quantitative studies of nuclear structure unimpeded by uncertainties in knowledge of the interaction or reaction mechanism that plague many other spectroscopic probes in nuclear science. Sub-barrier multiple Coulomb excitation has been developed to the stage where a significant fraction of the $E 1, E 2$ and $E 3$ transition matrix elements coupling low-lying states, as well as the static E2 moments, have been measured for low-lying states up to high spin in collective nuclei [Cli86]. The much improved sensitivity of modern photon and heavy-ion detectors even allows study of collective bands built on long-lived isomeric states [Hay07b].

Low-lying quadrupole collectivity in even-even nuclei continues to provide strong indications of changes in the nuclear shell structure and allows classifying collective phenomena like rotation, vibration and critical-point symmetries. Intermediate-energy Coulomb excitation of projectile beams at energies exceeding $50 \mathrm{MeV} / \mathrm{u}$ typically excites selectively the first $2^{+}$states in even-even nuclei in a single step and thus allows for a clean determination of the $\mathrm{B}\left(\mathrm{E} 2 ; 0^{+} \rightarrow 2^{+}\right)$quadrupole excitation strength in the regime of low beam rates and thick reaction targets [Gla98,Gad08]. Cross sections measured in intermediate-energy Coulomb excitation are typically angle-integrated and sensitive to the transition matrix elements only, which do not carry information on the magnitude and sign of the static quadrupole moment and thus the nuclear shape. At low beam energies, however - driven by the significance of the adiabaticity parameter in low-energy collisions - the reorientation of the projectile spin during a collision resulting in Coulomb excitation is appreciable and leads to the excitation amplitude being proportional to the quadrupole moment including its sign in second order perturbation theory (special case of a multi-step excitation where the second step happens within the $m$-substates of the excited level). Therefore, lowenergy Coulomb excitation provides the unique opportunity to determine nuclear shapes from the measurement of the magnitude and sign of the static quadrupole moment.

Exotic odd-A nuclei have rarely been studied with intermediate-energy Coulomb excitation although the fragmentation of quadrupole strength poses stringent tests for nuclear shell-model calculations, in particular in the vicinity of developing or disappearing shell gaps. One problem is the multipolarity of the excitations, which typically has to be deduced in comparison to nuclear models, limiting the power of this approach. For barrier-energy Coulomb excitation, the shape of the differential cross section $d \sigma / d \Omega$, which can easily be measured over a substantial angular range, is sensitive to the multipolarity of the electromagnetic excitation and promises to add low-energy Coulomb excitation of odd-A nuclei to the arsenal of sensitive probes of the nuclear shell model in the regime of exotic nuclei.

For light nuclei, with $Z<10$, nuclear contributions in intermediate-energy Coulomb excitation have been found to become substantial even if the impact parameter of the collision is restricted to "safe" distances via scattering-angle cuts as typically performed [Gad08]. This means that for light, exotic nuclei, Coulomb excitation below the Coulomb barrier allows for the highest precision and eliminates the model dependency that is inherent to intermediate-energy Coulomb excitation of low- $Z$ nuclei where a modeling of the nuclear contribution - based on poorly known optical models - is mandatory.

At higher beam energies, with ReA12 or a ReA6 upgrade, inelastic proton scattering will become interesting as a complementary approach. Coulomb excitation probes only proton density distributions, whereas proton inelastic scattering is sensitive to both proton and neutron densities. Combining both measurements can thus disentangle the neutron and proton contributions to excited states. At ReA3 energies, resonant proton scattering might be of interest for experiments with a focus on nuclear astrophysics.

Our planned setup will also allow for gamma-tagged $p$ or $d$-induced single nucleon transfer reactions and thus will extend the existing transfer reaction program with beams of $>20 \mathrm{MeV} / \mathrm{u}$ towards the lowest energies. An attractive feature is the strong influence of linear and angular momentum matching on transfer cross sections. At ReA3 energies, the lowest-l orbits will be selectively populated, providing a 
very clean spectroscopy of the $s$ and $p$ states that are suspected to descend in energy and influence the nuclear shell structure as the neutron dripline is approached [Ham07]. Quasi-elastic heavy-ion induced reactions at barrier energies have been used successfully to study single-nucleon and pair-transfer cross sections in order to probe the interplay of single-nucleon spectroscopic factors or pairing correlations with collective degrees of freedom [Wu90]. For intense beams, such studies could complement $p$ or $d$ induced transfer studies where multistep coupling is absent.

The JANUS collaboration combines the NSCL group expertise in development and exploitation of intermediate-energy Coulomb excitation and transfer, with the over 4-decades of Rochester/LLNL group expertise in development and exploitation of multiple Coulomb excitation and transfer at barrier energies. The synergy of this combined expertise bodes well for the success of this planned new research initiative.

In the following we outline a few physics programs that we propose to address with beams available at NSCL's reaccelerator.

\section{Probing collectivity and shape changes in regions of shape coexistence}

The shape of the atomic nucleus is determined by the interplay of macroscopic and microscopic effects within the fermionic many-body system. For certain proton and neutron numbers, the rearrangement of a few nucleons among close-lying orbits can lead to sudden and dramatic changes of the nuclear shape and magnitude of deformation. This shape coexistence - where very different shapes or collective structures with different deformation compete in a nucleus at low excitation energy - is prominent in several regions of the nuclear chart.

The even-even self-conjugate nuclei with $28<N=Z<50$ and their neighborhood have posed a longstanding experimental challenge. The $f p$ shell with the deformation-driving $g_{9 / 2}$ orbital is large enough for these nuclei to exhibit all aspects of collective behavior including a remarkable diversity of shapes, sizable ground-state deformations, triaxiality, and the coexistence of oblate and prolate shapes at low excitation energy. These effects are generally attributed to the occurrence of well-pronounced, deformed shell gaps in the Nilsson diagram, amplified by the simultaneous occupancy of identical orbitals by valence protons and neutrons. The ground-state shape along the $N=Z$ line in the nuclear chart is supported by limited experimental information and is predicted to change from triaxial $\left({ }^{64} \mathrm{Ge}\right)$, oblate $\left({ }^{68} \mathrm{Se},{ }^{72} \mathrm{Kr}\right)$, strongly prolate $\left({ }^{76} \mathrm{Sr},{ }^{80} \mathrm{Zr}\right)$, to spherical $\left({ }^{84} \mathrm{Mo}\right)$ as $N=Z$ increases [Joh02,Sat01]. These even-even selfconjugate nuclei also are of considerable interest since the rapid-proton capture process path follows along the $\mathrm{N} \sim \mathrm{Z}$ line in this mass regime enhancing the importance of understanding the structure of these nuclei.

We propose to study this region of the nuclear chart with low-energy Coulomb excitation to (i) map the evolution of collectivity beyond the first $2^{+}$state in multiple Coulomb excitation and to (ii) determine the magnitude and sign of the static quadrupole moments of excited states to pinpoint the shape (prolate or oblate) for cases where statistics permits.

Two different methods have been pursued at SPIRAL/GANIL and ISOLDE/CERN to infer the shape of an excited state or band structure from a direct or indirect measure of the sign of the static quadrupole moment. Due to the reorientation effect in low-energy Coulomb excitation, the measured excitation cross section for a state depends on the transitional and the diagonal matrix element (which is proportional to the static quadrupole moment). This is illustrated in Figure 1 with the Coulomb excitation of ${ }^{70} \mathrm{Se}$ performed at ISOLDE. The measured cross section provides a band of possible $\mathrm{B}\left(\mathrm{E} 2 ; 2^{+} \rightarrow 0^{+}\right)$values ranging from $600 e^{2} \mathrm{fm}^{4}$ to $300 e^{2} \mathrm{fm}^{4}$ (+/- 1 sigma) - as function of the static quadrupole moment. Now, when the $\mathrm{B}(\mathrm{E} 2)$ value or life-time is already known from intermediate-energy Coulomb excitation or a life-time measurement, for example, the static quadrupole moment can be restricted as shown in Figure 1, 
where two contradicting life-time measurements point at either prolate or oblate deformation for ${ }^{70} \mathrm{Se}$. These integral measurements do not require excessive amounts of statistics and can be done at fairly low beam rates. At the energies available at ReA3, rates $\sim 10^{3}$ pps are expected to be sufficient for a typical 5day experiment and an integral measurement in this mass region.
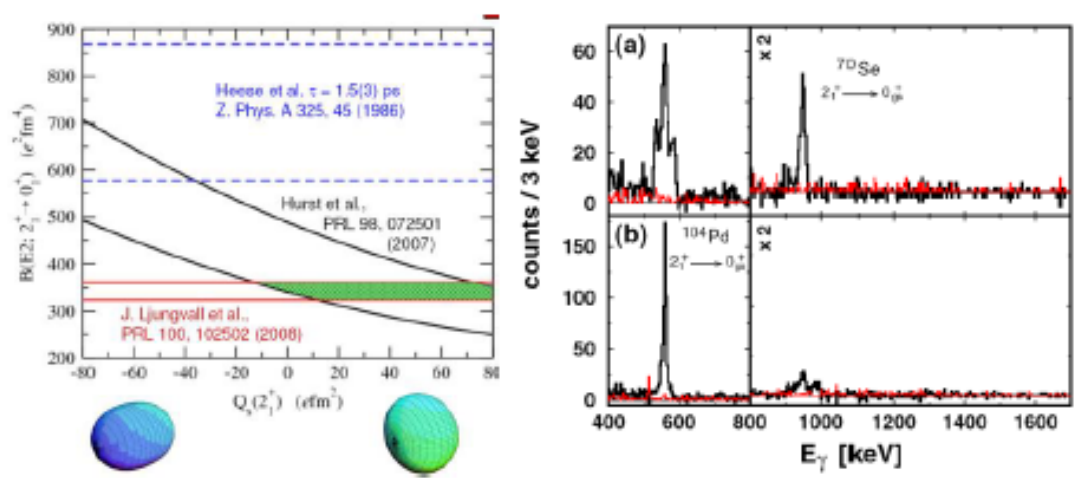

Figure 1: Determination of prolate and oblate shapes from integral low-energy Coulomb excitation experiments and life-time measurements. (Figure adapted from A. Goergen, ${ }^{70} \mathrm{Se}$ Coulomb excitation performed at ISOLDE). We note that the ${ }^{70} \mathrm{Se}$ Coulomb excitation [Hur07] was performed well below the barrier, leading to a small excitation cross section and preventing multi-step excitations. At ReA3, the experiments can be done at higher energy, leading to increased cross sections and multistep excitations.

The most direct and reliable determination of the sign is when the statistics is sufficient to measure the angular distribution of the excitation cross section with reasonable accuracy. This was the method of choice for the extensive stable-beam reorientation effect measurements performed in the 1960's [Cli69], and has been used at SPIRAL/GANIL. Figure 2 shows how the cross section distribution discriminates between prolate and oblate shapes in multiple Coulomb excitation. As shown in the spectrum, excitations up the $8^{+}$state and levels on top of excited $0^{+}$states were observed. A measurement of this quality was possible at SPIRAL/GANIL in $150 \mathrm{~h}$ of $10^{4}$ pps on a Pb target at $4.7 \mathrm{MeV} / \mathrm{u}$ [Cle07] (We note, however, that the EXOGAM full-energy peak efficiency exceeds the SeGA efficiency by about a factor of 1.4). Nuclei initially in reach are, for example, ${ }^{70} \mathrm{Se}\left[4 \times 10^{4} \mathrm{pps}\right]$ for which the result is still highly controversial (Figure 1, left), ${ }^{64} \mathrm{Ge}\left[\begin{array}{lll}3 & \mathrm{x} & 10^{4} \mathrm{pps}\end{array}\right]$ where first evidence for shape coexistence was established recently [Sta07], and ${ }^{68} \mathrm{Se}\left[1 \times 10^{4} \mathrm{pps}\right]$ for which a transitional character is predicted [Obe09].
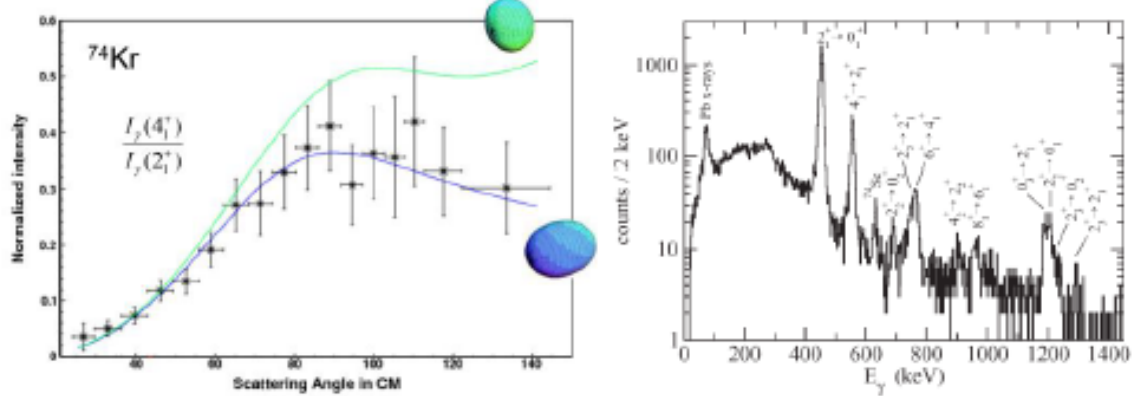

Figure 2: Left: Direct discrimination of prolate and oblate shapes from differential cross sections measured in multi-step Coulomb excitation. (Figure adapted from W. Korten, experiment performed at SPIRAL). Right: Gamma-ray spectrum detected in multi-step Coulomb excitation of ${ }^{74} \mathrm{Kr}$ at $4.7 \mathrm{MeV} / \mathrm{u}$ and $10^{4} \mathrm{pps}(150 \mathrm{~h})$ at SPIRAL/GANIL. (Figure from [Cle07]).

Other regions of the nuclear chart, where shape coexistence dominates the low-lying states, are the "Island of Inversion" around $N=20$ and neutron-rich nuclei in the vicinity of ${ }^{44} \mathrm{~S}$ around $N=28$. Here, 
intruder configurations $-\mathrm{n} p$ - $\mathrm{n} h$ excitations across the reduced $N=20$ and $N=28$ shell gaps - lead to coexisting shapes and configurations. Their study is of great importance for an understanding of the shell structure in this region as the details of the shape coexistence quantify the core-breaking and provide important benchmarks for the development of much needed cross-shell effective interactions.

For example, sulfur isotopes approaching $N=288^{44} \mathrm{~S}-{ }^{42} \mathrm{~S}\left[7 \times 10^{4} \mathrm{pps}\right]$, Argon isotopes up to ${ }^{46} \mathrm{Ar}\left[9 \times 10^{4}\right.$ pps] would be invaluable to study the competing shapes and descending intruder configurations as $N=28$ breaks down in ${ }^{42} \mathrm{Si}$.

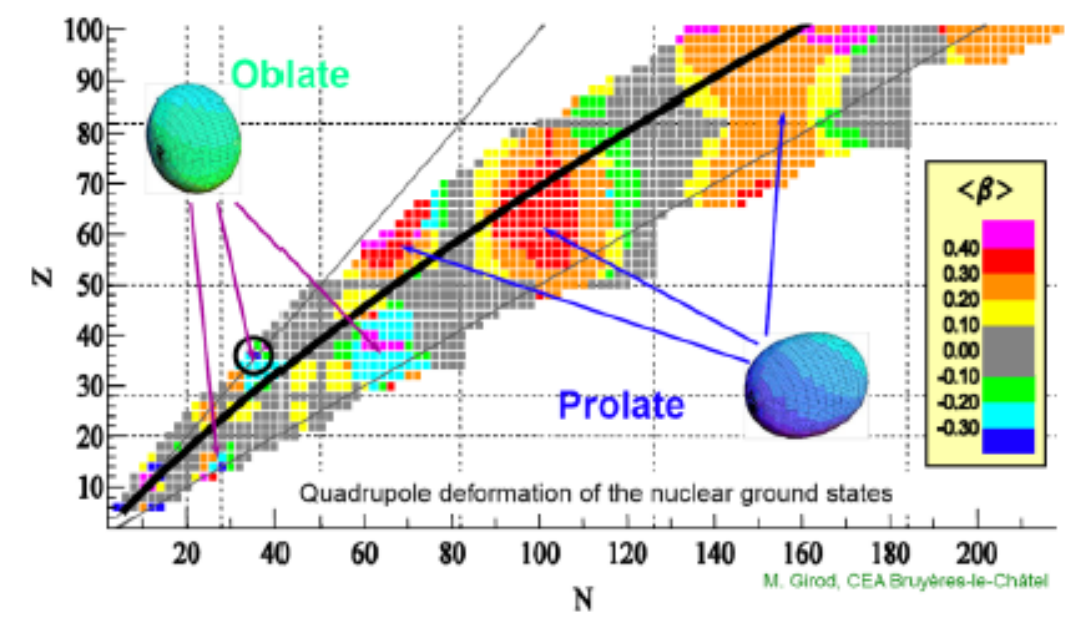

Figure 3: Ground-state shapes across the nuclear chart predicted by Hartree-Fock-Bogoliubov calculations based on the D1S Gogny force (M. Girod and S. Hilaire, CEA).

The examples specified above are a non-exclusive outline of possible first experiments. Figure 3 shows recent Hartree-Fock-Bogoliubov calculations of ground-state shapes across the nuclear chart and illustrates the rich regions where our program in the mid- and long-term future will contribute toward an understanding of the evolution of shapes and collective properties in exotic nuclei.

\section{Collectivity beyond the first excited state in the "Island of Inversion"}

Historically, measurements of the mass of the neutron-rich ${ }^{30,31} \mathrm{Na}$ isotopes revealed that they are more tightly bound than expected if one assumes a spherical shape and the validity of the conventional shell closure at neutron number $N=20$ [Thi75]. Further evidence for an onset of deformation in neutron-rich nuclei around ${ }^{31} \mathrm{Na}$ emerged from the low excitation energy of the first $2^{+}$state of ${ }^{32} \mathrm{Mg}$, and from the large $B\left(E 2 ; 0^{+} \rightarrow 2_{1}^{+}\right)$excitation strength. Shell-model calculations that allow for neutron particle-hole excitations across the $N=20$ shell gap reproduced the experimentally observed onset of deformation in this region. The $(s d)^{-2}(f p)^{+2}$ particle-hole "intruder" configurations were proposed to dominate the ground states of neutron-rich nuclei with $10 \leq Z \leq 12$ and $20 \leq N \leq 22$. This region, where the deformation-driving intruder states are energetically favored over the normal shell-model configurations, is called the "island of inversion" [War90].

The main body of evidence for deformation in this region stems from Coulomb excitation measurements of the first $2^{+}$state in even-even nuclei [Gad08]. The Tokyo Monte-Carlo Shell Model with the SDPF-M 
effective interaction has been very successful in describing the onset of low-lying quadrupole collectivity observed in experiments on even-even nuclei [Uts99]. However, intermediate-energy Coulomb excitation experiments on the odd-odd nucleus ${ }^{30} \mathrm{Na}$ [Ett08], which plays a key role in this region due to its sensitivity to the size of the $N=20$ shell gap [Uts04], revealed a significant overprediction of the collectivity by the state-of-the-art shell-model Hamiltonian as it provided no evidence for the $K=2$ rotational band predicted by shell model on top of the $2^{+}$ground state [Uts04,Ett08]. On the other hand, the recent study of low-energy Coulomb excitation of ${ }^{29} \mathrm{Na}$ shows the evidence for the neutron $s d-p f$ shell gap narrowing, which is consistent with the predictions of the Monte-Carlo Shell Model with the SDPF$\mathrm{M}$ effective interaction [Hur09]. Multiple Coulomb excitation experiments on even-even, odd-odd and odd-mass nuclei in the "island of inversion" and its vicinity would provide very stringent tests of the present effective interaction (SDPF-M) and its successors to be developed in the future. Also shape coexistence is predicted to occur all along the $N=20$ line with spherical (normal-order shell-model configurations) and strongly deformed shapes ( $f p$ intruder configurations) competing at low excitation energy where $N=20$ breaks down. Low-energy Coulomb excitation might allow disentangling the competing shapes and deformations and their evolution at the boundaries of the "island".

For the "island of inversion" around $\mathrm{N}=20$, the boundary regions approaching the "island" (i) from higher $Z-\mathrm{Si}\left({ }^{34} \mathrm{Si}\left[6 \times 10^{4} \mathrm{pps}\right],{ }^{36} \mathrm{Si}\left[2 \times 10^{4} \mathrm{pps}\right]\right)-$ and (ii) towards $\mathrm{N}=20$ along the chains of $\mathrm{Ne}$ and $\mathrm{Mg}-$ ${ }^{26} \mathrm{Ne}\left[2 \times 10^{4} \mathrm{pps}\right]$ - and $\mathrm{Mg}-{ }^{28} \mathrm{Mg}\left[10^{5} \mathrm{pps}\right]$ and ${ }^{30} \mathrm{Mg}\left[4 \times 10^{4} \mathrm{pps}\right]$ - would be important and expected to be in reach at ReA3 with sufficient intensity as well as the odd-odd and odd-mass nuclei ${ }^{28} \mathrm{Na}\left[6 \times 10^{3}\right.$ pps $],{ }^{29} \mathrm{Na}\left[2 \times 10^{3} \mathrm{pps}\right],{ }^{30} \mathrm{Na}[400 \mathrm{pps}],{ }^{27} \mathrm{Mg}\left[3 \times 10^{5} \mathrm{pps}\right]$, and ${ }^{29} \mathrm{Mg}\left[2 \times 10^{5} \mathrm{pps}\right]$.

\section{Effect of the continuum on the population of the bound excited state in ${ }^{11} \mathrm{Be}$ at low bombarding energy}

The study of electromagnetic properties of nuclei is among the most sensitive tests of the predictions of nuclear structure models. For the $a b$ initio modeling of nuclei, the electromagnetic properties in ${ }^{11} \mathrm{Be}$ provide one of the best possible cases for probing the limits of this approach. ${ }^{1} \mathrm{Be}$ is a one-neutron halo nucleus and exhibits one of the strongest known E1 transitions between bound states in a nucleus as well as a strong $E 1$ coupling to the continuum. This strength in the continuum is peaking at $\sim 800 \mathrm{keV}$ above the ground state with a long tail extending to higher excitation energy and an integrated $E 1$ strength about $1.0 e^{2} \mathrm{fm}^{2}$ [Nak94,Nun96,Pa103,Fuk04], measured by the intermediate-energy breakup reaction of ${ }^{11} \mathrm{Be}$. We propose to determine indirectly the integrated $E 1$ strength to the continuum by measuring the population of the bound excited state at $320 \mathrm{keV}$ using a ${ }^{11} \mathrm{Be}$ beam at $<25 \mathrm{MeV}$ on a ${ }^{186} \mathrm{~W}$ target. The effect of the continuum on the population of the bound excited state will be quantified by using the continuum discretized coupled channels (CDCC) method [Sak86,Sum06] for the analysis of the data. The determined $E l$ strength to the continuum will be compared to the $a b$ initio predictions, which are $\sim 80 \%$ larger than that provided by a phenomenological approach, where ${ }^{11} \mathrm{Be}$ is described as a cluster consisting of a ${ }^{10} \mathrm{Be}$ core plus one neutron and where core excitations are allowed via deformation [Nun96]. This will provide the crucial data on El strength for both, bound states and the continuum, needed for improving and testing the $a b$ initio calculations. This measurement is possible with a beam intensity of $\sim$ $10^{3}$ pps.

\section{Experimental Details}

We propose to initiate a program with ReA3 that utilizes gamma-ray tagged low-energy nuclear reactions - inelastic scattering and light-ion induced transfer reactions - for nuclear structure studies. For this, the

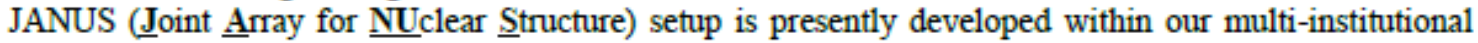
collaboration. For the detection and the angle tracking of projectile-like particles and the detection of recoiling target nuclei, three annular, double-sided Si detectors of $140 \mu \mathrm{m}$ thickness have been purchased. 
The detectors are of the type used in the BAMBINO+TIGRESS setup that is presently employed at ISAC/TRIUMF for similar low-energy scattering and reaction measurements. The front of these detectors is segmented into 32 sector strips, while the back side consists of 24 annular strips (a total of $56 \mathrm{Si}$ channels to be read out per detector). The distance to the target then determines the angular coverage. In a typical configuration with two detectors at TRIUMF, one of the $\mathrm{Si}$ detectors is mounted $3 \mathrm{~cm}$ downstream of the target, providing a range of forward laboratory angles of $20.1^{\circ}$ to $49.4^{\circ}$ [Hur09] (similar for the backward angles).

For gamma-ray detection we plan to use the SeGA array in its existing barrel configuration, usually called betaSeGA, where 16 of the 32 -fold segmented Ge detectors are arranged in a barrel around the beam pipe (Figure 4, left). We note that the segmentation of SeGA is optimal for an arrangement like this. The $\mathrm{Si}$ detectors, their mounts and the target mechanism of JANUS, will be located within the beam pipe similar to the plunger life-time setup used at NSCL in the past. The efficiency of SeGA in the envisioned barrel configuration is about $7.4 \%$ at $1 \mathrm{MeV}$. This is comparable to the efficiency of MINIBALL at ISOLDE as used for the ${ }^{70} \mathrm{Se}$ Coulomb excitation discussed above and is about $65 \%$ of the efficiency of EXOGAM at SPIRAL/GANIL.

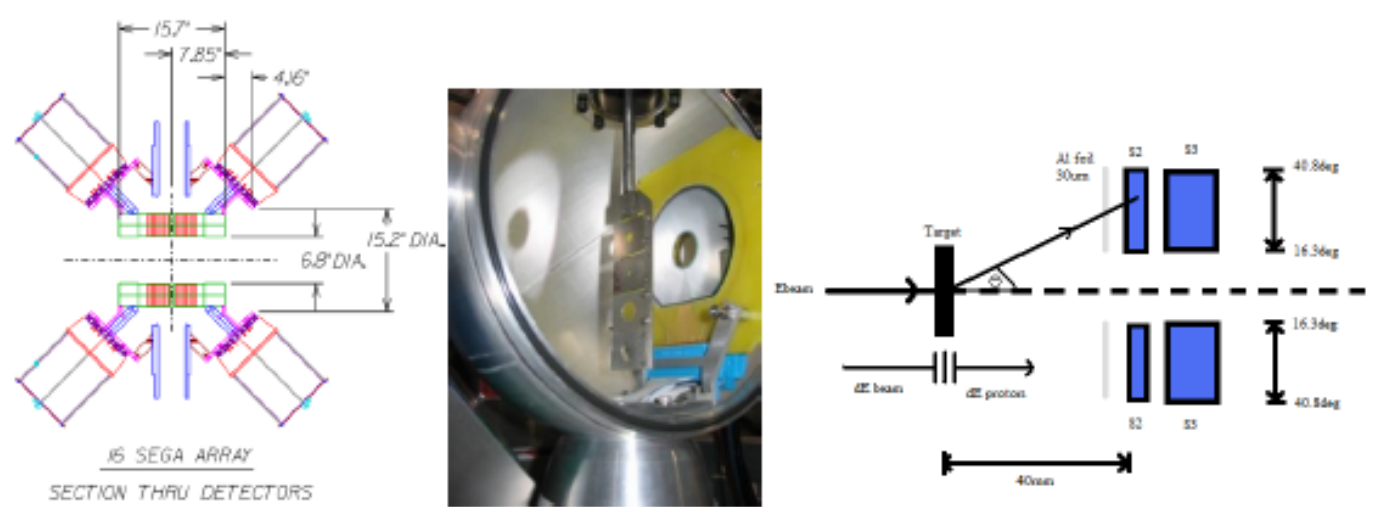

Figure 4: Left: Existing SeGA barrel configuration envisioned for the JANUS setup at ReAx. Middle: BAMBINO at TRIUMF. We note that for our SeGA configuration a particle-detector setup inside a beam pipe is optimum (similar to the NSCL plunger), while the CLOVER detector array TIGRESS at TRIUMF requires BAMBINO to have a more complicated, spherical chamber. Right: Concept of the particle detection setup which JANUS follows (Figure adapted from M. Jones, TRIUMF, 2008).

The projectile excitation cross sections will be measured relative to well-known target excitation cross sections. The semi-classical Coulomb excitation least-squares search code GOSIA [gosia] will be used for the Coulomb excitation calculations and the analysis and decomposition of transition and diagonal matrix elements. This code, originally developed at Rochester in 1980 , has been extensively updated in recent years to better match the needs of the newer detector systems plus the use world-wide for analysis of radioactive beam studies, and the Rochester group remains heavily involved in further upgrades.

\section{Educational impact}

This project involves graduate students and postdoctoral research associates at various stages in their careers and will broaden their expertise in low-energy nuclear structure experiments - from the development and the implementation of the setup to the execution of experiments at ReA3 and its envisioned future energy upgrades. We anticipate that experiments performed with the JANUS setup will result in numerous $\mathrm{PhD}$ theses in the future. 


\section{Acknowledgement:}

This work is performed partially under the auspices of the National Science Foundation grant to MSU and Rochester, and the U.S. Department of Energy by Lawrence Livermore National Laboratory under Contract DE-AC52-07NA27344.

\section{References}

[Cle07] E. Clement et al., Phys. Rev. C 75, 054313 (2007).

[Cli69] D. Cline et al., Nucl. Phys. A133, 445 (1969)

[Cli86] D. Cline, Annu. Rev. Nucl. Part. Sci. 36, 683 (1986).

[Ett08] S. Ettenauer et al., Phys. Rev. C 78, 017302 (2008).

[Fuk04] N. Fukuda et al., Phys. Rev. C 70, 054606 (2004).

[Gad08] A. Gade and T. Glasmacher, Prog. in Part. Nucl. Phys. 60, 161 (2008).

[Gla98] T. Glasmacher, Annu. Rev. Nucl. Part. Sci. 48, 1 (1998).

[gosia] GOSIA - http://www.pas.rochester.edu/ cline/Research/GOSIA.htm

[Ham07] I. Hamamoto, Phys. Rev. C 76, 054319 (2007).

[Hay07a] A.B. Hayes et al., Laser Phys. Lett. 17, 745 (2007)

[Hay07b] A.B. Hayes et al., Phys. Rev. C75 034308 (2007)

[Hur09] A.M. Hurst et al., Phys. Lett. B 674, 168 (2009).

[Joh02] A. Johnson et al., J. Phys. A 13, 9 (2002) and references within.

[Nun96] F.M. Nunes, I.J. Thompson, and R.C. Johnson, Nucl. Phys. A 596, 171 (1996).

[Nak94] T. Nakamura et al., Phys. Lett. B 331, 296 1994).

[Obe09] A. Obertelli et al., Phys. Rev. C 80, 031304(R) (2009).

[Pa103] R. Palit et al., Phys. Rev. C 68, 034318 (2003).

[Sak86] Y. Sakuragi, M.Yahiro, and M. Kamimura, Prog. Theor. Phys. Suppl. 89, 136 (1986).

[Sat01] W. Satula et al., Phys. Rev. Lett. 87, 052504 (2001).

[Sim00] M.Simon et al., Unpublished

[Sta07] K. Starosta et al., Phys. Rev. Lett. 99, 042503 (2007).

[Sum06] N.C. Summers et al., Phys. Lett. B 650, 124 (2007).

[Thi75] C. Thibault et al., Phys. Rev. C 12, 644 (1975).

[Uts99] Y. Utsuno et al., Phys. Rev. C 60, 054315 (1999).

[Uts04] Y. Utsuno et al., Phys. Rev. C 70, 044307 (2004).

[War90] E. K. Warburton et al., Phys. Rev. C 41, 1147 (1990).

[Wu90] C.Y. Wu et al., Annu. Rev. Nucl. Part. Sci. 40, 285 (1990).

[Wu07] C.Y. Wu et al., ANL ATLAS proposal, Sept. 2007. 
MICHICAN STATE

U N I V E R S I T Y

January 29,2010

Dr. Alexandra Gade

NSCL

Michigan State University

East Lansing, MI 48824-1321

Dear Alexandra:

The Program Advisory Committee (PAC 34) of the National Superconducting Cyclotron Laboratory met on January 19-20, 2010 to consider proposals for beam time at the Coupled Cyclotron Facility and letters of intent for experiments at the upcoming reaccelerated beam facility. Your proposal, Low-Energy Coulomb Excitation, Inelastic Scattering and Transfer Reaction Measurements at ReA3 with the JANUS Setup (No. 09058), was included in this review.

The Committee viewed your letter of intent favorably. They felt that the proposed JANUS facility promises to become a standard setup for a wide range of experiments at ReA3. The Committee was pleased to see example experiments listed that would be difficult to do at other facilities, and view these as priorities for future proposals. They noted that one of the compelling aspects of this LOI was that it will enable a wide range of interesting measurements at beam intensities in the $10^{3}$ to $10^{4} \mathrm{pps}$ range. It could therefore likely be able to take advantage of ReA3 early on. We look forward to future proposals along this line using ReA3.

Please notify your colleagues on the letter of intent of this decision. Thank you for your interest in performing experiments at the reaccelerated beam facility.

NSCL

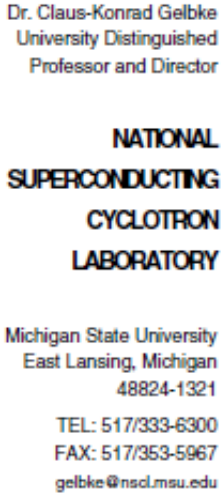

The Mchigan State Uhiversity IDEA is institutional Diversity Excelence in Action

MSU is an affirmative-action equal-opponturity insthution.
Sincerely,

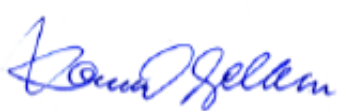

C. Konrad Gelbke

Director 